\title{
Studies of the radial gradient of rotation near the solar surface
}

\author{
Dissertation \\ zur Erlangung des mathematisch-naturwissenschaftlichen Doktorgrades \\ "Doctor rerum naturalium" \\ der Georg-August-Universität Göttingen \\ im Promotionsprogramm PROPHYS \\ der Georg-August University School of Science (GAUSS)
}

vorgelegt von

Atefeh Barekat

aus Teheran, Iran

Göttingen, 2018 
Betreuungsausschuss

Prof. Dr. Laurent Gizon

Max-Planck-Institut für Sonnensystemforschung und

Institut für Astrophysik, Georg-August-Universität Göttingen, Deutschland

Prof. Dr. Stefan Dreizler

Institut für Astrophysik, Georg-August-Universität Göttingen, Deutschland

Dr. Jesper Schou

Max-Planck-Institut für Sonnensystemforschung, Göttingen, Deutschland

Dr. Aaron Birch

Max-Planck-Institut für Sonnensystemforschung, Göttingen, Deutschland

Mitglieder der Prüfungskommision

Referent: Prof. Dr. Laurent Gizon

Max-Planck-Institut für Sonnensystemforschung und

Institut für Astrophysik, Georg-August-Universität Göttingen, Deutschland

Korreferentin: PD Dr. Olga Shishkina

Max-Planck-Institut für Dynamic und Selbstorganisation, Göttingen, Deutschland

Weitere Mitglieder der Prüfungskommission:

Prof. Dr. Ulrich Christensen

Max-Planck-Institut für Sonnensystemforschung, Göttingen, Deutschland

Prof. Dr. Stefan Dreizler

Institut für Astrophysik, Georg-August-Universität Göttingen, Deutschland

Prof. Dr. Ariane Frey

Physikalisches Institut, Georg-August-Universität Göttingen, Deutschland

Prof. Dr. Ansgar Reiners

Institut für Astrophysik, Georg-August-Universität Göttingen, Deutschland 


\section{Contents}

Summary 5

\begin{tabular}{lll}
\hline 1 & Introduction & 7
\end{tabular}

1.1 The $\operatorname{Sun} \ldots \ldots \ldots \ldots \ldots \ldots \ldots$

1.2 Helioseismology . . . . . . . . . . . . . . . . . . . . 9

1.2 .1 Observational data . . . . . . . . . . . . . . . 10

1.2 .2 Measuring internal rotation . . . . . . . . . . . . . . . 12

1.3 Solar differential rotation $\ldots \ldots \ldots \ldots$. . . . . . . . . . 15

1.3 .1 Surface rotation . . . . . . . . . . . . . . . . . . . . . . . . . . . .

1.3 .2 Internal rotation . . . . . . . . . . . . . . . . . . . 18

1.4 The near-surface shear layer (NSSL) . . . . . . . . . . . . . . . . . . . 19

1.5 Time variation of the differential rotation . . . . . . . . . . . . . 20

1.6 Thesis motivation $\ldots \ldots \ldots \ldots \ldots . \ldots \ldots$

2 Paper I: The radial gradient of the near-surface shear layer of the Sun 25

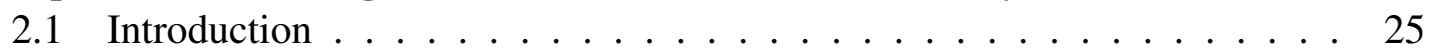

2.2 Observations . . . . . . . . . . . . . . . . 26

2.3 Analysis of $\mathrm{f}$ mode data . . . . . . . . . . . . . . . . . . . . . . 27

2.4 Results . . . . . . . . . . . . . . . . . . . . . . . . . . . . 29

2.5 Conclusion . . . . . . . . . . . . . . . . . 32

3 Paper II: Solar-cycle variation of the rotational shear near the solar surface 35

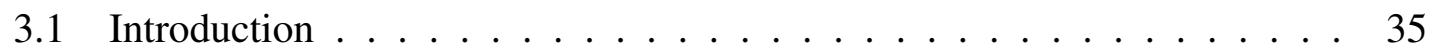

3.2 Observational data $\ldots \ldots \ldots \ldots \ldots \ldots \ldots$

3.3 Method . . . . . . . . . . . . . . . . . . . . . . . 38

3.4 Results . . . . . . . . . . . . . . . . . . . . . . . . 38

3.4 .1 Results obtained from 72-day vs. 360-day data . . . . . . . . . . . . 39

3.4.2 Solar cycle variation of the radial gradient . . . . . . . . . . . . . . . . . . . . . . . . . . 40

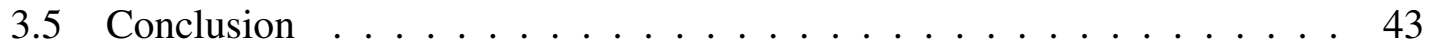

\begin{tabular}{|lll}
\hline 4 & Discussion and outlook & 47
\end{tabular}

4.1 Comparison with various other data sets . . . . . . . . . . . . . . . . 48

$4.1 .1 \quad$ Radial gradient obtained from full-disk MDI data . . . . . . . . . 48

$4.1 .2 \quad$ Radial gradient obtained from Medium- $l$ HMI data . . . . . . . . 51

4.2 Theoretical implications . . . . . . . . . . . . 52

4.3 Outlook . . . . . . . . . . . . . . . . . . . . 53 
\begin{tabular}{ll}
\hline Bibliography & 55
\end{tabular}

\begin{tabular}{ll}
\hline Publications & 61
\end{tabular}

\begin{tabular}{ll}
\hline Acknowledgments & 63
\end{tabular} 


\section{Summary}

Continuous observations of solar oscillations over the last twenty years have provided an opportunity to investigate interior properties of the Sun and their variations over the last two solar cycles. In this thesis, I focus on measuring the radial gradient of the rotation rate close to the surface of the Sun and its variations during solar cycle 23 and 24. For this study, I used for the first time f mode frequency splitting data obtained from 360-day time series of Medium- $l$ data from both the Michelson Doppler Imager (MDI: 1996-2010) on board the Solar and Heliospheric Observatory and the Helioseismic and Magnetic Imager (HMI: 2010-2015) on board the Solar Dynamics Observatory to study the near-surface shear layer. Furthermore, I used reprocessed Medium- $l$ MDI and HMI data obtained from 72-day time series that contain less systematic errors than those used in previous studies.

In contrast to previous studies, I found that the logarithmic radial gradient of the rotation rate does not change sign at $55^{\circ}$ latitude. Rather, I found that the value of the radial gradient stays negative and varies between approximately -1 and -0.9 from the equator up to about $50^{\circ}$ latitude. Above $50^{\circ}$ latitude the measurements were found to be unreliable based on the discrepancies between the results obtained from different data sets. These include differences between the results obtained from modes in common between 72-day and 360-day time series from each instrument separately. Moreover, the mentioned comparison of HMI data showed that the data obtained from 72-day time series are likely the ones that suffer from systematic errors that are currently not understood. Furthermore, I measured the radial gradient obtained from recently released MDI full-disk data which have been processed differently than Medium- $l$ data. The results showed that the values of the radial gradient at low latitudes are the same as previous measurements to within $1 \sigma$. These measurements are in agreement with those predicted by the Kitchatinov and Rüdiger (2005) differential rotation model.

By comparing the gradient relative to its nineteen year (1996-2015) time averaged value, I found that it varies by about $\pm 10 \%$ and closely follows the solar activity. The change in the radial gradient with solar cycle may provide a means of measuring the subsurface magnetic field as suggested by Kitchatinov (2016). 



\section{Zusammenfassung}

Kontinuierliche Beobachtungen von Oszillationen in der Sonne über in den letzten 20 Jahre ermöglicht, die innere Struktur der Sonne und ihre Veränderungen während der letzten beiden Sonnenzyklen zu erforschen. In dieser Doktorarbeit bestimme ich den radialen Gradienten der Sonnenrotation nahe der Sonnenoberfläche sowie seine Variationen innerhalb der Sonnenzyklen 23 und 24. Für diese Studie verwende ich zum ersten Mal Messungen der Frequenzaufspaltung der f Mode, die aus Zeitreihen mit einer Länge von 360 Tagen gewonnen wurden, um die oberflächennahe Scherungsschicht zu erforschen. Die Daten stammen sowohl von Michelson Doppler Imager (MDI: 1996 - 2010) an Bord des Solar and Heliospheric Obervatory als auch vom Helioseismic and Magnetic Imager (HMI) an Bord des Solar Dynamics Observatory. Außerdem verwende ich Zeitreihen mit einer Länge von 72 Tagen, die aus dem Medium- $l$-Programm von MDI und von HMI stammen. Diese wurden mit neuen Methoden erstellt, sodass sie weniger systematische Fehler enthalten als die Daten, die in vorherigen Arbeiten verwendet wurden.

Im Gegensatz zu vorherigen Studien kann ich keinen Vorzeichenwechsel des logarithmischen radialen Gradienten der Sonnenrotation bei einem Breitengrad von $55^{\circ}$ feststellen. Stattdessen bleibt das Vorzeichen negativ und der Wert des Gradienten schwankt zwischen ungefähr -1 am Äquator und -0.9 auf hören Breiten. Oberhalb des 50. Breitengrad sind die Daten unzuverlässig, da es Diskrepanzen zwischen den Ergebnissen gibt, die mit den verschiedenen Datensätzen erzielt wurden. Sowohl bei MDI als auch bei HMI liefern die Zeitreihen mit einer Länge von 72 Tagen und die mit einer Länge von 360 Tagen unterschiedliche Ergebnisse, selbst wenn nur die Moden verwendet werden, die in beiden Datensätze vorkommen. Zusätzlich hat der Vergleich der HMI Daten gezeigt, dass die 72 Tage langen Zeitreihen von HMI vermutlich einen systematischen Fehler aufweisen, der bis jetzt nicht verstanden ist. Außerdem habe ich den radialen Gradienten aus kürzlich veröffentlichten MDI Daten, die die gesamte Sonnenscheibe zugrunde legen und die anders als die Medium- $l$-Daten erstellt wurden, bestimmt. Die Ergebnisse haben gezeigt, dass die Werte des radialen Gradienten bei niedrigen Breitengraden mit einer Genauigkeit von $1 \sigma$ mit vorherigen Messungen übereinstimmen. Diese Messungen sind im Einklang mit Vorhersagen des theoretischen Modells der differentiellen Rotation der Sonne von Kitchatinov and Rüdiger (2005).

Indem ich den Gradienten mit seinem zeitlichen Mittel über 19 Jahre (1996 - 2015) verglichen habe, konnte ich feststellen, dass er um etwa $\pm 10 \%$ schwankt und so eng der Variation der Sonnenaktivität folgt. Diese Schwankungen des radialen Gradienten im Einklang mit der Sonnenaktivität könnten laut Kitchatinov (2016) die Möglichkeit bieten, die Stärke des solaren Magnetfeldes unterhalb der Sonnenoberfläche zu bestimmen. 



\section{Introduction}

In the last few decades, unprecedented information about the interior structure and rotation of the Sun has become available. Additionally, about two decades of continuous observations of the Sun provide an excellent opportunity of monitoring the temporal variation of the interior structure of the Sun and its relationship to the $\sim 11$ year sunspot cycle. Several studies have investigated the temporal variation of the solar rotation rate at different depths and latitudes. However, only few studies attempted to measure the radial and latitudinal gradient of the interior rotation rate of the Sun. In this thesis, I focus on measuring the radial gradient close to the surface and its variation in relation to the solar cycle as the quantitative measurements of this gradient can provide useful information for both dynamo and differential rotation theories.

In this section, I provide some basic information about the Sun. Then, I introduce the means of measuring the rotation rate and the observational data that I use in this thesis. I explain what we have learned about the solar surface rotation in the last few hundred years and its interior rotation in the last few decades. In particular, I focus on the solar rotation rate and its gradient close to the surface. Finally, I present the motivation of the thesis at the end of this chapter.

In the rest of the thesis, I start by presenting my published article on measuring the radial gradient of the near-surface shear layer of the Sun in Section 2 and my published article about its temporal variations in Section 3. I finish this thesis by providing the discussion and outlook in Section 4

\subsection{The Sun}

The Sun is a rotating ball of hot gas with a radius $\left(R_{\odot}\right)$ of roughly $700 \mathrm{Mm}$. It radiates thermal energy continuously into space from its surface. This energy is provided by nuclear reactions taking place in the core where hydrogen is burned into helium. Then, the energy is transported from the core to the surface of the Sun mainly by radiation and convection. These two means of energy transport from the core define two different zones inside the Sun. In the radiative zone, which is located between approximately $0.3 R_{\odot}$ and $0.7 R_{\odot}$, the energy transport is dominated by radiation. In the convection zone, which is located between approximately $0.7 R_{\odot}$ and the surface, the energy transport is dominated by convection which drives motions on broad range of scales. The interior of the Sun is shown schematically in Fig. 1.1 .

The surface of the Sun shows a cyclic activity with a period of about 11 years. Sunspots are the most visible manifestations of this cycle (Schwabe 1844). They appear as dark spots which emerge at the surface and then decay with a lifetime of few days up to about a 


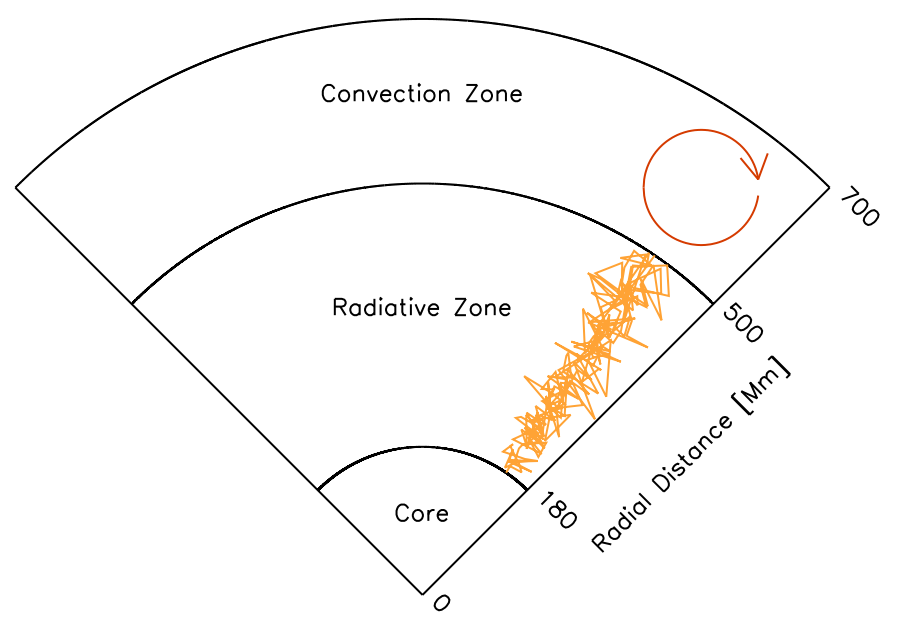

Figure 1.1: Sketch of the interior structure of the Sun: the core, the radiative zone and the convection zone. The means of the energy transport are also shown schematically in the radiative and convection zone.

month. Hale (1908) found that these spots contain concentrated magnetic field. It turns out that the polarity of the global magnetic field reverses its sign every 11 years which determines the magnetic cycle of 22 years.

The solar cycle starts by emergence of sunspots at about $\pm 30^{\circ}$ latitudes. The latitude of the emergence moves towards the equator as the cycle progresses which is known as "equatorward migration" of the activity belt. This behavior is nicely seen in the so-called

\section{DAILY SUNSPOT AREA AVERAGED OVER INDIVIDUAL SOLAR ROTATIONS}

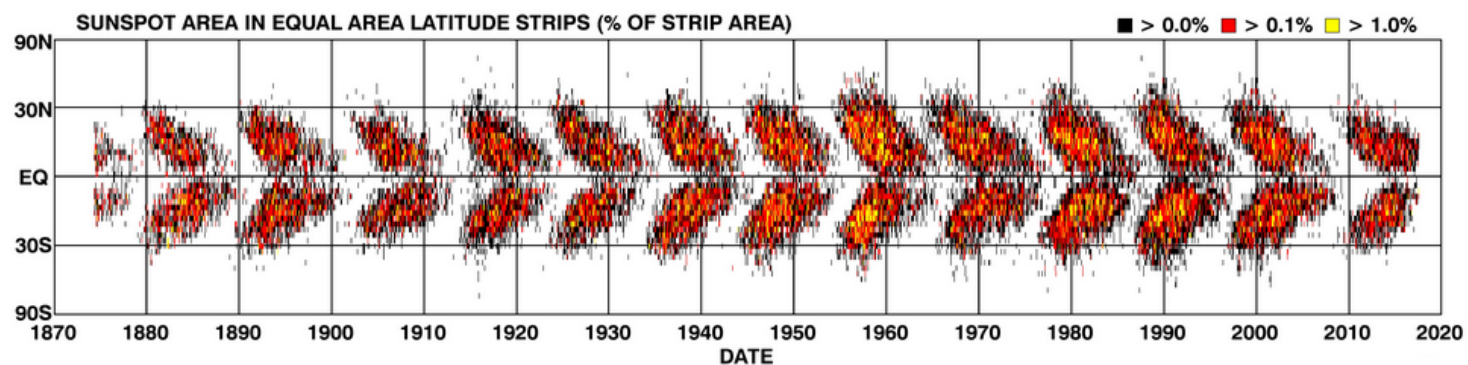

Figure 1.2: Temporal evolution of sunspots area at different latitudes observed at the surface of the Sun. The letter N and S in the y-axis stands for North and South hemisphere, respectively. Courtesy of D. Hathaway, taken from http://solarscience.msfc.nasa. gov/greenwch.shtml. 
"butterfly diagram", an example of which is shown in Fig. 1.2. As the cycle evolves the number of spots increases until it reaches to its maximum value. This stage of the solar cycle is referred to as "solar maximum". Then the number of spots decreases towards the end of the cycle and reaches a minimum value, at the "solar minimum". Although we have a record of sunspot observations for a few hundred years, the mechanism behind the cyclic behavior is not well understood. However, it is believed that the cyclic magnetic field is generated by a dynamo operating below the surface of the Sun (Brandenburg and Subramanian 2005, Charbonneau 2010) driven by differential rotation and turbulent convection.

Later in the 20th century, obtaining information about the physical properties of the Sun below its surface became possible. With drastic improvements in the observational techniques and the striking discovery of the oscillations of the Sun we could obtain unprecedented information about the interior of the Sun. The technique that provides such information is known as "helioseismology".

\subsection{Helioseismology}

Accessing detailed information about the interior of the Sun became possible in the 1970s by discovering that the Sun oscillates in millions of different modes. The discovery began by an observational attempt at estimating the life time and other properties of the convective cells which cover the whole surface of the Sun known as granulation. For this purpose Leighton et al. (1962) used Doppler velocity data and noticed fluctuations in Doppler velocity over time which lead to the discovery of five minute oscillations of the Sun. Ulrich (1970) explained these oscillations by proposing the idea that the acoustic waves are trapped in resonant cavities inside the Sun. Using his model, he calculated the dispersion relation of these trapped waves. The dispersion relation describes how the frequency $v$ of a wave is related to its wavenumber $k=2 \pi / \lambda$, where $\lambda$ is the wavelength of the wave. He showed that there exist several discrete ridges in the $k$ - $\omega$ diagram (diagnostic diagram) of these waves, where $\omega=2 \pi v$ is the angular frequency. He also noted that these ridges were not observed because of the poor temporal and spatial resolution of the observations.

After several improvements in observational techniques, Deubner (1975) followed the advice of Ulrich and made an observation with higher temporal and spatial resolution. The power spectrum of his observation matched well with the theory of Ulrich (1970). With this confirmation a new discipline of solar physics was born which is known as "helioseismology". Nowadays, measuring the frequencies of these modes provides information about the dynamics and structure of the interior of the Sun.

The oscillations of the Sun can be described in terms of normal modes. The eigenfunctions of these normal modes can be separated into radial and horizontal components, see for details (Aerts et al.2010). The frequency $v_{n l m}$ of these normal modes is described by three integer numbers $n, l$ and $m$. The radial order $n$ is the number of nodes of the radial component of the eigenfunction. Spherical harmonic degree $l$ is the number of nodal lines at the surface. The azimuthal order $m$ is the number of nodal lines crossing the equator. For a fixed value of $l$ there exist $2 l+1$ values of $m$, with $-l \leqslant m \leqslant l$. The modes with radial order $n>0$ are known as p modes. The restoring force for these modes is pressure.

The modes with $n=0$ are known as f modes. These modes are known as surface 


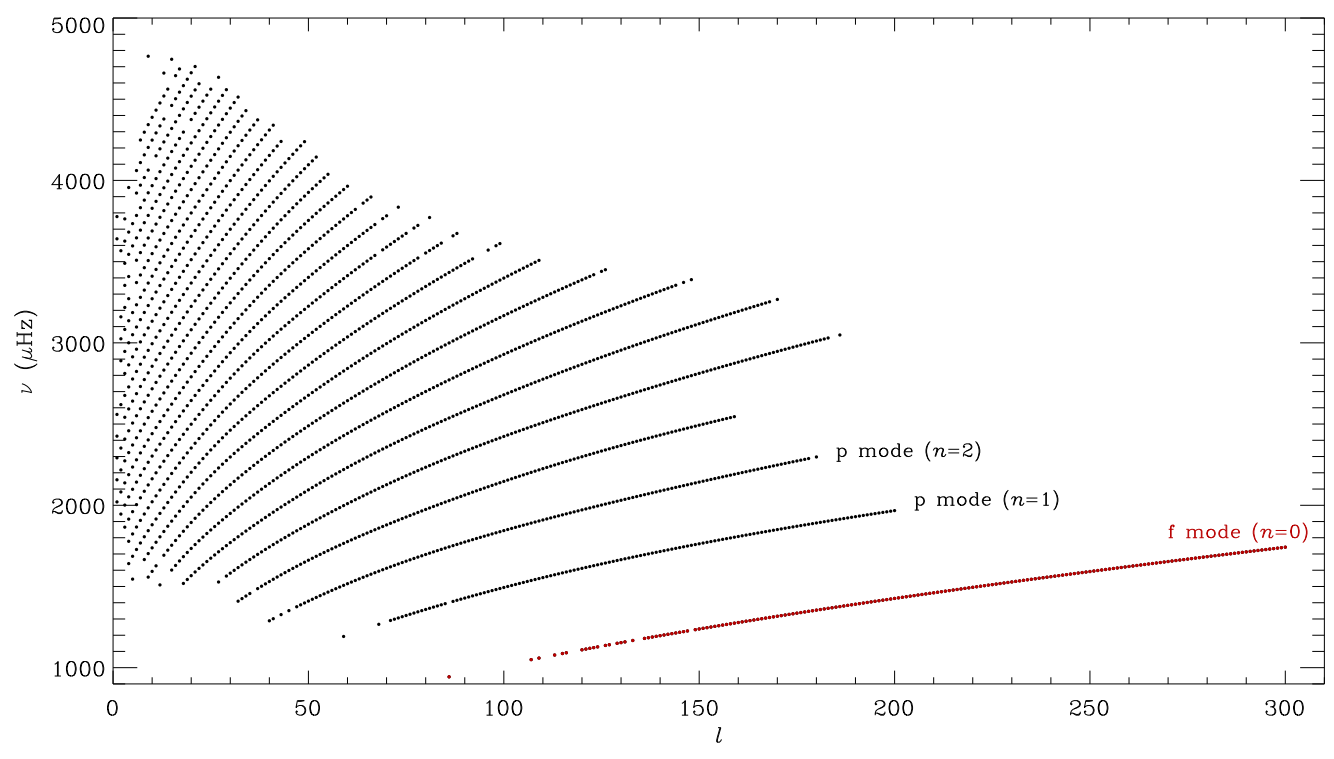

Figure 1.3: Frequency versus angular degree $l$ of solar oscillations obtained from 360-day time series of MDI data starting 10 April 2000. Each ridge corresponds to a different value of the radial order $n$. The lowest ridge shows the f mode (red) and the other ridges show $\mathrm{p}$ modes (black) where only the first two ones are labeled.

gravity modes as they have a dispersion relation and eigenfunctions similar to the waves that form on the surface of an ocean (Gough 1993). In the case of a spherically symmetric star the frequencies of these modes follow an approximated dispersion relation

$$
\omega_{l}^{2} \approx g k_{h}
$$

where $k_{h}=L / R_{\odot}$ is the horizontal spatial wavenumber, $L=\sqrt{(l(l+1))}$ and $g=274 \mathrm{~m} / \mathrm{s}^{2}$ is the gravitational acceleration at the surface of the Sun (Stix 2004). It is clear from Eq. (1.1) that $\mathrm{f}$ mode frequencies depend only on the gravity and the angular degree of the mode. An example of a diagnostic diagram of solar oscillation obtained from one of the data sets used in this thesis is shown in Fig. 1.3. As shown in this figure, f modes have lower frequencies than $\mathrm{p}$ modes. For a fixed frequency, f modes can probe shallower layers than $\mathrm{p}$ modes.

In this thesis I only consider f modes as they probe the layers that I want to study. The observational data used in this thesis study are provided from two instruments that I will introduce in the next section.

\subsubsection{Observational data}

The frequencies of oscillation of the Sun are typically measured from two main types of data: intensity and Doppler shift. An example of a Doppler image of the Sun is shown in Fig. 1.4 In this figure, the Doppler shift due to the rotation of the Sun dominates. The left side of the image is shifted to lower values (moving toward us) and the right side to higher velocities (moving away from us). The procedure used for obtaining the mode parameters from raw Doppler images is explained in Larson and Schou (2015). 


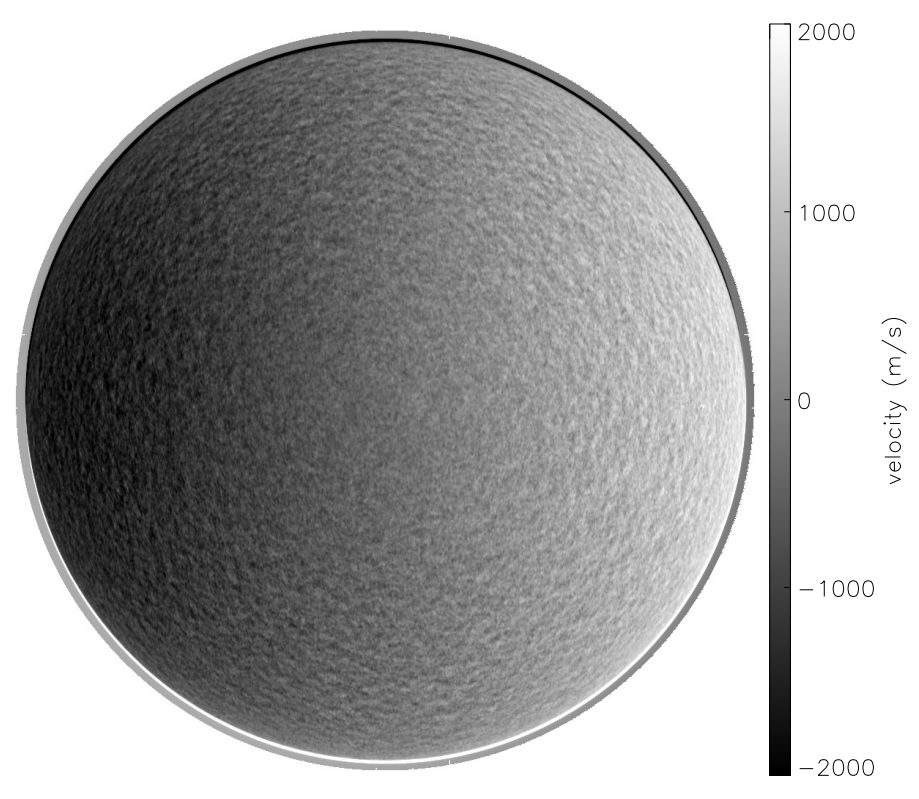

Figure 1.4: A single MDI Doppler image of the solar disk from 16 May 1996.

There exist several space and ground based instruments that provide these data sets. Here, I only introduce the two instruments which provided the data used in this thesis. The first one is the Michelson Doppler Imager (MDI; Scherrer et al. 1995) on board of the Solar and Heliospheric Observatory (SOHO) shown in left panel of Fig. 1.5. The second one is the Helioseismic and Magnetic Imager (HMI; Schou et al.2012) on board of the Solar and Dynamics Observatory (SDO) shown in right panel of Fig. 1.5 .

SOHO is located between the Earth and the Sun at a distance of $1.5 \times 10^{6} \mathrm{~km}$ from the Earth and orbits around the first Lagrangian point. MDI provided continuous Doppler images from May 1996 to April 2011 using a $1024 \times 1024$ CCD camera. The images were taken using the $6768 \AA \mathrm{Ni}$ I photospheric absorption line generally with a cadence of 60 seconds. There were only two long interruptions in the data which occurred in 1998 and lasted for $\sim 108$ and $\sim 36$ days.

SDO is in geosynchronous orbit around the Earth with a semi-major axis of about $42 \times 10^{3} \mathrm{~km}$. HMI provides continuous Doppler images using a $4096 \times 4096$ CCD camera with a cadence of 45 seconds. HMI uses the $6173 \AA$ Fe I absorption line and has been operating since May 2010. The operation of the MDI instrument was halted after one year of successful operation of HMI. Therefore, there is only a one year overlap (30 April 2010 to 24 April 2011) between the data sets of the two instruments. The spacecraft and the time span that are used in this thesis are shown in Fig. 1.5

The MDI instrument only provided full disk Doppler images for short periods of time each year because of telemetry limitations. Therefore, in order to use continuous data, I consider $a$-coefficients obtained from the Medium- $l$ program data (Scherrer et al. 1995, Kosovichev et al. 1997). The Medium- $l$ data are obtained from Doppler images that have been convolved with a Gaussian kernel, sub-sampled by a factor of 5 and cropped to $0.9 R_{\odot}$ of full disk image. I note that the three mentioned steps were done on board. The maximum value of the angular degree covered by the Medium- $l$ program data is roughly 

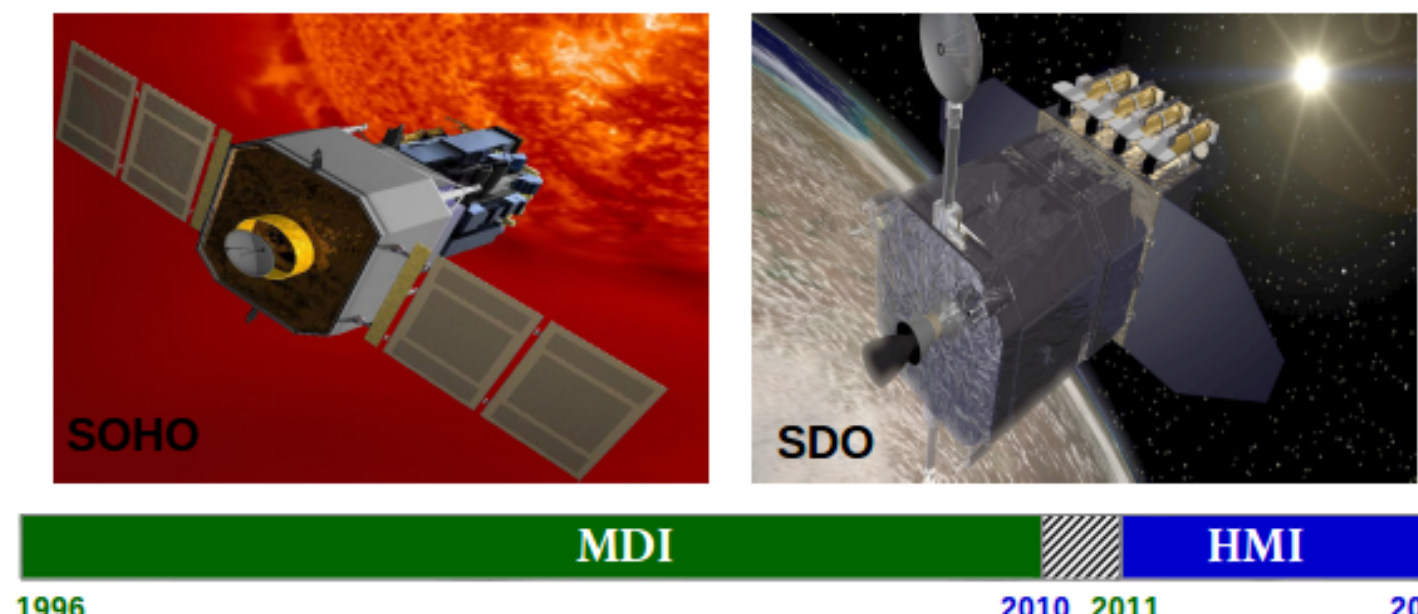

1996

20102011

HMI

2015

Figure 1.5: Time span of data used in this thesis. SOHO spacecraft is shown in the left panel (Image courtesy of NASA/Lutkus/H.Zell) and SDO spacecraft is shown in the right panel (Image courtesy of NASA/Goddard Space Flight Center/Conceptual Image Lab). The green color represents the time span of MDI data and the blue HMI that of data. The striped lines show the time span where there is overlap between MDI and HMI data.

$l=300$. Fortunately, HMI does not have telemetry limitations and the mode frequencies can be obtained from full disk images. This means that the MDI data has poorer resolution by a factor of 20 than the HMI data. We note here that the resolution of MDI should not affect the global mode parameter (Larson and Schou 2018).

Although there are several differences between these two instruments, which lead to some small differences in the beginning of the processing of the data, the method used for obtaining the $a$-coefficients is basically the same.

\subsubsection{Measuring internal rotation}

Solar oscillations are influenced by rotation. The waves that propagate along or against the direction of rotation are shifted to larger or smaller frequencies, respectively.

This effect can also be explained in terms of normal modes. In a non-rotating star, mode frequencies $v_{n l m}$ of a multiplet $(n, l)$ are degenerate in $m$. Rotation breaks this degeneracy, and splits the multiplet into $2 l+1$ distinct frequencies. This frequency splitting can be seen in the $m-v$ power spectrum of solar oscillations obtained from Doppler images. An example of such a power spectrum is shown in Fig. 1.6 for $l=100$. Each band in this figure corresponds to a different value of $n$. The ridges in such a power spectrum are S-shaped. This shape indicates that the Sun rotates faster at the equator than at the poles as the slope at higher values of $|m|$ is steeper than at the lower ones. I note that the modes with $m= \pm l$ are concentrated at the equator and as the absolute value of $m$ decreases toward zero, the modes get extended toward higher latitudes.

We would expect to see a single stripe for each band, but there are several. This is because we can only observe less than half of the Sun, which makes it impossible to isolate modes perfectly (Schou and Brown 1994). As a consequence, different neighboring modes 


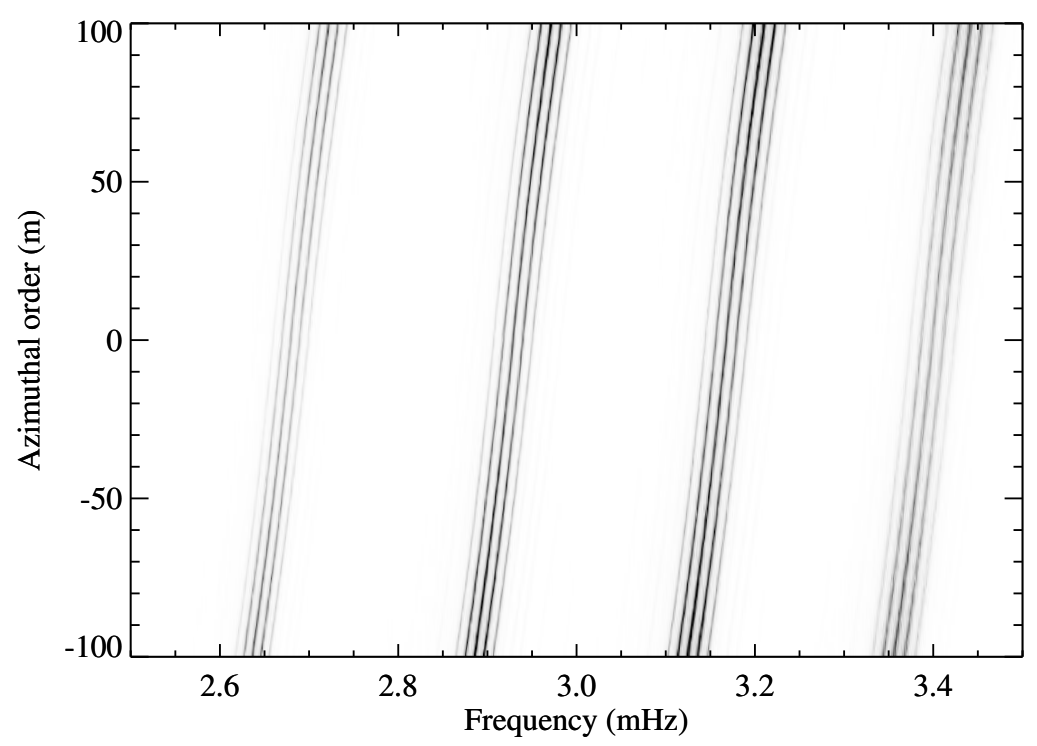

Figure 1.6: $m-v$ power spectrum of $l=100$ obtained from a 360-day HMI time series starting 30 April 2010. The values of $n$ are 5, 6, 7 and 8 from left to right for each multiple ridge structure, respectively (Courtesy of J. Schou).

leak into a given mode, which then causes a multiple ridge structure.

Instead of obtaining individual mode frequencies with a certain value of $n l m$, it is more common to express each mode frequency as a mean multiplet frequency $v_{n l}$ plus a polynomial expansion that takes into account the $m$-dependency

$$
v_{n l m}=v_{n l}+\sum_{j=1}^{j_{\max }} a_{n l, j} \mathcal{P}_{j}^{(l)}(m),
$$

where $\mathcal{P}_{j}^{(l)}$ are orthogonal polynomials of degree $j$ and $\left.a_{n l, j}\right]^{1}$ are the so-called $a$-coefficients (Schou et al. 1994).

The rotational splitting can be obtained analytically (Lynden-Bell and Ostriker 1967. Cox 1980) by taking into account the Coriolis force and the advection in the inertial frame of the Sun. The perturbation in the angular frequency $\delta \omega_{n l m}$ caused by rotation is given by

$$
\delta \omega_{n l m}=\int_{0}^{1} \int_{0}^{\pi} K_{n l m}(r, \theta) \Omega(r, \theta) r \mathrm{~d} \theta \mathrm{d} r
$$

where $\theta$ is colatitude and $r$ is fractional radius. $K_{n l m}$ are kernels that determine the contribution of the rotation rate at different depths and latitudes to the rotational splittings. The kernels depend on the mode eigenfunctions and density and are given by (Schou et al.

1 The comma in the $a$-coefficient is used for separation and not to indicate a derivative. 
1994)

$$
\begin{aligned}
K_{n l m}(r, \theta)=\frac{m}{I_{n l}}\left\{\xi _ { n l } ( r ) \left[\xi_{n l}(r)-\frac{2}{L}\right.\right. & \left.\eta_{n l}(r)\right] P_{l}^{m}(u)^{2}+\frac{\eta_{n l}^{2}(r)}{L^{2}}\left[\left(\frac{d P_{l}^{m}(u)}{d u}\right)^{2}\left(1-u^{2}\right)\right. \\
& \left.\left.-2 P_{l}^{m}(u) \frac{d P_{l}^{m}(u)}{d u} u+\frac{m^{2}}{1-u^{2}} P_{l}^{m}(u)^{2}\right]\right\} \rho(r) r \sin \theta
\end{aligned}
$$

where

$$
I_{n l}=\int_{0}^{1}\left[\xi_{n l}^{2}(r)+\eta_{n l}^{2}(r)\right] \rho(r) r^{2} \mathrm{~d} r
$$

$P_{l}^{m}$ is an associated Legendre polynomial, $u=\cos \theta$, and $\rho$ is density. $\xi_{n l}$ and $\eta_{n l}$ are the radial and horizontal displacements of the mode eigenfunctions, respectively. The kernels are only sensitive to the north-south symmetric component of the rotation rate as they are even functions of $u$. This means that we cannot distinguish between the rotation rate in the two hemispheres using global oscillation mode frequencies. However, this is possible, for example, using local helioseismology (Gizon et al.2010).

Brown et al. (1989) showed that it is convenient to express $\Omega(r, \theta)$ in chosen polynomials in $\cos ^{2} \theta$. Following up on the work of Brown et al. (1989), Ritzwoller and Lavely (1991) and Pijpers (1997) expanded $\Omega(r, \theta)$ in the form

$$
\Omega(r, u)=\sum_{s=0}^{s_{\max }} \Omega_{s}(r) \psi_{2 s}(u),
$$

where $\psi_{2 s}$ is a polynomial in $u$ of degree $2 s$. They showed that this expansion of the rotation rate and using suitable kernels $K_{n l s}$ lead to the following one-to-one relation between $a$-coefficients and $\Omega_{s}$

$$
2 \pi a_{n l, 2 s+1}=\int_{0}^{1} K_{n l s}(r) \Omega_{s}(r) \mathrm{d} r
$$

where $2 s+1$ is equivalent to $j$ in Eq. (1.2). Therefore, we can relate each odd $a$-coefficient to each coefficient of the expansion of the rotation rate independently. Nowadays, up to 18 odd $a$-coefficients are available in observational data which corresponds to the value of $s_{\text {max }}=17$.

The most common way to obtain the rotation rate is to perform a linear inversion of the frequency splitting data. A linear inversion is formulated such that the unknown parameter that we want to infer has a linear relation with the actual data via the inversion coefficients. In the case of a one-dimensional inversion for rotation rate we have

$$
\bar{\Omega}_{s}\left(r_{0}\right)=2 \pi \sum_{n, l} c_{n l s}\left(r_{0}\right) a_{n l, 2 s+1},
$$

where $\bar{\Omega}_{s}$ is the inferred rotation rate at target radius $r_{0}$. Here, $c_{n l s}$ is an inversion coefficient that determines how much each different splitting coefficient contributes to the inferred rotation rate. By substituting Eq. (1.7) into Eq. (1.8) we obtain

$$
\bar{\Omega}_{s}\left(r_{0}\right)=\int_{0}^{1} \mathcal{K}_{s}\left(r_{0} ; r\right) \Omega_{s}(r) \mathrm{d} r
$$


where

$$
\mathcal{K}_{s}\left(r_{0} ; r\right)=\sum_{n, l} c_{n l s}\left(r_{0}\right) K_{n l s}(r)
$$

are the so-called averaging kernels. As in this thesis I use the Multiplicative Optimally Localized Averaging (MOLA) method, I only explain this method and refer the reader to the work by Christensen-Dalsgaard et al. (1990) and Schou et al. (1994) for details about this and other methods for inverting helioseismic data. In the MOLA method, the inversion coefficients are determined such that the averaging kernels are localized at the target radius $r_{0}$ by minimizing

$$
\int_{0}^{1}\left(r-r_{0}\right)^{2} \mathcal{K}_{s}^{2}\left(r_{0} ; r\right) \mathrm{d} r+\mu \sigma^{2}\left[\bar{\Omega}_{s}\left(r_{0}\right)\right]
$$

subject to $\int_{0}^{1} \mathcal{K}_{s}\left(r_{0} ; r\right) \mathrm{d} r=1$. Here, $\mu$ is a trade-off parameter and $\sigma$ is the standard deviation on the inferred rotation rate given by

$$
\sigma^{2}\left[\bar{\Omega}_{s}\left(r_{0}\right)\right]=4 \pi^{2} \sum_{n, l} c_{n l s}^{2}\left(r_{0}\right) \sigma^{2}\left(a_{n l, 2 s+1}\right)
$$

where the errors on $a$-coefficients are assumed to be uncorrelated. The trade-off parameter is a free parameter of this method and it is determined such that it optimizes the localization of the averaging kernels at the expense of magnifying the errors in the inferred rotation rate.

\subsection{Solar differential rotation}

In the last fifty years, helioseismology has shed light on the previously unknown interior rotation profile of the Sun. Before I explain what we have learned about the interior rotation of the Sun, I introduce what we have already known about the surface rotation from a few hundred years observation of the surface of the Sun.

\subsubsection{Surface rotation}

With the invention of telescopes in the 17th century, it became possible to observe the Sun in closer detail. It was discovered that the Sun rotates by observing the sunspots rotating across the solar disk. In 1612, Galileo Galilei reported that it takes 14 days for a spot to traverse the solar disk. In 1630, Christoph Scheiner published the first monograph in solar physics about his observations of sunspots where he estimated the solar rotation period to be about 27 days. He also reported that spots farther from the equator rotate slower than the ones close to the equator.

In the 1850s, Richard Carrington and Gustav Spörer made intensive observations of sunspots. By tracing the spots at different latitudes, they could obtain the first empirical formula for the surface differential rotation (Tassoul and Hadrava 1983). Nowadays, this method of measuring the surface rotation is known as feature tracking, which can use any resolved feature at the surface of the Sun. Beck (2000) compared the rotation rate obtained from different tracers which is shown in Fig. 1.7. I note here that an important assumption for obtaining the surface plasma rotation rate using a tracer motion is that the tracer is co-rotating with the surface plasma. 


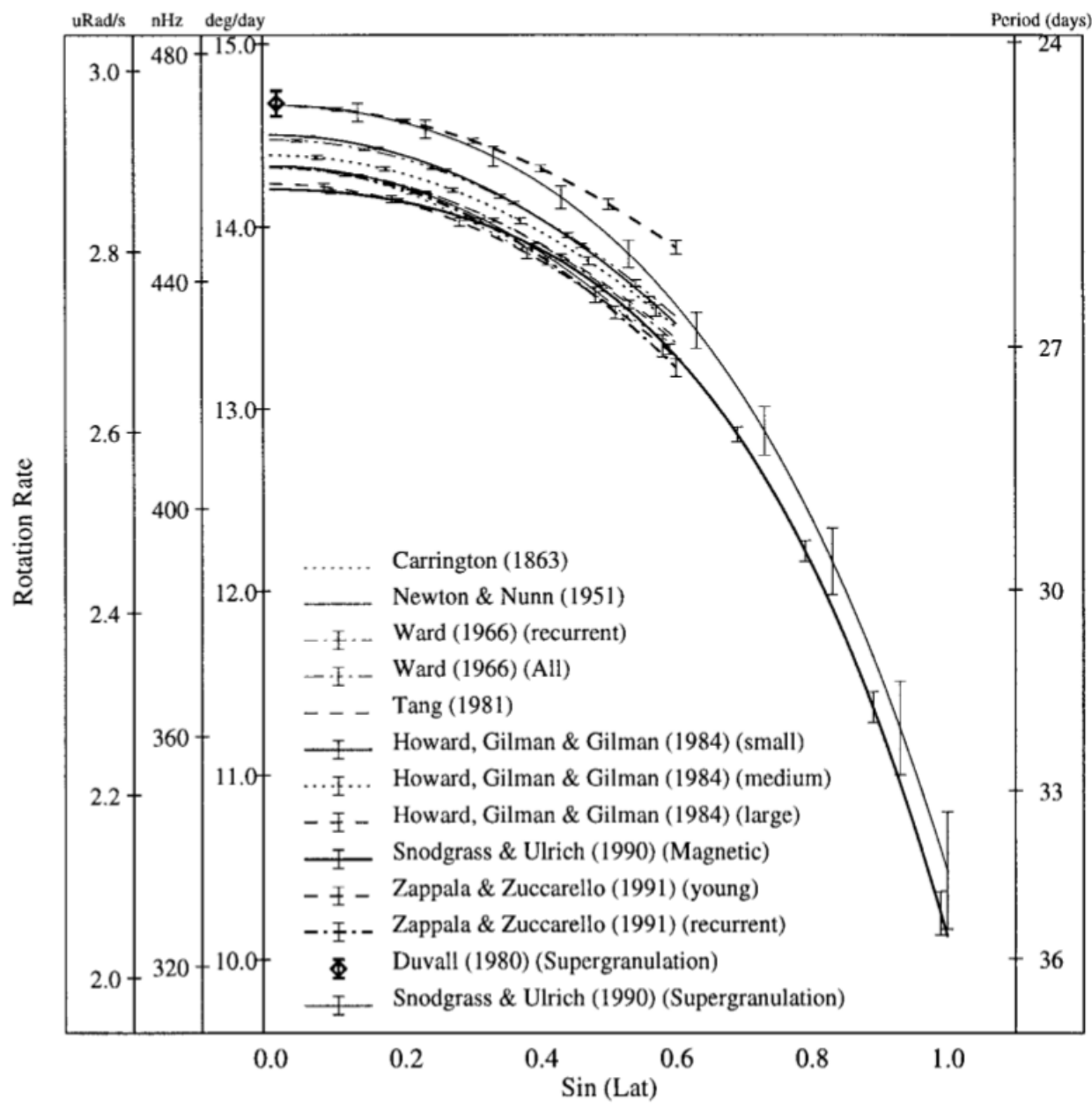

Figure 1.7: Surface rotation rate of the Sun versus sine latitude using different tracers (Beck 2000, reproduced by permission). The references in these two panels are discussed in Beck (2000).

A different way of measuring the solar rotation is to use spectroscopy (Delury 1939. Plaskett 1959, Livingston 1969. Howard and Harvey 1970). This technique is based on measuring the Doppler shift of spectral lines in the solar spectrum. With this method, the rotation rate of the surface plasma can be measured more directly and is often expressed as

$$
\Omega=A+B \cos ^{2}(\theta)+C \cos ^{4}(\theta),
$$

where the parameters $A, B$ and $C$ are obtained by fitting this function to the measured velocities at each latitude. The parameter $A$ represents the equatorial rotation rate and $B$ and $C$ represent the differential rotation. Rotation rates obtained from this method by different authors are also shown in Fig. 1.8. By comparing the rotation rate obtained from surface plasma and tracing sunspots, Howard and Harvey (1970) found that small spots rotate about $2 \%$ faster than the actual mean rotation of the surface plasma. This finding has been confirmed by several other measurements and can clearly be seen in Fig. 1.7.

The faster rotation rate of the magnetic features violates the assumption of co-rotating magnetic features. In the 1970s, the idea of anchoring sunspots in the deeper layers in 


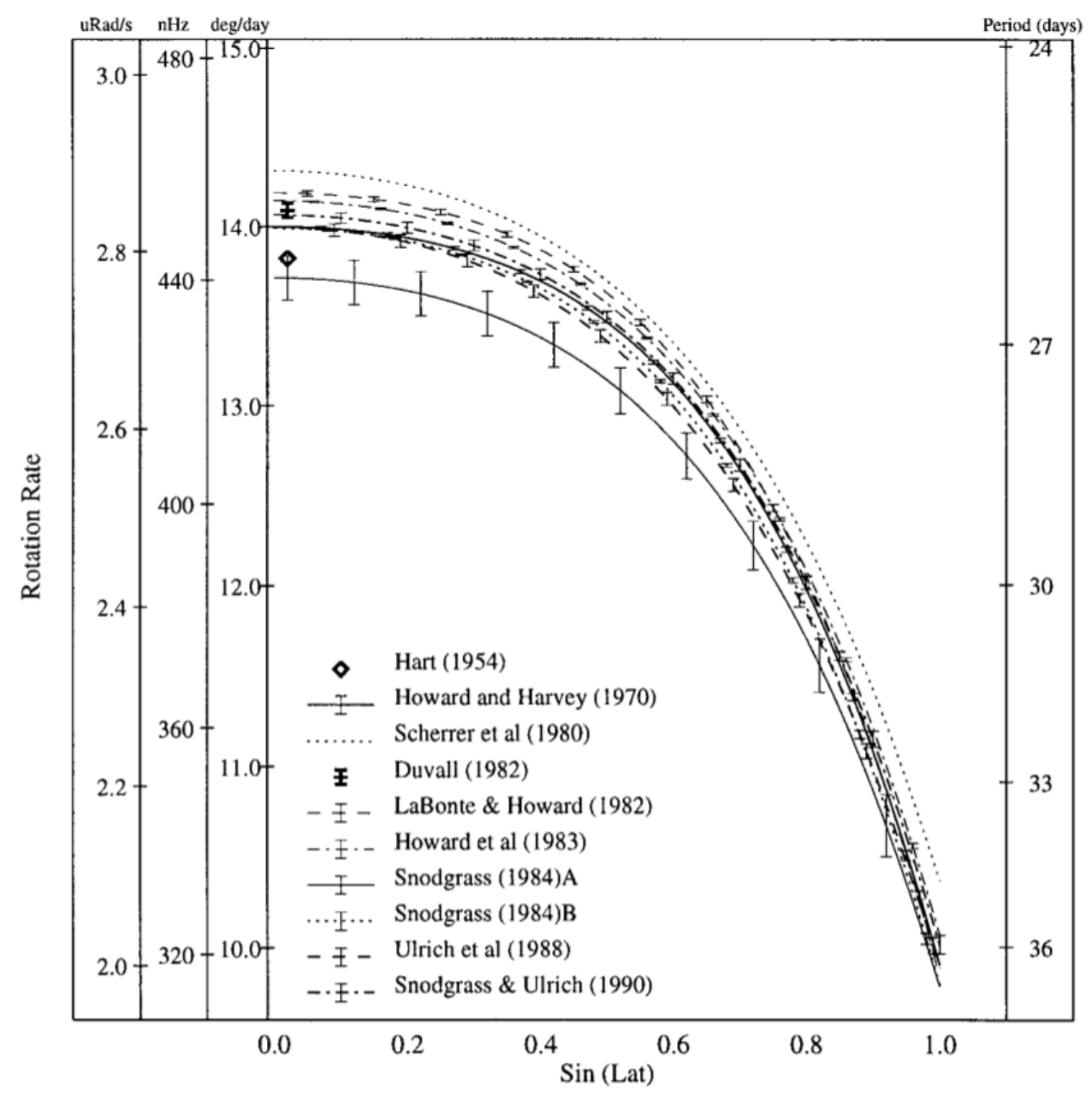

Figure 1.8: Spectroscopic measurements of the surface rotation rate of the Sun versus sine latitude (Beck 2000, reproduced by permission). The references in the figure are discussed in Beck (2000).

which they represent the velocity at those depths was proposed. If that were true, it would mean that there should be layers in the interior that rotate faster than the surface (Foukal 1977).

Theoretical explanations of the observed differential rotation of the Sun have been a great challenge. In particular, it was difficult to explain how the angular momentum is transported from the poles to the equator. This problem was overcome by introducing the non-diffusive part of the Reynolds stress known as the " $\Lambda$-effect" in the angular momentum evolution equation (Rüdiger 1989). According to the Taylor-Proudman theorem, it was believed that the convection zone of the Sun should rotate with contours of constant rotation being on cylinders parallel to the rotation axis of the Sun and that the rotation rate should decrease with depth (see the review by Gilman (1974)). I note here that the proposed idea of anchoring the spots at deeper layers was in opposition to these predictions. 


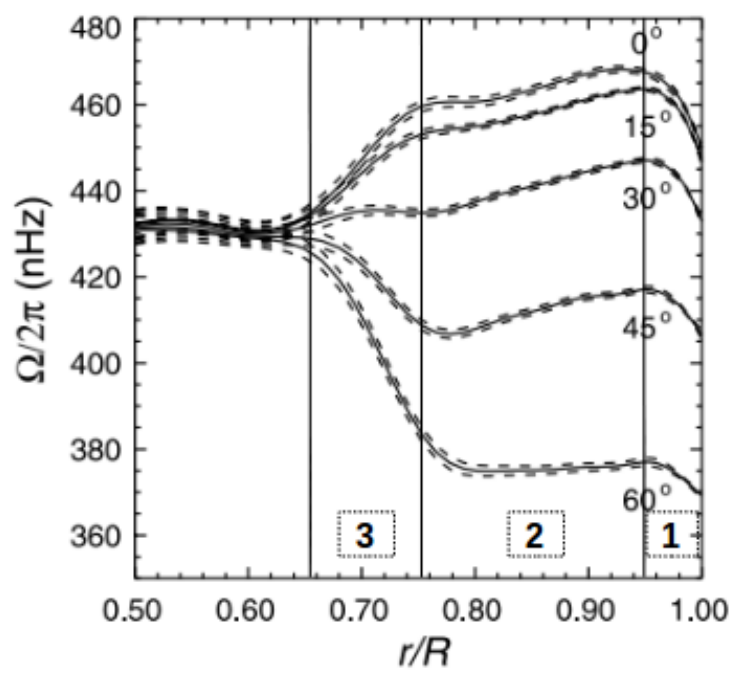

Figure 1.9: Four-year (1995-1999) time average of the rotation rate versus fractional radius at different latitudes obtained from a two dimensional inversion of Global Oscillation Network Group data. The regions 1, 2 and 3 indicate the different layers of distinct radial gradient discussed in the main text (Howe et al. 2000b, reproduced by permission).

\subsubsection{Internal rotation}

The internal rotation profile of the Sun obtained by helioseismology is shown in Fig. 1.9. These measurements show that the radiative zone rotates rigidly with a rotation rate similar to the surface mid-latitude rotation rate. The convection zone rotates differentially in both latitude and radius. In latitude, the rotation rate of the convection zone is similar to the surface rotation in the sense that the rotation rate decreases from the equator towards the poles. In the radial direction, the convection zone can be divided into three regions according to the radial gradient of the rotation rate. These three regions are shown in Fig. 1.9. Region 1 is located close to the surface between approximately $0.95 R_{\odot}$ and the surface and has a strong negative gradient. This layer is known as the near-surface shear layer (NSSL). The value of the gradient is about -400 to $-300 \mathrm{nHz} R_{\odot}^{-1}$ at low latitudes (Antia et al. 2008).

Region 2 is located between approximately $0.75 R_{\odot}$ and $0.95 R_{\odot}$ and has a small positive radial gradient of about 50 to $60 \mathrm{nHz} R_{\odot}^{-1}$. Region 3 is a thin layer located between the convection and radiative zones known as the tachocline (Spiegel and Zahn 1992). This region has a strong positive (negative) gradient at low (high) latitudes. There are several studies investigating the exact location and size of this region. The details of these findings are explained in the book by Hughes et al. (2007).

The theoretical explanation of the differential rotation profile of the Sun became a great challenge as the contours of the constant rotation rate were observed to be spoke-like and not on cylinders parallel to the axis of rotation, as predicted by the differential rotation theory in the 1980's. Kichatinov and Rüdiger (1993) improved the existing model of the differential rotation by taking into account the temperature difference between the equator and the poles. With this theory, they could reproduce several features of the solar 
differential rotation as shown in Kitchatinov and Rüdiger (2005).

\subsection{The near-surface shear layer (NSSL)}

The early analysis of the helioseismic data in the 1970's and 80's provided hints of the existence of the NSSL (Rhodes et al. 1979, 1990, Wilson 1992). The results were not certain because of uncertainties in the observational data. Also, the f modes that can probe shallower layers than $\mathrm{p}$ modes were not observed at that time.

The confident detection of the NSSL using global oscillation modes was reported by Thompson et al. (1996) and Kosovichev et al. (1997) using data from the Global Oscillation Network Group (GONG; Harvey et al. 1996) and MDI, respectively. Schou et al. (1998) compared the rotation profile of the Sun derived from different inversion methods up to $75^{\circ}$ latitude using splittings obtained from 144 days of MDI data. These authors showed that at low latitudes all the inversion methods agreed on the increase of the rotation rate below the surface down to $35 \mathrm{Mm}$, but disagreed at high latitudes. They also showed that at the equator the Sun rotates about $10 \mathrm{nHz}$ faster at $35 \mathrm{Mm}$ depth than at the surface, which corresponds to a negative radial gradient.

This finding was in favor of the possible explanation of the equatorward migration of the activity belt by the Parker-Yushimura sign rule of a propagating dynamo wave (Parker 1955, Yoshimura 1975). The negative radial gradient is required in this model to produce the correct direction of the migration of the activity belt. Moreover, this finding was in agreement with the anchoring of the sunspots in deeper layers that rotate faster than the surface.

Corbard and Thompson (2002) made a detailed analysis to measure the gradient of the NSSL. They used 18 odd $a$-coefficients of $\mathrm{f}$ mode frequency splitting data spanning 1 May 1996 to 4 April 2001. They used angular degrees $117 \leq l \leq 300$, with which they could measure the gradient down to $15 \mathrm{Mm}$ below the surface. They assumed that the rotation rate changes linearly with depth at each latitude. In order to obtain the gradient, they used the relation between the $a$-coefficients and the rotation rate (see Eq. (1.7p) to obtain the average of the rotation rate at each latitude. By performing a linear least square fit of the time average of this rotation rate at each latitude over the central gravity of the radial kernels, they obtained the slope and surface rotation rate. Division of these two values (former by latter) produced the logarithmic radial gradient of the rotation rate. They showed that the logarithmic radial gradient is close to -1 from the equator to $30^{\circ}$ latitude and increases gradually at higher latitudes and changes sign at about $55^{\circ}$ latitude.

At the same time, the $\chi^{2}$ value of their linear fit also increases toward higher latitudes. By further investigation, they speculated that the increase in $\chi^{2}$ at higher latitudes is because of the systematic errors in high degree modes probing the outer $5 \mathrm{Mm}$. This depth corresponds to modes with a degree $l>250$. They repeated their analysis with the modes restricted to $160 \leqslant l \leqslant 250$ and they showed that the gradient stays negative and closer to -0.5 rather than -1 .

Howe et al. (2006) used a local helioseismology technique known as ring diagram analysis (Hill 1988) applied to both GONG and MDI data to measure the radial gradient of the NSSL. Their results were good in agreement with the results obtained by Corbard and Thompson (2002) at low latitudes, but not in good agreement above about $40^{\circ}$. 


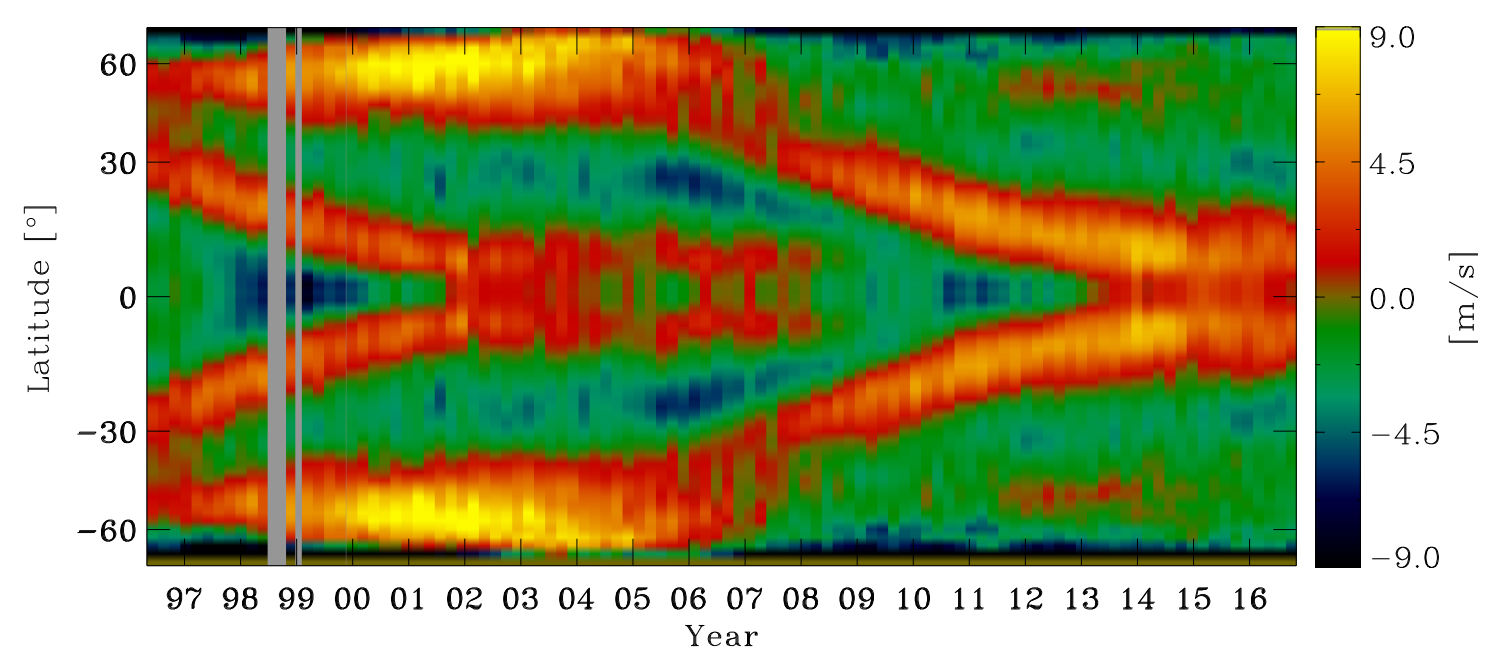

Figure 1.10: Time variation of the zonal flow obtained from $\mathrm{f}$ mode frequency splittings of Medium- $l$ MDI (1996-2010) and HMI (2010-2016) 72-day time series data obtained by subtracting the time averaged value of $a_{1}, a_{3}$ and $a_{5}$ (Courtesy of J. Schou).

\subsection{Time variation of the differential rotation}

Several studies have measured the time variation of the rotation rate at different depths and latitudes (Howard and Labonte 1980, Woodard and Libbrecht 1993, Giles et al. 1998, Schou 1999, Howe et al. 2000c, Toomre et al. 2000, Antia and Basu 2000, Howe et al. 2006, Komm et al.2014). All these studies obtained the variation in the rotation rate over time, either by subtracting the time averaged value of the rotation rate or subtracting a fit of the time averaged rotation using Eq. (1.13) from the actual obtained rotation. I note that the latter approach is roughly equivalent to subtracting the time average of the first three $a$-coefficients from each data set. The variation of residuals over solar cycle is known as the torsional oscillation (Howard and Labonte 1980). An example of measured zonal flow is shown in Fig. 1.10. This figure is an updated version of Fig. 5 of Schou (1999), using data sets of MDI and HMI from 1996 to 2016. Before I describe what we see in this figure, I explain briefly how this figure was obtained as I follow a similar method used in this work for my measurements in the next two chapters.

Schou (1999) used 18 odd $a$-coefficients of f modes obtained from 72-day time series to measure the rotation rate at each latitude around $0.99 R_{\odot}$. He assumed that the rotation rate is independent of radius and expanded it in latitude following Pijpers (1997). He also used the kernels introduced in Pijpers (1997) and performed the MOLA inversion to infer the rotation rate at different latitudes. The resulting averaging kernels of his inversion at different target latitudes $u_{0}$ are shown in Fig. 1.11 . He obtained the zonal flow by subtracting the time averaged value of $a_{1}, a_{3}$ and $a_{5}$ from each data set. In Fig. 1.12, I show the time variation of three averaged $a$-coefficients obtained from 360-day time series of Medium- $l$ MDI (1996-2010) and HMI (2010-2015) data. The coefficient $a_{1}$, which is

\footnotetext{
2 These averaging kernels are identical to the ones used in the analysis in Section 2 and Section 3
} 


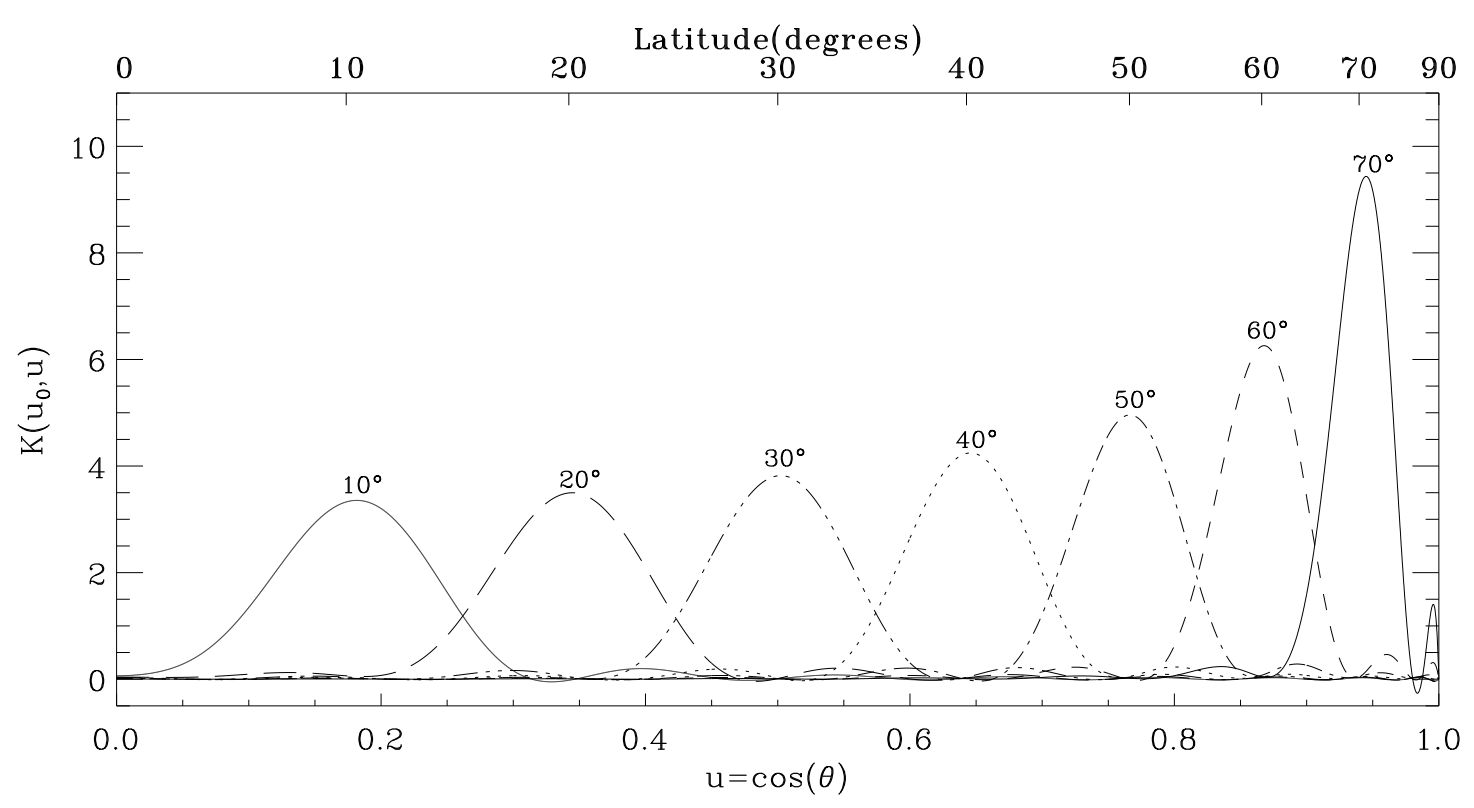

Figure 1.11: Latitudinal averaging kernels versus cosine of colatitude. Different line styles show the averaging kernels at different target $u_{0}$ marked by corresponding latitude.

related to the angular momentum of the Sun, and $a_{5}$ show a periodicity of 11 years, but it seems that $a_{3}$, which corresponds to faster rotation at the equator than the poles, shows a 22-year periodicity. This can be confirmed by having observations of the full cycle 24 .

Now, I come back to the figure of the measured zonal flow. As shown in Fig. 1.10, there are systematic changes in the rotation rate over time and latitude. There are bands of faster and slower than average rotation propagating both toward the equator and toward the poles. As one can see the amplitude of the poleward branch of the faster than the average flow is much weaker in solar cycle 24 than 23 . Moreover, the propagation of the zonal flow toward the equator is similar to the equatorward migration of the activity belt. I note here that the magnitude of the zonal flow varies, depending on what background flow is subtracted.

Howe et al. (2000a) and Antia and Basu (2000) measured the radial extent of this pattern and found out that it penetrates from the surface to $0.9 R_{\odot}$. Howe et al. (2005) measured the zonal flow down to $0.84 R_{\odot}$ and suggested that the torsional oscillation penetrates into the entire convection zone.

\subsection{Thesis motivation}

There are several unsolved problems regarding the subsurface dynamics of the Sun that motivate this study. First, the mechanism generating the NSSL is not well understood and more accurate measurements of the radial gradient of the rotation rate may help constraining theoretical models. For example, the model proposed by Kitchatinov and Rüdiger (2005) predicts no latitudinal dependence of the radial gradient. However, measurements of Corbard and Thompson (2002) showed that there does exist a latitudinal dependence. 

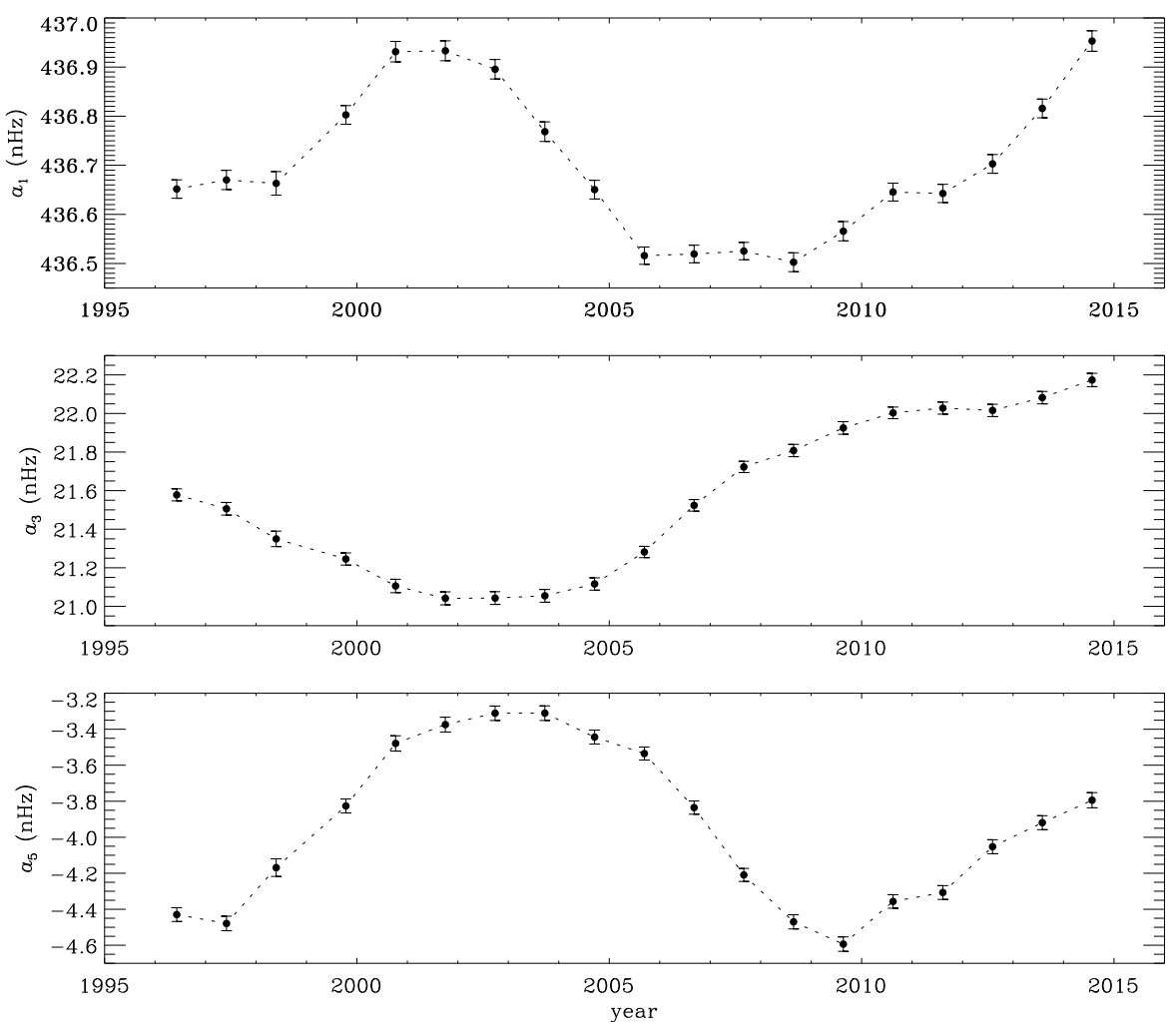

Figure 1.12: The $l$-averaged value of $a_{1}, a_{3}$ and $a_{5}$ as a function of time. The $a$-coefficients were obtained from 360-day of Medium-l MDI (1996-2010) and HMI (2010-2015) time series.

Therefore, it is important to investigate the radial rotation gradient as a function of latitude.

A second motivation for this study is related to the importance of the near-surface shear in dynamo models that try to explain the 11-year cycle of the Sun. Differential rotation is an important ingredient in dynamo theory as it affects the amplification of the poloidal field and its conversion into a toroidal field. This effect, known as the $\Omega$-effect in an $\alpha \Omega$ dynamo model (Brandenburg and Subramanian 2005, Charbonneau 2010) is often believed to be located at the tachocline. However, there are several studies that highlight the role of the NSSL in different dynamo models (Dikpati and Charbonneau 1999, Mason et al.2002, Käpylä et al. 2006, Karak and Cameron 2016). Therefore, a quantitative measure of the values of the radial gradient near the surface can provide constraints in some models on the amount of the toroidal flux that can be produced in the NSSL.

A third motivation is that since the work by Corbard and Thompson (2002) much better quality data became available in two ways. Firstly, several corrections have been applied to the analysis of the data that lead to reduced systematic errors in the measurements of $\mathrm{f}$ mode frequencies and consequently in $a$-coefficients (Larson and Schou 2015). For example, a yearly periodicity of the $\mathrm{f}$ mode frequencies of high degree modes was removed successfully. Secondly, the improved pipeline for obtaining $a$-coefficients was applied to 360-day time series in addition to 72-day ones which became available in 2015. The major difference between these data sets and the ones obtained from 72-day time series is that more lower degree modes have been analyzed successfully as these data have better 
frequency resolution. Therefore, it is important to measure the gradient using the better quality data as they might have an impact on the change of the gradient at $55^{\circ}$ latitude reported by Corbard and Thompson (2002).

Lastly and most importantly, this work provides a uniform analysis of rotational splittings from almost 20 years (1996-2015) of observations, thus, giving an opportunity to investigate the effect of the solar cycle variation on the radial gradient close to the surface of the Sun. Such an attempt has already been made by Antia et al. (2008) who measured the rotation rate in the entire convection zone and its temporal variation in solar cycle 23 including the NSSL. They used 12 years (1995-2007) of GONG and 11 years (1996-2007) of MDI data. However, they only performed their analysis up to $0.98 R_{\odot}$. They excluded shallower layers as there were discrepancies in the results obtained from GONG and MDI data. Measurements of the change of the differential rotation with activity cycle of the Sun can be important for theories of the differential rotation. It is believed that the differential rotation is the outcome of the interplay between the rotation and convection. However, the effect of the magnetic field has been ignored so far, but it may play an important role. This study may provide useful information about the role of the magnetic field and its effect on the rotation rate close to the surface of the Sun. 



\title{
2 Paper I: The radial gradient of the near-surface shear layer of the Sun
}

\begin{abstract}
Helioseismology has provided unprecedented information about the internal rotation of the Sun. One of the important achievements was the discovery of two radial shear layers: one near the bottom of the convection zone (the tachocline) and one near the surface. These shear layers may be important ingredients for explaining the magnetic cycle of the Sun. We measure the logarithmic radial gradient of the rotation rate $(\mathrm{d} \ln \Omega / \mathrm{d} \ln r)$ near the surface of the Sun using 15 years of $\mathrm{f}$ mode rotational frequency splittings from the Michelson Doppler Imager (MDI) and four years of data from the Helioseismic and Magnetic Imager (HMI). We model the angular velocity of the Sun in the upper $\sim 10 \mathrm{Mm}$ as changing linearly with depth and use a multiplicative optimally localized averaging inversion to infer the gradient of the rotation rate as a function of latitude. Both the MDI and HMI data show that $\mathrm{d} \ln \Omega / \mathrm{d} \ln r$ is close to -1 from the equator to $60^{\circ}$ latitude and stays negative up to $75^{\circ}$ latitude. However, the value of the gradient is different for MDI and HMI for latitudes above $60^{\circ}$. Additionally, there is a significant difference between the value of $\mathrm{d} \ln \Omega / \mathrm{d} \ln r$ using an older and recently reprocessed MDI data for latitudes above $30^{\circ}$. We could reliably infer the value of $\mathrm{d} \ln \Omega / \mathrm{d} \ln r$ up to $60^{\circ}$, but not above this latitude, which will hopefully constrain theories of the near-surface shear layer and dynamo. Furthermore, the recently reprocessed MDI splitting data are more reliable than the older versions which contained clear systematic errors in the high degree $\mathrm{f}$ modes ${ }^{1}$.
\end{abstract}

\subsection{Introduction}

Helioseismology has had a significant impact on our understanding of the internal structure and dynamics of the Sun. One of the most important results has been the inference of the rotation profile (Schou et al. 1998). Two shear layers have been identified, one located near the base of the convection zone (Christensen-Dalsgaard and Schou 1988, Brown et al. 1989), known as the tachocline (Spiegel and Zahn 1992), and one in the upper $35 \mathrm{Mm}$, the near-surface shear layer (NSSL, Thompson et al. 1996). Explaining the current picture

\footnotetext{
1 This section is identical to the published article: "The radial gradient of the near-surface shear layer of the Sun", A. Barekat, J. Schou, L. Gizon, Astronomy \& Astrophysics,570, L12, 2014, reproduced with permission of Astronomy \& Astrophysics journal (c) ESO. A. Barekat contributed to performing the research, analyzing the data and writing the article.
} 
of the internal rotation profile in theoretical terms is a major challenge. (Kichatinov and Rüdiger 1993, Kitchatinov and Rüdiger 2005).

The rotation profile in general and shear layers in particular may play a crucial role for the solar dynamo (e.g., Brandenburg and Subramanian 2005, Charbonneau 2010). This led to further investigation of the NSSL using helioseismic measurements (Basu et al. 1999; Corbard and Thompson 2002, hereafter CT; Howe et al. 2006; Zaatri and Corbard 2009) and its role in dynamo theory (Dikpati et al.2002, Mason et al. 2002, Brandenburg 2005, Käpylä et al.2006). The logarithmic radial gradient of the rotation rate $(\mathrm{d} \ln \Omega / \mathrm{d} \ln r)$ evaluated at the surface was measured by CT using f modes. They used 23 data sets (each from 72-day time series) of 18 odd $a$-coefficients from the Medium- $l$ program (Scherrer et al. 1995) of the Michelson Doppler Imager (MDI) onboard the Solar and Heliospheric Observatory (SOHO) covering the years 1996 through 2001. Their main result was that $\mathrm{d} \ln \Omega / \mathrm{d} \ln r \sim-1$ up to $30^{\circ}$ latitude, reverses sign around $55^{\circ}$ latitude and stays positive at higher latitudes. However, they also noted that there are indications of systematic errors mostly affecting high latitudes. We address this issue by analyzing splittings from MDI and the Helioseismic and Magnetic Imager (HMI; Schou et al.2012) onboard the Solar Dynamics Observatory (SDO).

\subsection{Observations}

Thousands of oscillation mode frequencies $v_{n l m}$ can be measured on the Sun, where $n, l$, and $m$ are the radial order, the spherical harmonic degree, and the azimuthal order, respectively. The mode frequencies $v_{n l m}$ are expanded using so-called $a$-coefficients (Schou et al. 1994)

$$
v_{n l m}=v_{n l}+\sum_{j=1}^{j=36} a_{n l, j} \mathcal{P}_{j}^{(l)}(m),
$$

where $v_{n l}$ is the mean multiplet frequency and $\mathcal{P}_{j}^{(l)}$ are orthogonal polynomials of degree $j$. This work considers only f modes, for which $n=0$, and so we suppress $n$ in the following. We use two sets of $a$-coefficients. The first is from the MDI Medium- $l$ program and contains 74 sets of splittings from independent 72-day time series (Larson \& Schou in prep.). These data cover about 15 years from 1996 May 1 to 2011 April 24, except for 1998 from July 2 to October 17 and 1998 December 23 to 1999 February 2 due to technical problems with SOHO. The second set is from HMI and contains 20 sets of splittings from consecutive 72-day time series (Larson \& Schou in prep.), covering four years of observation from 2010 April 30 to 2014 April 8. Additionally, in order to compare our results with the results obtained by CT, we also use an older version of the MDI data. The differences between these versions come from various improvements to the analysis, as described in Larson and Schou (2009) and (Larson \& Schou in prep.). We refer to the older version as "old MDI" and to the latest "new MDI". The f modes we use cover the range $117 \leq l \leq 300$ for MDI and $123 \leq l \leq 300$ for HMI. We note that the number of available modes changes with time because of noise. 

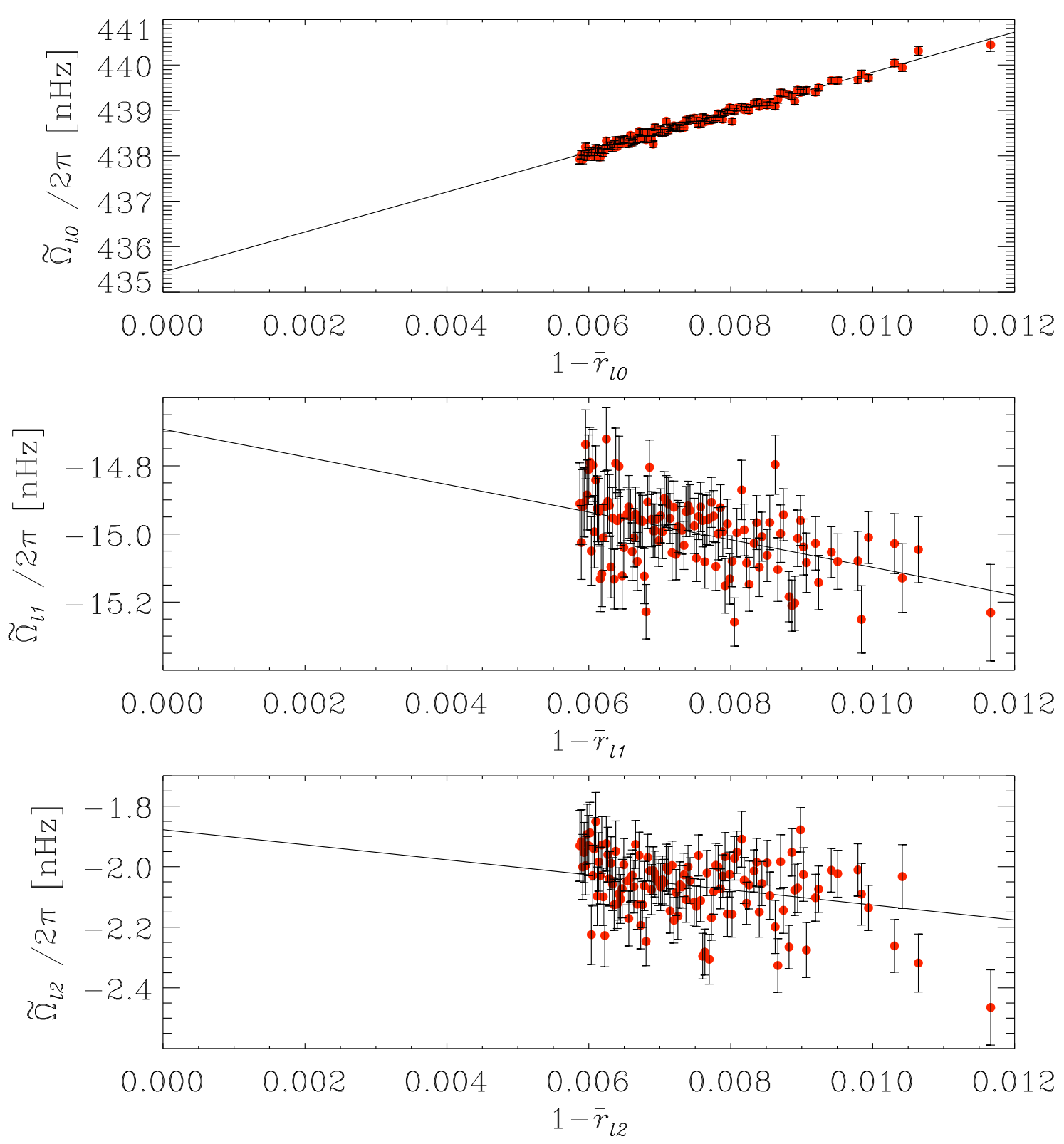

Figure 2.1: $\widetilde{\Omega}_{l s} / 2 \pi$ versus $\left(1-\bar{r}_{l s}\right)$ for $s=0,1$, and 2 from top to bottom for the HMI data set starting on 2014 January 27 . The error bars are $1 \sigma$.

\subsection{Analysis of $f$ mode data}

The odd $a$-coefficients are related to the angular velocity $\Omega$ by

$$
2 \pi a_{l, 2 s+1}=\int_{0}^{1} \int_{-1}^{1} K_{l s}(r, u) \Omega(r, u) \mathrm{d} u \mathrm{~d} r
$$

where the kernels $K_{l s}$ are known functions, $u=\cos \theta, \theta$ is the co-latitude, and $r$ is the distance to the center of the Sun divided by the photospheric radius. Using the results of 
Pijpers (1997), one can show that the kernels can be separated in the variables $r$ and $u$,

$$
K_{l s}(r, u)=F_{l s}(r) G_{s}(u)
$$

where the functions $F_{l s}$ and $G_{s}$ are the radial and latitudinal parts of the kernels. The function $F_{l s}$ is

$$
F_{l s}(r)=\left[F_{l, 1}(r)-F_{l, 2}(r)(2 s+2)(2 s+1) / 2\right] v_{l, 2 s+1},
$$

where $F_{l, 1}, F_{l, 2}$ and $v_{l, 2 s+1}$ are given by

$$
\begin{aligned}
F_{l, 1}(r) & =\rho(r) r^{2}\left[\xi_{l}^{2}(r)-2 \xi_{l}(r) \eta_{l}(r) / L+\eta_{l}^{2}(r)\right] / I_{l}, \\
F_{l, 2}(r) & =\rho(r) r^{2} \eta_{l}^{2}(r) /\left(L^{2} I_{l}\right), \\
v_{l, 2 s+1} & =\frac{(-1)^{s}}{l} \frac{(2 l+1) !(2 s+2) !(l+s+1) !}{s !(s+1) !(l-s-1) !(2 l+2 s+2) !} .
\end{aligned}
$$

In the above equations $\rho$ is the density, $L=\sqrt{l(l+1)}, \xi$ and $\eta$ are the radial and horizontal displacement eigenfunctions as defined by Pijpers (1997), and $I_{l}=\int_{0}^{1} \rho(r) r^{2}\left[\xi_{l}^{2}(r)+\eta_{l}^{2}(r)\right] \mathrm{d} r$. The latitudinal part of the kernels is given by

$$
G_{s}(u)=-\frac{(4 s+3)}{2(2 s+2)(2 s+1)}(1-u)^{1 / 2} P_{2 s+1}^{1}(u),
$$

where $P_{2 s+1}^{1}$ are associated Legendre polynomials of degree $2 s+1$ and order one. As seen later, the form of Eq. 2.3 is useful in that the latitudinal part of the kernels is independent of $l$.

We use f modes to calculate $\mathrm{d} \ln \Omega / \mathrm{d} \ln r$ close to the surface of the Sun in several steps. In the first step, we assume that the rotation rate changes linearly with depth at each latitude

$$
\Omega(r, u)=\Omega_{0}(u)+(1-r) \Omega_{1}(u),
$$

where $\Omega_{1}$ is the slope and $\Omega_{0}$ is the value of the rotation rate at the surface. Combining Eq. (2.9) with Eqs. (3.3) and 2.3) we obtain

$$
\widetilde{\Omega}_{l s} \equiv \frac{2 \pi a_{l, 2 s+1}}{\beta_{l s}}=\left\langle\Omega_{0}\right\rangle_{s}+\left(1-\bar{r}_{l s}\right)\left\langle\Omega_{1}\right\rangle_{s},
$$

where $\beta_{l s}=\int_{0}^{1} F_{l s}(r) \mathrm{d} r$ and $\bar{r}_{l s}=\beta_{l s}^{-1} \int_{0}^{1} F_{l s}(r) r \mathrm{~d} r$ is the center of gravity of $F_{l s}$. The quantities $\left\langle\Omega_{0}\right\rangle_{s}$ and $\left\langle\Omega_{1}\right\rangle_{s}$ are the latitudinal averages

$$
\begin{aligned}
\left\langle\Omega_{0}\right\rangle_{s} & =\int_{-1}^{1} G_{s}(u) \Omega_{0}(u) \mathrm{d} u \\
\left\langle\Omega_{1}\right\rangle_{s} & =\int_{-1}^{1} G_{s}(u) \Omega_{1}(u) \mathrm{d} u .
\end{aligned}
$$

By performing an error weighted linear least squares fit of $\widetilde{\Omega}_{l s}$ versus $\left(1-\bar{r}_{l s}\right)$ we can estimate $\left\langle\Omega_{0}\right\rangle_{s}$ and $\left\langle\Omega_{1}\right\rangle_{s}$. This procedure is applied for all $s$ with $0 \leq s \leq 17$ for each individual 72 -day data set. To illustrate this, Fig. 2.1 shows $\widetilde{\Omega}_{l s} / 2 \pi$ as a function of $\left(1-\bar{r}_{l s}\right)$ for $s=0,1$, and 2 for one time period. We note that the values of $\left(1-\bar{r}_{l s}\right)$ correspond to a 


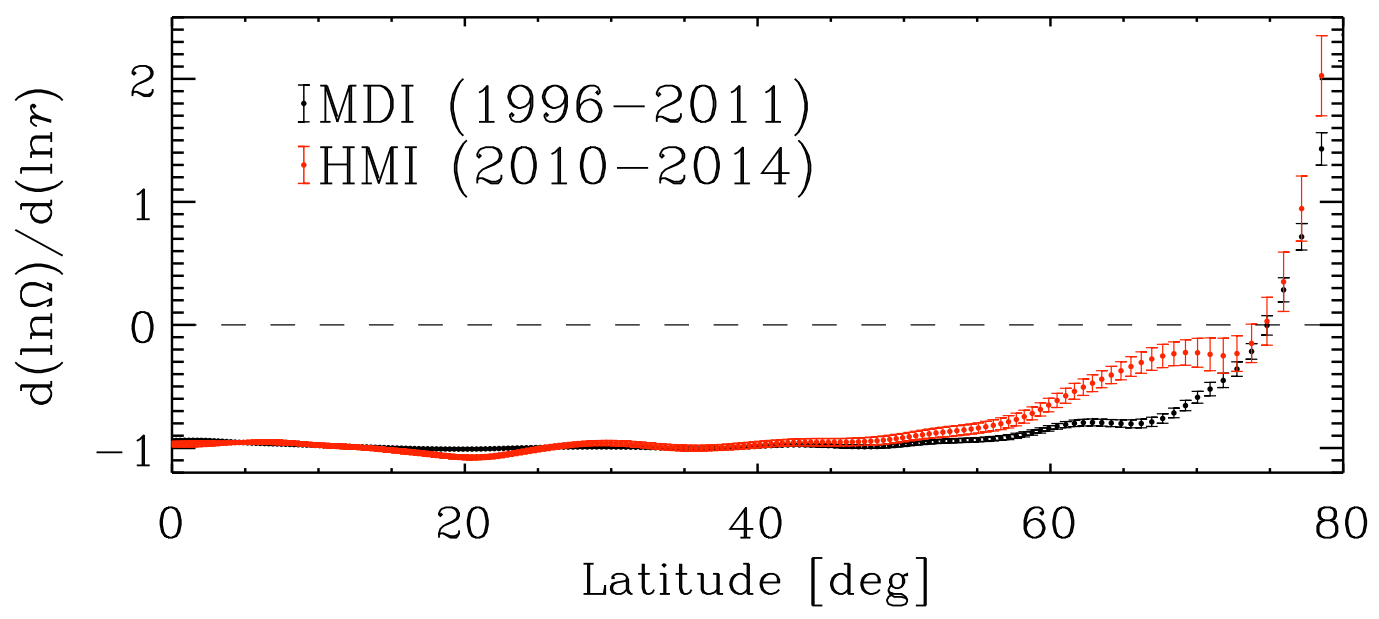

Figure 2.2: Time average of $\mathrm{d} \ln \Omega / \mathrm{d} \ln r$ versus target latitude, obtained from 15 years (1996-2011) of MDI data (black dots) and 4 years (2010-2014) of HMI data (red dots). The error bars are $1 \sigma$.

depth range of about 4.5 - 8.4 Mm, and that the kernels have a significant extent in depth. Our estimates of $\Omega_{0}$ at the surface are thus in effect extrapolations and the values of $\Omega_{1}$ are averages, both estimated from roughly the outer $10 \mathrm{Mm}$.

Next we invert $\left\langle\Omega_{0}\right\rangle_{s}$ and $\left\langle\Omega_{1}\right\rangle_{s}$ to obtain estimates $\bar{\Omega}_{0}\left(u_{0}\right)$ and $\bar{\Omega}_{1}\left(u_{0}\right)$ of $\Omega_{0}\left(u_{0}\right)$ and $\Omega_{1}\left(u_{0}\right)$, where $u_{0}$ is the target point for the inversion. Following $\operatorname{Schou}(1999)$, we use a multiplicative optimally localized averaging inversion method with a trade-off parameter $\mu=0$. This implies that the averaging kernels for $\left\langle\Omega_{0}\right\rangle_{s}$ and $\left\langle\Omega_{1}\right\rangle_{s}$ are the same as those shown in Figure 4 of Schou (1999).

Finally, we obtain an estimate of the surface value of the logarithmic radial gradient of the angular velocity at each $u_{0}$ as

$$
\left(\frac{\mathrm{d} \ln \Omega}{\mathrm{d} \ln r}\right)\left(r=1, u=u_{0}\right) \approx-\frac{\bar{\Omega}_{1}\left(u_{0}\right)}{\bar{\Omega}_{0}\left(u_{0}\right)} .
$$

\subsection{Results}

In Fig. 2.2 we plot the estimates of $d \ln \Omega / d \ln r$ as a function of target latitude ( $\arcsin u_{0}$ ) averaged over time for 15 years of new MDI data and 4 years of HMI data; in Table 2.1 we give the results. The results are similar and very close to -1 from the equator to $\sim 60^{\circ}$ latitude, while above $60^{\circ}$ they diverge. The differences at high latitudes could be due to either systematic errors or a solar cycle effect (the data sets cover different parts of the solar cycle). To investigate this discrepancy, Fig. 2.3 shows the results of applying our method to the HMI and MDI data sets from the five common 72-day periods between 2010 April 30 and 2011 April 24 . The results are consistent up to $\sim 60^{\circ}$ within $2-\sigma$, but show significant inconsistencies at higher latitudes. An analysis using only the common modes and the average errors does not significantly reduce this high latitude discrepancy. This indicates that there are systematic errors in at least one of the data sets, as opposed to only differences in the mode coverage or error estimates. The source of the systematic 
Table 2.1: Selected values of $\mathrm{d} \ln \Omega / \mathrm{d} \ln r$ from Fig. 2.2.

\begin{tabular}{ccc}
\hline \hline Latitude [deg] & MDI & HMI \\
\hline 0 & $-0.939 \pm 0.009$ & $-0.97 \pm 0.02$ \\
10 & $-0.981 \pm 0.007$ & $-0.98 \pm 0.02$ \\
20 & $-1.009 \pm 0.007$ & $-1.08 \pm 0.02$ \\
30 & $-0.992 \pm 0.009$ & $-0.96 \pm 0.02$ \\
40 & $-0.986 \pm 0.011$ & $-0.97 \pm 0.03$ \\
50 & $-0.974 \pm 0.014$ & $-0.92 \pm 0.03$ \\
60 & $-0.841 \pm 0.022$ & $-0.65 \pm 0.05$ \\
70 & $-0.588 \pm 0.048$ & $-0.23 \pm 0.12$ \\
\hline
\end{tabular}

errors is unknown, but could be related to inaccurate estimates of the optical distortion of the instruments or similar geometric errors (Larson \& Schou in prep.). Another possible source is the different duty cycles. For example, the last three data sets for MDI had duty cycles of $88 \%, 73 \%$, and $81 \%$, while the corresponding HMI duty cycles were $97 \%, 99 \%$, and $96 \%$. In either case we conclude that the results above $\sim 60^{\circ}$ should be treated with caution.

The results presented here are significantly different from those obtained by CT. They found that $\mathrm{d} \ln \Omega / \mathrm{d} \ln r$ is close to -1 from the equator to $30^{\circ}$ latitude, while our result shows this up to $60^{\circ}$ latitude. They also found that their results changed significantly if they restricted the degree range. To investigate the origin of these differences we examine the effects of each of the differences between their data and analysis and ours.

First, we compare the results of applying our method and theirs to the 23 time periods they used (covering the period 1996 May 1 to 2001 April 4). Corbard and Thompson (2002) first made an error weighted time average of an older version of the MDI data and then applied their Eq. (9). If we repeat this procedure on the same data sets we obtain results visually identical to theirs. The difference between the data sets used by CT and old MDI is that a few modes were accidentally removed from the older set. We then changed the processing order to first apply their Eq. (9) to old MDI and then make an unweighted time average. As shown in Fig. 2.4, this results in minor differences at high latitude and an analysis applying each change separately shows that only the change from weighted fits to unweighted fits leads to a noticable difference.

We then restricted the old MDI mode set to $160 \leqslant l \leqslant 250$. As shown in Fig. 2.4, this results in large changes above $\sim 50^{\circ}$, in agreement with what $\mathrm{CT}$ found. This indicates that the linear model of the rotation rate (as given by Eq. (2.9)) is incorrect or that there are systematic errors.

Finally, we apply our method to the old MDI and new MDI data sets. As can be seen in Fig. 2.4 we see a significant difference above $30^{\circ}$ latitude. The result using the new MDI data does not show any change of the sign up to $\sim 55^{\circ}$ latitude and is $\sim-1$ up to $60^{\circ}$ latitude. The results using the new MDI data sets also show good agreement between the results of the complete and restricted mode sets up to almost $70^{\circ}$ latitude, indicating that the model of linear change of the angular velocity with depth represents those data better than the old MDI data. 

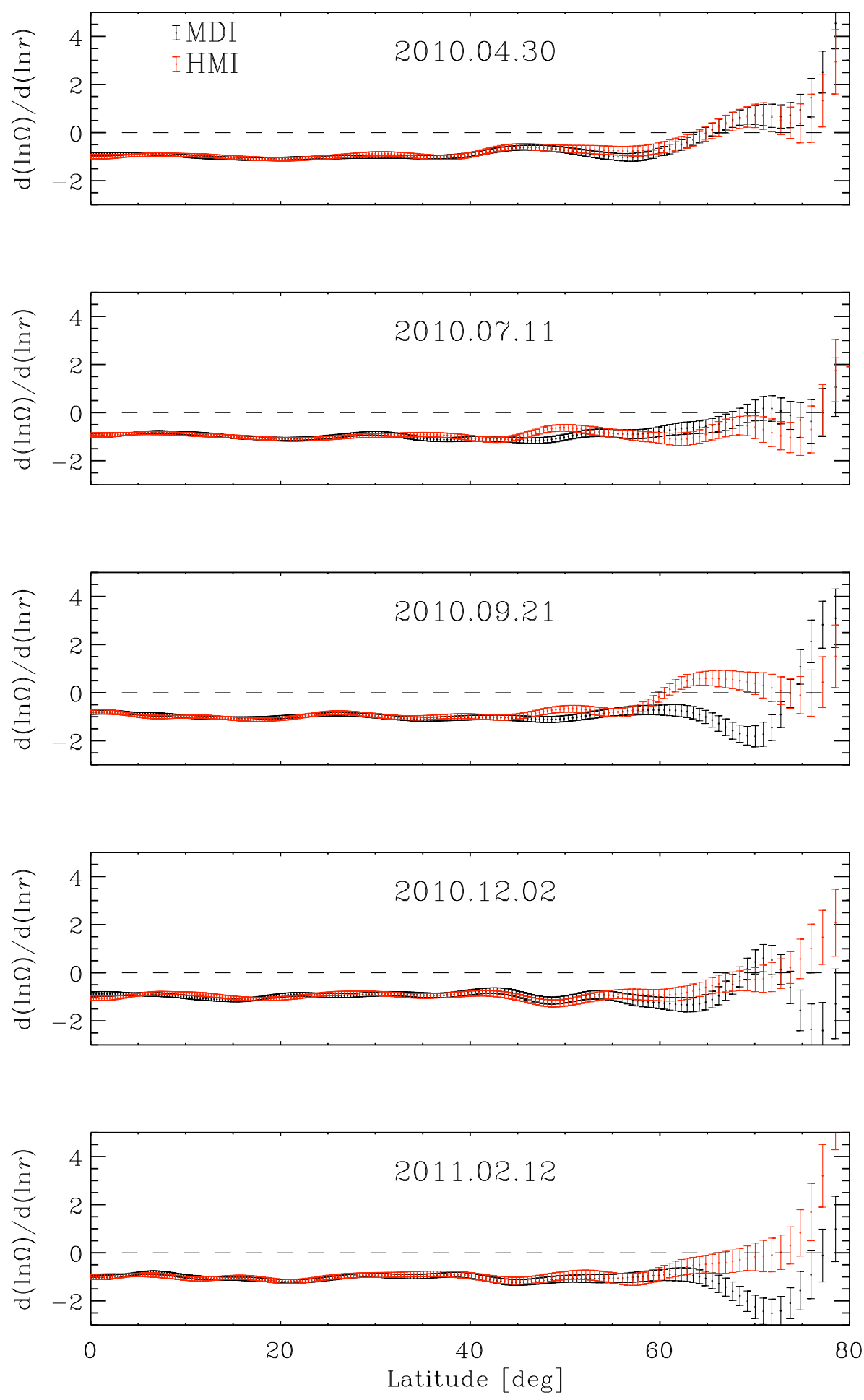

Figure 2.3: Comparison of $\mathrm{d} \ln \Omega / \mathrm{d} \ln r$ versus target latitude for MDI (black dots) and HMI (red dots) from the five common 72-day time series (indicated by the nominal beginning dates). Error bars are 1- $\sigma$. 


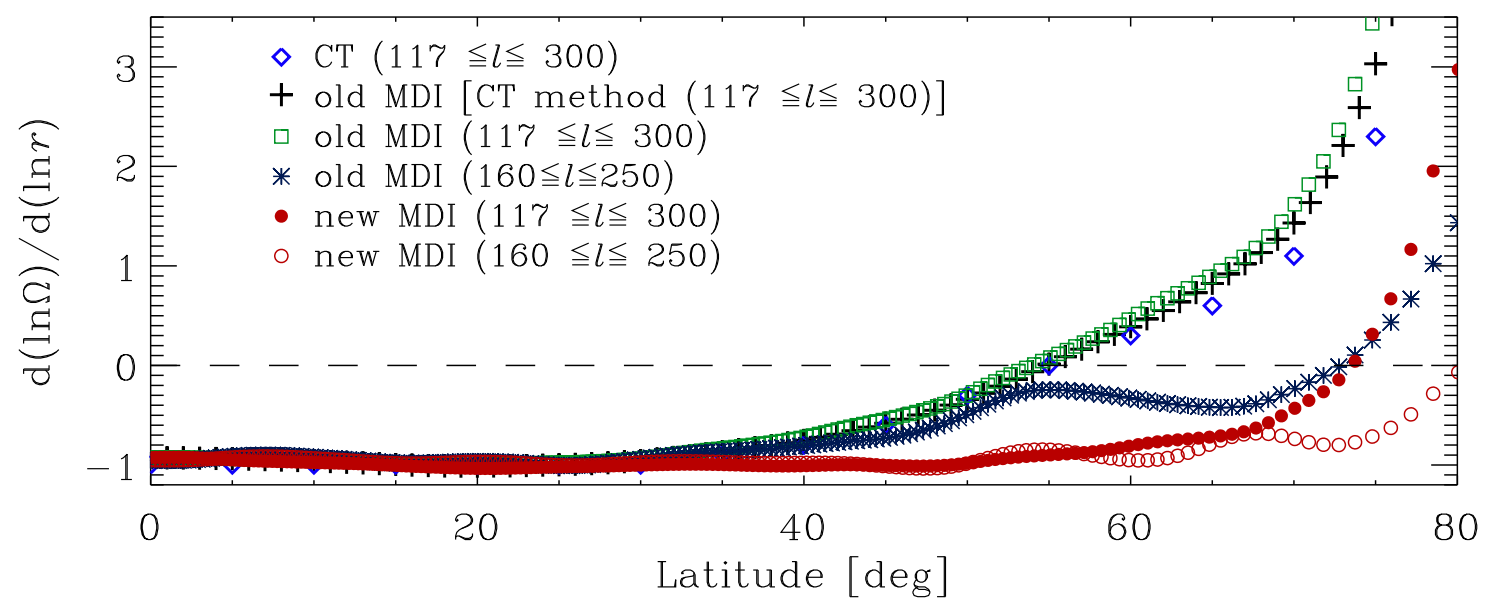

Figure 2.4: Estimates of $d \ln \Omega / d \ln r$ versus target latitude obtained from 23 MDI data sets using various methods. Blue diamonds show the values measured from Fig. 4 of CT, while black pluses show the results of changing the data sets and averaging, as described in the text. Green squares and dark blue stars show the results of our analysis of the old MDI data for the full and restricted modes, respectively. Filled and open red circles show the corresponding results for the new MDI data.

As almost all the differences between the results obtained by $\mathrm{CT}$ and ours come from the differences between old and new MDI, we compare the $a$-coefficients directly. As an example, Fig. 2.5 shows $a_{3}$ for the modes with $150 \leqslant l \leqslant 300$ for all 74 periods. The main differences between new and old MDI appear for $l>270$. In the new MDI data most of the missing modes (shown in black) in the old MDI data are recovered and the yearly oscillatory pattern disappears. These differences clearly show that the old MDI data have significant systematic errors in the high degree $f$ modes. We also note that the new values of $a_{3}$ are shifted toward higher values.

\subsection{Conclusion}

We analyze 15 years (1996-2011) of reprocessed MDI data and 4 years (2010-2014) of HMI data to infer the logarithmic radial gradient of the angular velocity of the Sun in the upper $\sim 10 \mathrm{Mm}$. By using data from two instruments and applying a different method than $\mathrm{CT}$ did, we confirm their value of $\mathrm{d} \ln \Omega / \mathrm{d} \ln r \sim-1$ at low latitudes $\left(<30^{\circ}\right)$; unlike CT, we show that $\mathrm{d} \ln \Omega / \mathrm{d} \ln r$ stays nearly constant and close to -1 up to $60^{\circ}$ latitude. With further analysis we conclude that the inconsistency between their results and ours for latitudes above $30^{\circ}$ is due to systematic errors in the old MDI data. This implies that work done using old MDI data should be revisited. By comparing the results obtained from new MDI and HMI data, we also conclude that at least one of the data sets is likely still suffering from some systematic errors which leads to the discrepancy above $60^{\circ}$ latitude.

The measured value $\mathrm{d} \ln \Omega / \mathrm{d} \ln r \sim-1$ is inconsistent with the standard picture of angular momentum conservation where $\mathrm{d} \ln \Omega / \mathrm{d} \ln r$ is -2 (Foukal 1977, Gilman and Foukal 1979). More recently, hydrodynamical mean-field simulations of a larger part of 

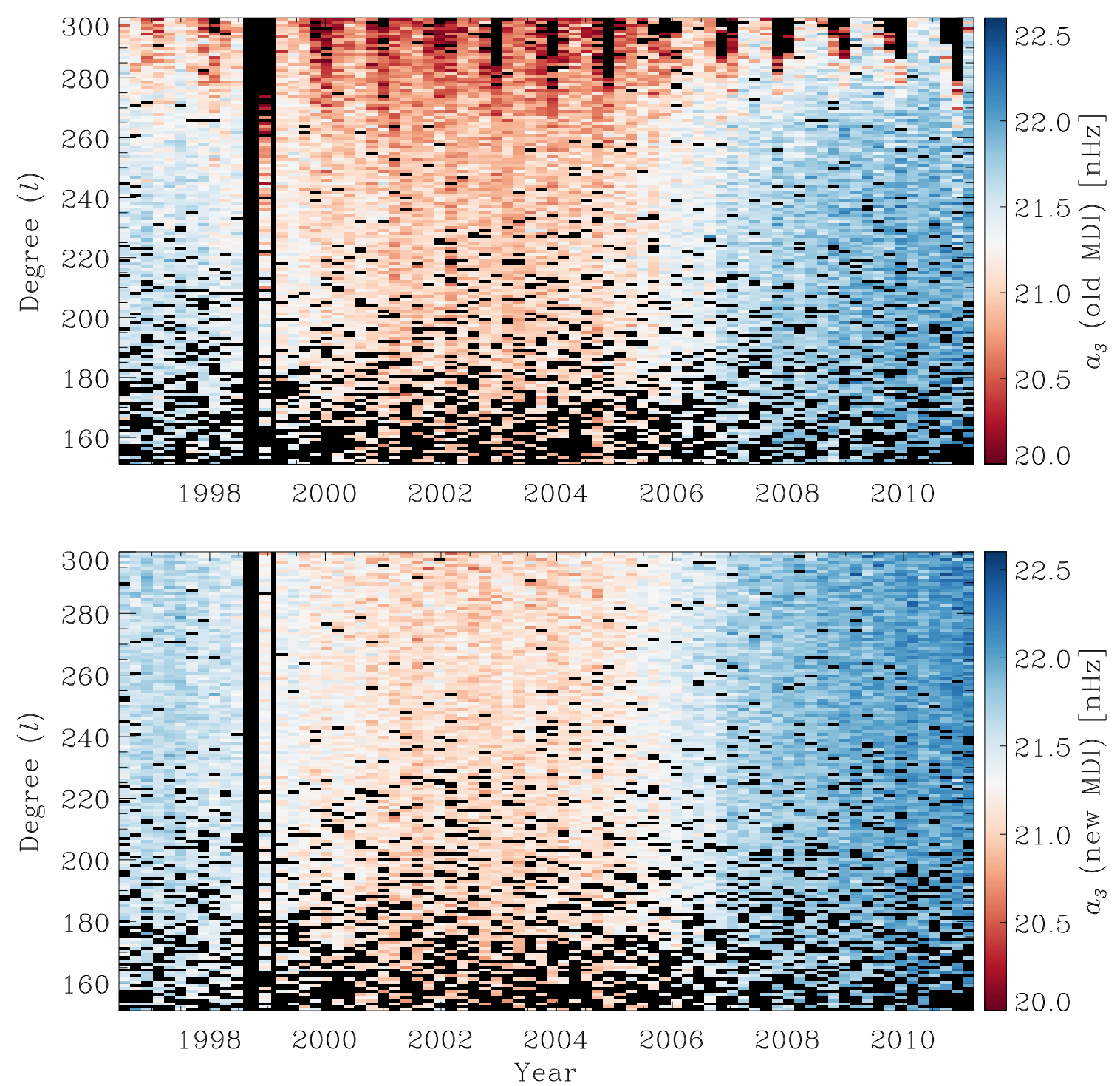

Figure 2.5: $a_{3}$ for old MDI (upper panel) and new MDI (lower panel) for $150 \leqslant l \leqslant 300$ over time. Black shows missing modes. For clarity a few old MDI values below $20 \mathrm{nHz}$ were set to $20 \mathrm{nHz}$.

the convection zone by Kitchatinov and Rüdiger (2005) show a NSSL with a negative radial gradient of the angular velocity from the equator to $80^{\circ}$ latitude. Their theory (Kichatinov and Rüdiger 1993, Kitchatinov and Rüdiger 1999, Kitchatinov 2013) states that the formation of the NSSL is due to the balance of the $\Lambda$-effect (Rüdiger 1989) and the eddy viscosity. However, producing a NSSL with the correct radial gradient remains a challenge for direct numerical simulations of the Sun (e.g., Warnecke et al.|2013, Guerrero et al.2013) and we still do not understand why the value of $d \ln \Omega / d \ln r$ at the surface is nearly constant and so close to -1 .

We note here that we measure $\mathrm{d} \ln \Omega / \mathrm{d} \ln r$ only in the upper $\sim 10 \mathrm{Mm}$ which is only about one third of the NSSL. To extend this range one would need to use $\mathrm{p}$ modes, which 
unfortunately have much more noise. A preliminary analysis shows that $\mathrm{d} \ln \Omega / \mathrm{d} \ln r$ shows little solar cycle variation, though there are weak hints of a torsional oscillation-like signal. However, this requires further analysis.

\section{Acknowledgements}

We thank T. P. Larson for discussions regarding details of the MDI and HMI peakbagging, T. Corbard and M. J. Thompson for clarifying details of their analysis, and A. Birch for various discussions. SOHO is a project of international cooperation between ESA and NASA. The HMI data are courtesy of NASA/SDO and the HMI science team. 


\title{
3 Paper II: Solar-cycle variation of the rotational shear near the solar surface
}

\begin{abstract}
Helioseismology has revealed that the angular velocity of the Sun increases with depth in the outermost $35 \mathrm{Mm}$ of the Sun. Recently, we have shown that the logarithmic radial gradient $\left(\mathrm{d} \ln \Omega / \mathrm{d} \ln r\right.$ ) in the upper $10 \mathrm{Mm}$ is close to -1 from the equator to $60^{\circ}$ latitude. We aim to measure the temporal variation of the rotational shear over solar cycle 23 and the rising phase of cycle 24 (1996-2015). We used f mode frequency splitting data spanning 1996 to 2011 from the Michelson Doppler Imager (MDI) and 2010 to 2015 from the Helioseismic Magnetic Imager (HMI). In a first for such studies, the f mode frequency splitting data were obtained from 360-day time series. We used the same method as in our previous work for measuring $\mathrm{d} \ln \Omega / \mathrm{d} \ln r$ from the equator to $80^{\circ}$ latitude in the outer $13 \mathrm{Mm}$ of the Sun. Then, we calculated the variation of the gradient at annual cadence relative to the average over 1996 to 2015 . We found the rotational shear at low latitudes $\left(0^{\circ}\right.$ to $30^{\circ}$ ) to vary in-phase with the solar activity, varying by $\sim \pm 10 \%$ over the period 1996 to 2015 . At high latitudes $\left(60^{\circ}\right.$ to $\left.80^{\circ}\right)$, we found rotational shear to vary in anti-phase with the solar activity. By comparing the radial gradient obtained from the splittings of the 360-day and the corresponding 72-day time series of HMI and MDI data, we suggest that the splittings obtained from the 72-day HMI time series suffer from systematic errors. We provide a quantitative measurement of the temporal variation of the outer part of the near surface shear layer which may provide useful constraints on dynamo models and differential rotation theory 1 .
\end{abstract}

\subsection{Introduction}

One of the major challenges in solar physics is to understand the physics behind the 11-year solar cycle. In many dynamo models, which attempt to explain the solar cycle, the

\footnotetext{
1 This section is identical to the published article: "Solar-cycle variation of the rotational shear near the solar surface", A. Barekat, J. Schou, L. Gizon, Astronomy \& Astrophysics 595, A8, 2016, reproduced with permission of Astronomy \& Astrophysics journal (c) ESO. A. Barekat contributed to performing the research, analyzing the data and writing the article.
} 
differential rotation of the Sun plays an important role (see the reviews by Brandenburg and Subramanian (2005) and Charbonneau (2010)).

In an $\alpha \Omega$ dynamo, rotational shear is responsible for the $\Omega$-effect which generates toroidal magnetic field from a poloidal magnetic field. The time variation of the shear has a direct influence on the magnetic field generation in the Sun as it may provide non-linear feedback on the dynamo mechanism (Küker et al. 1999). Additionally, the radial shear in the near-surface shear layer is a potential explanation for the equatorward migration of the activity belt during the solar cycle (Brandenburg 2005). Hence, providing quantitative information about the radial gradient of the rotation close to the surface of the Sun is indispensable. Measurements of the radial shear can also deliver constraints on differential rotation models (e.g., Kitchatinov and Rüdiger 2005). Kitchatinov (2016) recently related the near-surface shear to the subsurface magnetic field. Therefore, the time variation of the shear with the solar cycle may also help estimate the strength of the magnetic field below the surface at different phases of the cycle.

The radial shear can be measured by several helioseismic techniques; see Thompson et al. (1996), Schou et al. (1998), and the latest reviews of global and local helioseismology by Howe (2009) and Gizon et al. (2010), respectively. Corbard and Thompson (2002) showed that the logarithmic radial gradient in the outer $16 \mathrm{Mm}$ of the Sun is close to -1 up to $30^{\circ}$ latitude and becomes positive above $55^{\circ}$ latitude. However, Barekat et al. (2014), hereafter BSG, found no indication of a change of sign at this latitude.

Antia et al. (2008) studied the time variation of the radial and latitudinal shear during solar cycle 23. They used 12 years (1996-2007) of p mode and f mode frequency splitting data from the Michelson Doppler Imager (MDI; Scherrer et al.|1995) on board the Solar and Heliospheric Observatory (SOHO). They also used 13 years (1995-2007) p mode frequency splitting data from the Global Oscillation Network Group (GONG). They applied a two-dimensional regularized least square method (Antia et al. 1998) for inferring the rotation rate. Then, they studied the time variation of both radial and latitudinal shears at several depths and latitudes. They found that the variation of the radial shear is about $20 \%$ of its average value at low latitudes at $14 \mathrm{Mm}$ and below.

In this work, we investigate the solar cycle variation of the radial gradient of the rotation in the outer $13 \mathrm{Mm}$ of the Sun using f modes. We use 19 consecutive years of frequency splitting data corresponding to the entire solar cycle 23 (1996-2010) and the rising phase of cycle 24 (2010-2015). These data are obtained from 360-day time series from the Medium- $l$ program of MDI and from the Helioseismic and Magnetic Imager (HMI; Schou et al. 2012) on board the Solar Dynamics Observatory. These data are different from what we used in BSG in which the splittings were obtained from 72-day time series. Therefore, we compare the gradient obtained from these two different data sets in Section 3.4.1 before we investigate the time variation of the gradient in Section 3.4.2.

\subsection{Observational data}

We consider only f modes. We denote mode frequency by $v_{l m}$ where $l$ and $m$ are the spherical harmonic degree and for azimuthal order, respectively. We use 18 odd $a$-coefficients 
Table 3.1: Summary of 15 years of the MDI and five years (16-20) of the HMI data.

\begin{tabular}{cccccccccccccccc}
\hline data set & starting date & $n$ & $l$ & $n_{1}$ & $l_{1}$ & $n_{2}$ & $l_{2}$ & $n_{3}$ & $l_{3}$ & $n_{4}$ & $l_{4}$ & $n_{5}$ & $l_{5}$ & $n_{\mathrm{c}}$ & $l_{c}$ \\
\hline 1 & 1996.05 .01 & 187 & 98 & 128 & 134 & 123 & 124 & 122 & 142 & 143 & 126 & 132 & 134 & 85 & 180 \\
2 & 1997.04 .26 & 182 & 104 & 120 & 133 & 129 & 135 & 126 & 136 & 128 & 131 & 129 & 121 & 83 & 175 \\
3 & 1998.04 .21 & 168 & 114 & 129 & 125 & 113 & 134 & 128 & 130 & - & - & - & - & - & - \\
4 & 1999.04 .16 & 160 & 111 & 132 & 127 & 134 & 133 & 127 & 135 & 127 & 135 & 128 & 127 & 86 & 164 \\
5 & 2000.04 .10 & 181 & 86 & 133 & 136 & 134 & 123 & 129 & 123 & 132 & 117 & 132 & 121 & 86 & 177 \\
6 & 2001.04 .05 & 177 & 108 & 127 & 126 & 124 & 147 & 133 & 113 & 132 & 127 & 122 & 140 & 76 & 191 \\
7 & 2002.03 .31 & 179 & 87 & 130 & 144 & 121 & 121 & 131 & 137 & 129 & 144 & 130 & 124 & 83 & 181 \\
8 & 2003.03 .26 & 179 & 103 & 125 & 140 & 119 & 144 & 134 & 133 & 116 & 124 & 131 & 111 & 79 & 176 \\
9 & 2004.03 .20 & 178 & 96 & 119 & 138 & 124 & 138 & 126 & 116 & 119 & 146 & 131 & 129 & 70 & 180 \\
10 & 2005.03 .15 & 178 & 106 & 131 & 134 & 129 & 135 & 128 & 127 & 128 & 118 & 124 & 132 & 75 & 180 \\
11 & 2006.03 .10 & 173 & 95 & 132 & 137 & 126 & 134 & 133 & 127 & 123 & 119 & 131 & 130 & 77 & 166 \\
12 & 2007.03 .05 & 177 & 106 & 137 & 130 & 125 & 121 & 130 & 132 & 124 & 139 & 126 & 123 & 80 & 175 \\
13 & 2008.02 .28 & 184 & 96 & 132 & 125 & 135 & 118 & 140 & 120 & 137 & 141 & 123 & 134 & 79 & 174 \\
14 & 2009.02 .22 & 183 & 91 & 140 & 135 & 131 & 122 & 127 & 141 & 126 & 136 & 125 & 118 & 70 & 172 \\
15 & 2010.02 .17 & 163 & 128 & 135 & 134 & 138 & 136 & 126 & 139 & 128 & 133 & 113 & 138 & 84 & 164 \\
16 & 2010.04 .30 & 149 & 131 & 119 & 132 & 115 & 139 & 125 & 123 & 120 & 157 & 109 & 144 & 74 & 173 \\
17 & 2011.04 .25 & 152 & 118 & 119 & 127 & 116 & 135 & 122 & 140 & 120 & 136 & 127 & 133 & 79 & 184 \\
18 & 2012.04 .19 & 152 & 110 & 115 & 144 & 116 & 131 & 117 & 137 & 114 & 130 & 108 & 161 & 70 & 196 \\
19 & 2013.04 .14 & 166 & 100 & 125 & 128 & 118 & 147 & 124 & 140 & 124 & 141 & 118 & 134 & 77 & 171 \\
20 & 2014.04 .09 & 173 & 103 & 119 & 149 & 122 & 149 & 114 & 138 & 126 & 125 & 119 & 142 & 75 & 180 \\
\hline
\end{tabular}

for each $l$ (Schou et al. 1994) obtained from MDI and HMI data, which are defined by

$$
v_{l m}=v_{l}+\sum_{j=1}^{36} a_{l, j} \mathcal{P}_{j}^{(l)}(m),
$$

where $v_{l}$ is the mean multiplet frequency, and $\mathcal{P}_{j}^{(l)}$ are orthogonal polynomials of degree $j$. We use two sets of data of each instrument; the $a$-coefficients which are obtained from 72-day and 360-day time series, resulting in four data sets:

- MDI360: 15 sets obtained from 360-day MDI (1996-2011)

- HMI360: 5 sets obtained from 360-day HMI (2010-2015)

- MDI72: 74 sets obtained from 72-day MDI (1996-2011)

- HMI72: 25 sets obtained from 72-day HMI (2010-2015).

We summarize the number of modes found in each data set in Table 3.1, The differences between the splittings obtained from 360-day and 72-day time series of MDI data were investigated in great detail by Larson and Schou (2015), who also provide further details on the analysis.

The MDI72 and HMI72 are used only for the comparison between the results obtained from these data sets and the corresponding results obtained from data sets MDI360 and 
HMI360. We note here that each 360-day time series is the combination of the five corresponding 72-day ones except for the third data set in Table 3.1, which was made from three non-consecutive 72 day time series (Larson and Schou 2015) because of problems with the SOHO spacecraft.

\subsection{Method}

Our method for measuring the radial gradient is identical to the one used by BSG. We explain our method here succinctly and refer the reader to BSG for detailed explanation. We model the rotation rate as changing linearly with depth

$$
\Omega(r, u)=\Omega_{0}(u)+(1-r) \Omega_{1}(u),
$$

where $r$ is the distance to the center of the Sun normalized by its photospheric radius $\left(R_{\odot}\right)$, $u$ is the cosine of co-latitude and, $\Omega_{0}(u)$ and $\Omega_{1}(u)$ are the rotation rate at the surface and the slope, respectively. Then, we perform a forward problem using the relation between the $a$-coefficients and $\Omega$ which is given by

$$
2 \pi a_{l, 2 s+1}=\int_{0}^{1} \mathrm{~d} r \int_{-1}^{1} \mathrm{~d} u K_{l s}(r, u) \Omega(r, u),
$$

where $K_{l s}$ are kernels. We obtain

$$
\widetilde{\Omega}_{l s} \equiv \frac{2 \pi a_{l, 2 s+1}}{\beta_{l s}}=\left\langle\Omega_{0}\right\rangle_{s}+\left(1-\bar{r}_{l s}\right)\left\langle\Omega_{1}\right\rangle_{s},
$$

where the $\beta_{l s}$ are the total integrals of the radial component of the kernels (see Eq.(4) in BSG) and $\bar{r}_{l s}$ is the central of gravity of the radial kernels. The \langle\rangle denotes latitudinal averages. Next, we perform an error-weighted least square fit of $\widetilde{\Omega}_{l s} / 2 \pi$ versus $\left(1-\bar{r}_{l s}\right)$ to determine $\left\langle\Omega_{0}\right\rangle_{s}$ and $\left\langle\Omega_{1}\right\rangle_{s}$ for each data set.

In the last step of our analysis, we apply the inversion method used by Schou (1999) to $\left\langle\Omega_{0}\right\rangle_{s}$ and $\left\langle\Omega_{1}\right\rangle_{s}$ to infer the rotation rate at each target latitude $u_{0}$ and from this obtain $\mathrm{d} \ln \Omega / \mathrm{d} \ln r$.

\subsection{Results}

Figure 1 shows the radial gradient obtained from the MDI360 and the HMI360 data sets. Also shown in Fig. 3.1 and summarized in Table 3.2 is the value of the 19 year (1996-2015) time average of $\mathrm{d} \ln \Omega / \mathrm{d} \ln r$. Going from the equator, this average fluctuates between -0.97 and -0.9 up to $50^{\circ}$ latitude, above which it steadily increases with latitude. We included data sets 15 and 16 in the average even though they have 288 days of overlap.

Figure 1 also shows consecutive five year time averages of $d \ln \Omega / d \ln r$ which roughly represent different phases of two solar cycles. There is evidence of the solar cycle variation of $\mathrm{d} \ln \Omega / \mathrm{d} \ln r$ at low and high latitudes. These results lead us to investigate the temporal variation of $\mathrm{d} \ln \Omega / \mathrm{d} \ln r$ with annual cadence. We show the results in Section 3.4 .2 .

We note that the time averaged value obtained from the HMI360 data set does not show the same trend as the one measured in BSG above $60^{\circ}$ latitude using the first 20 sets of HMI72 (see Fig. 3.3 of BSG). We explore the difference between our results and BSG of each instrument in detail in the next section. 


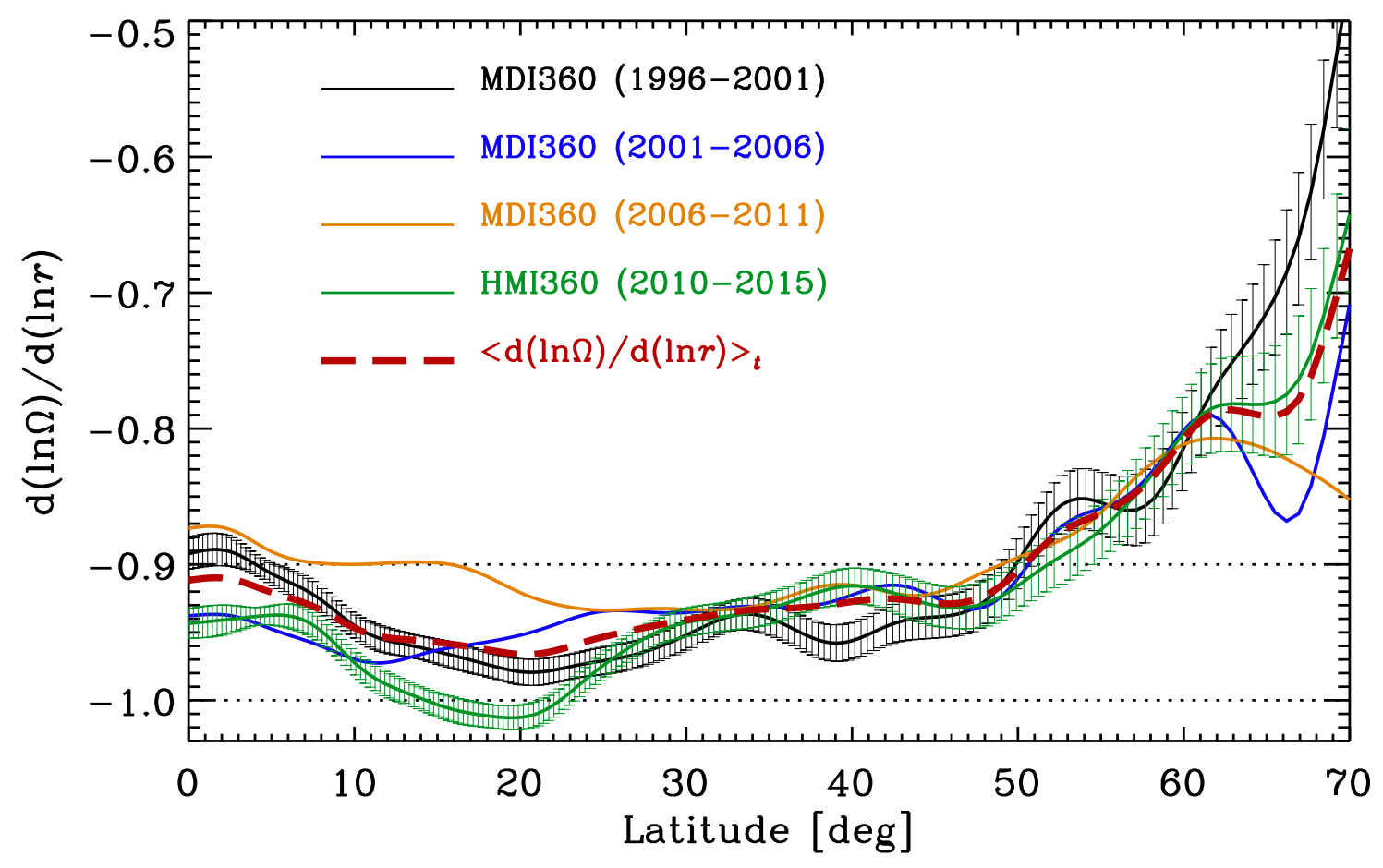

Figure 3.1: Time average of the logarithmic radial gradient versus target latitude. Black, blue, and orange lines represent each consecutive five year time average of $d \ln \Omega / d \ln r$ obtained from MDI360. The green line shows the same quantity obtained from HMI360. The red dashed line shows the 19 year (1996-2015) time average of $d \ln \Omega / d \ln r$. The error bars are $1 \sigma$. The errors on the orange and blue lines are similar to the black one. The errors on the red dashed line are similar to the thickness of the line.

Table 3.2: Selected values of 19 year (1996-2015) time averaged values of the logarithmic radial gradient from Fig. 3.1 .

\begin{tabular}{cc}
\hline \hline Latitude & $<\mathrm{d} \ln \Omega / \mathrm{d} \ln r>_{t}$ \\
\hline $0^{\circ}$ & $-0.912 \pm 0.004$ \\
$10^{\circ}$ & $-0.947 \pm 0.003$ \\
$20^{\circ}$ & $-0.966 \pm 0.004$ \\
$30^{\circ}$ & $-0.941 \pm 0.004$ \\
$40^{\circ}$ & $-0.927 \pm 0.005$ \\
$50^{\circ}$ & $-0.906 \pm 0.007$ \\
$60^{\circ}$ & $-0.809 \pm 0.011$ \\
\hline
\end{tabular}

\subsubsection{Results obtained from 72-day vs. 360-day data}

In this section, we compare the radial gradient derived from splittings obtained from 360-day time series and 72-day time series from both MDI and HMI. First, we show the results of MDI data and then HMI.

The first panel of Fig. 3.2 shows the 15 year (1996-2011) time average of the radial 
gradient obtained from data sets MDI360 and MDI72. The result from data set MDI72 is identical to the MDI result found by BSG. For the MDI data, the absolute value of $\mathrm{d} \ln \Omega / \mathrm{d} \ln r$ is about $5 \%$ smaller than the values found by BSG. This difference can be explained by the fact that using MDI360 and HMI360 data sets enables us to probe roughly $3 \mathrm{Mm}$ deeper than using data sets MDI72 and HMI72. As a consequence, $\widetilde{\Omega}_{l 0} / 2 \pi$ is not linear in $r$ any more, as shown in Fig. 3.3. which in turn means that the fitted values depend on the modes included.

The maximum value of $l=300$ is the same for all data sets, but the minimum value of $l$ is different; see Table 3.1. Therefore, we compare the results obtained from each set in MDI360 with those from the corresponding five sets of MDI72, using only the common modes. The result is shown in the first panel of Fig. 3.2. For this comparison we excluded the last data set of MDI72 because it is after the last set in MDI360. Considering only common modes causes us to exclude more than half of the modes from each data set in MDI360 (see last column of Table 3.1). The difference between the results obtained from MDI360 and MDI72 are reduced substantially and they are now in agreement to better than $1 \sigma$ up to $50^{\circ}$ latitude. This difference increases gradually toward higher latitudes which shows that the results above $50^{\circ}$ latitude are not reliable. We note that one would expect the results to be consistent to better than $1 \sigma$, as they are obtained from the same underlying data. Thus there is clear evidence that the splitting data suffer from systematic errors.

We applied the same comparison to sets HMI360 and HMI72. The five year time averages from using both all and only the common modes are shown in the bottom panel of Fig. 3.2. There is a significant discrepancy between the two results obtained from sets HMI360 and HMI72 above $60^{\circ}$ latitude which does not disappear even when comparing the results obtained from the common modes. This shows that the HMI data are even more affected by systematic errors than the MDI data.

For HMI data, we carry out further analysis by comparing the results derived from common modes of each year. Except for the first and last year the difference between the results persists. The perfect agreement of the results in the last year encourage us to compare common modes between these two data sets. This comparison shows that the difference between $a_{3}$ and $a_{5}$ of those data sets are significant. In average, the values of $a_{3}$ of HMI360 is larger and $a_{5}$ is smaller than the corresponding HMI72 ones by about $3 \sigma$. There are also clear systematic errors in those coefficients with larger discrepancies in the earlier than in the later years.

Unfortunately, these comparisons do not tell us what causes the systematic errors or how to correct them. Understanding this will require a more detailed analysis (Larson \& Schou in prep.). However, our results suggest that HMI72 suffer from systematic errors as the results obtained using HMI360 are not significantly different from the results of data sets MDI360 and MDI72. Moreover, we expect that the splittings obtained from longer time series have better quality as the peaks are better resolved (Larson and Schou 2015).

\subsubsection{Solar cycle variation of the radial gradient}

We measure the variation of $\mathrm{d} \ln \Omega / \mathrm{d} \ln r$ relative to its time averaged value from 1996 to 2015 using data sets MDI360 and HMI360. We show the results in Fig. 3.4 together with the butterfly diagram. These measurements reveal two cyclic patterns; one at low latitudes 

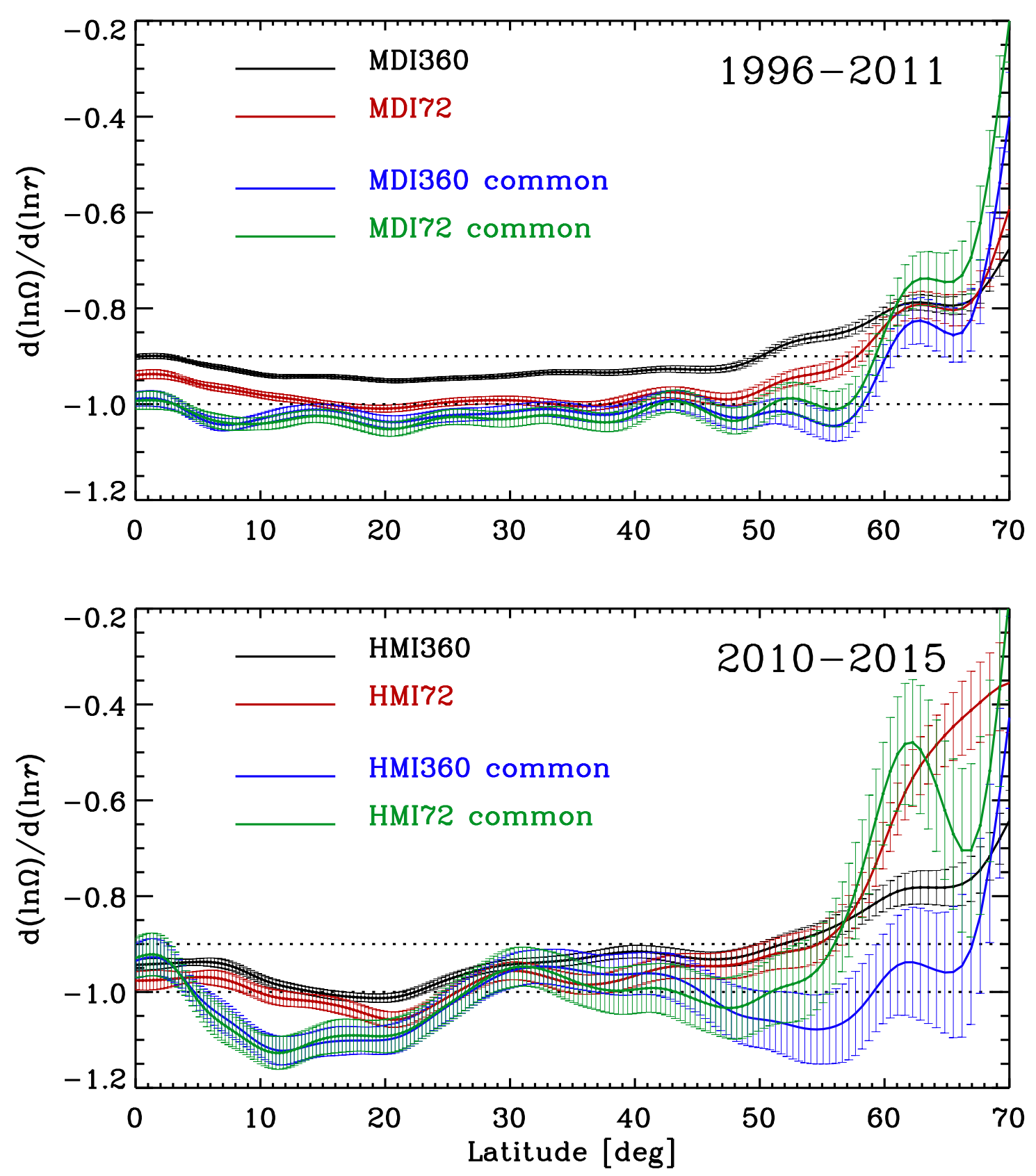

Figure 3.2: Comparison of time averages of $\mathrm{d} \ln \Omega / \mathrm{d} \ln r$ versus target latitude using 15 years of MDI data (upper panel) and five years of HMI data (lower panel). In both panels, black and blue lines show results obtained from splittings from 360-day time series of all and common modes (see, Section 3.4.1), respectively. The red and green lines show the results obtained from 72-day time series of all and common modes, respectively. The dotted lines mark the constant values of -0.9 and -1 at all latitudes. The error bars are $1 \sigma$.

from the equator to about $40^{\circ}$ latitude and one above $60^{\circ}$ latitude. There is no clear signal between about $40^{\circ}$ and $60^{\circ}$ latitude.

Below $40^{\circ}$, there exist bands where the rotation gradient is about $10 \%$ larger and 


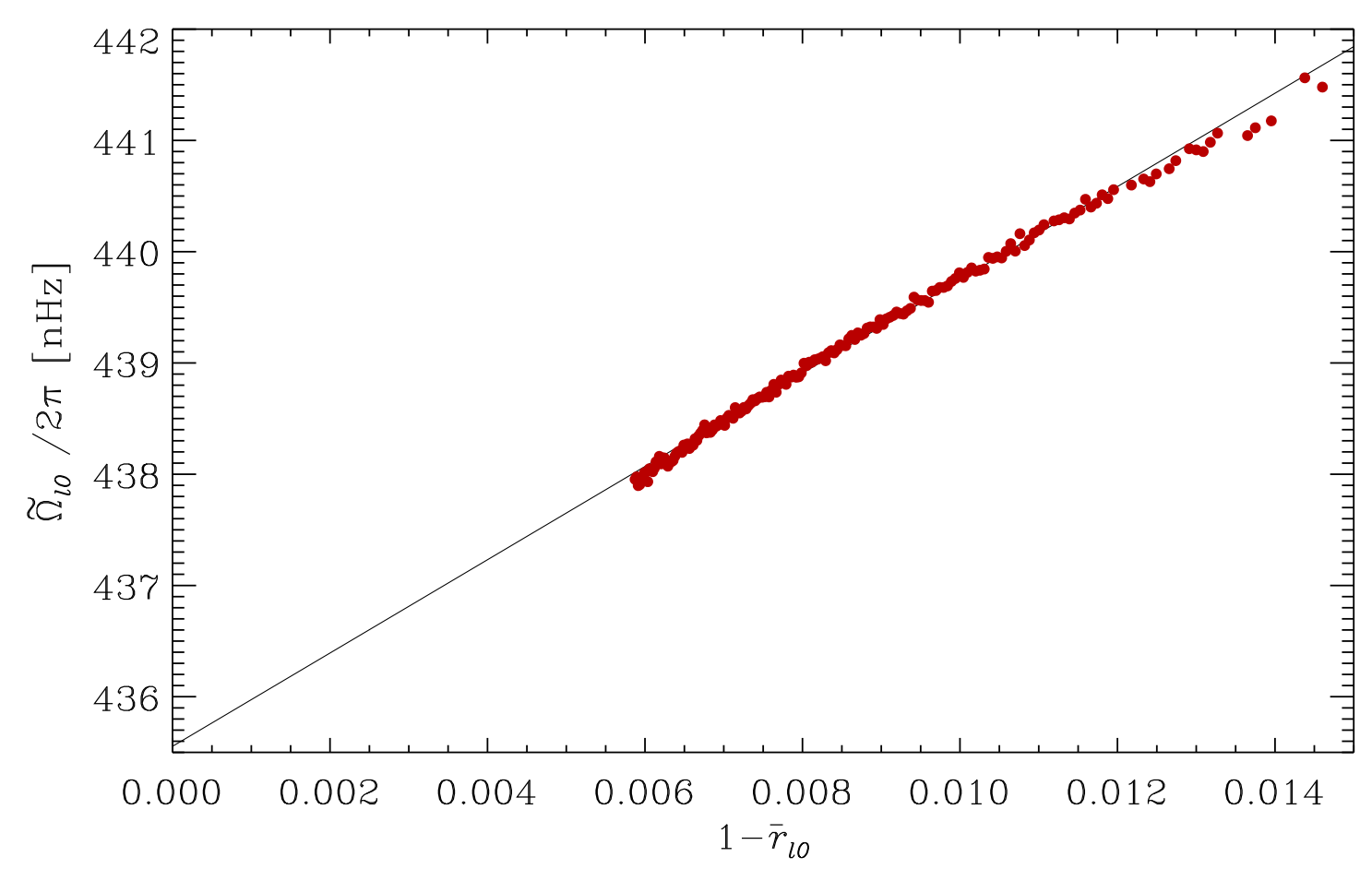

Figure 3.3: $\widetilde{\Omega}_{10} / 2 \pi$ verses $\left(1-\bar{r}_{10}\right)$ obtained from the data set starting 10 April 2000 of MDI360. The black line is the error weighted linear least square fit. We avoid plotting the error bars as they are in similar size of the symbols.

smaller than the average. As illustrated by the butterfly diagram in Figure 4, the band with steeper than average gradient (blue in Fig. 3.4) follows the activity belt quite closely. These bands are also similar to the torsional oscillation signal (see, e.g., Howe et al. (2006), Antia et al. (2008))

The temporal variation of $\mathrm{d} \ln \Omega / \mathrm{d} \ln r$ at high latitudes is more than $10 \%$ of its average value and has the opposite behavior to that at low latitudes. However, as we pointed out eirlier the measured values of the gradient above $50^{\circ}$ latitude are not reliable, so any results here have to be interpreted with caution.

The statistical significance of these signals is shown in Fig. 3.5 and the standard deviation in time and the time averaged errors in Fig. 3.6. The measured signals are statistically significant at low and high latitudes as they are at the 3 to $8 \sigma$ level, while they are indeed not significant between $40^{\circ}$ and $60^{\circ}$ latitude.

We note here that the results obtained from MDI360 and HMI360 are only different by about $1 \%$ when using modes with $l \geqslant 120$, corresponding roughly to the range used by the 72-day analysis and over which the rotation rate changes linearly with depth.

It is well known that the phase and amplitude of the solar cycle variations of the rotation rate vary with depth and latitude (Vorontsov et al.|2002, Basu and Antia|2003, Howe et al. 2005, Antia et al. 2008), but the temporal variation of the gradient has not been previously reported over the same depth range as used in this work.

Antia et al. (2008) found a similar pattern with similar amplitude of the temporal variation of the radial gradient at $0.98 R_{\odot}$ as ours. They used the first eight odd $a$-coefficients 


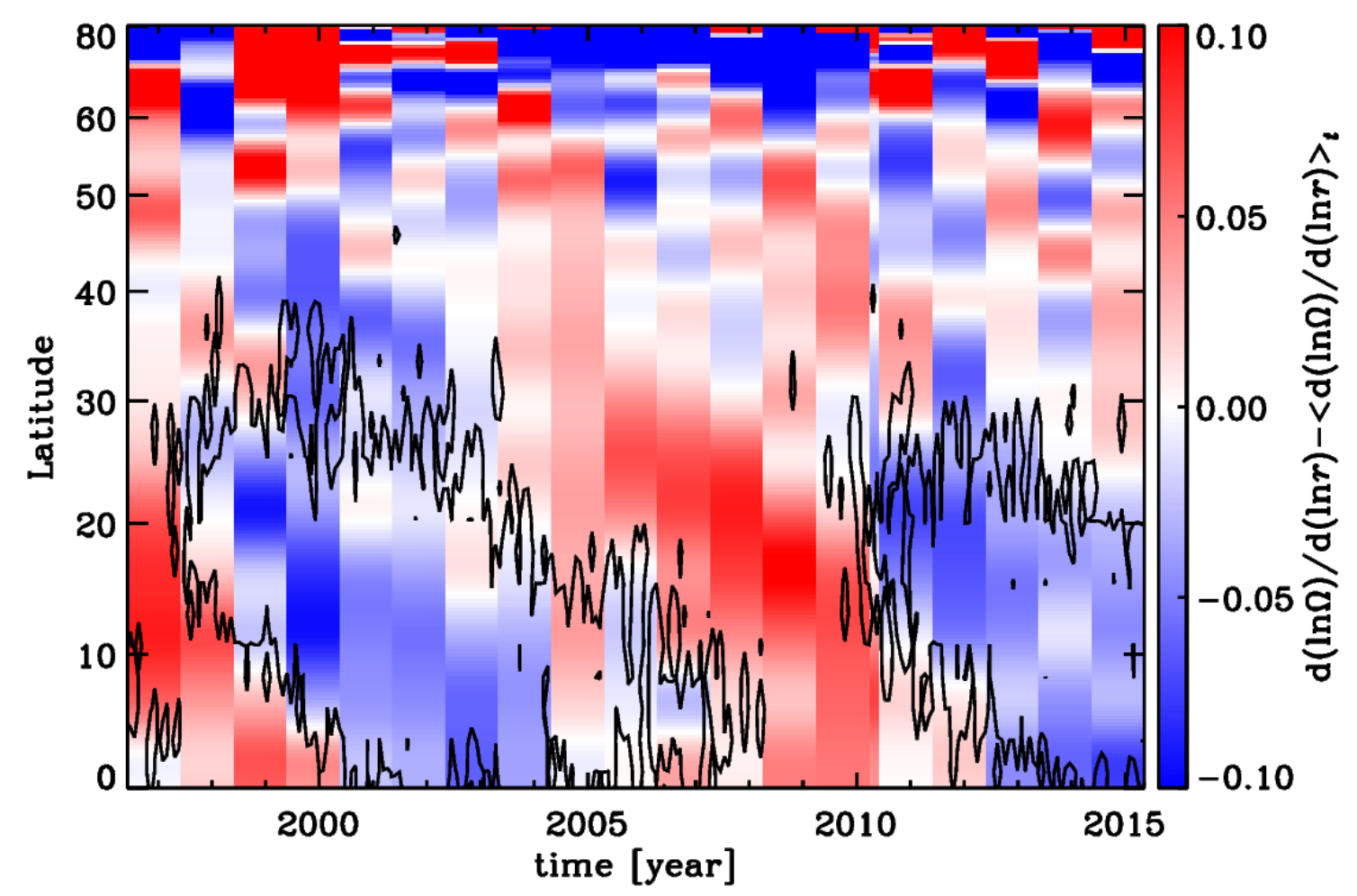

Figure 3.4: Time variation of $\mathrm{d} \ln \Omega / \mathrm{d} \ln r$ relative to its 19 year time average. The thin stripe in the plot shows the result obtained from data set 15 overploted on data set 16 as there is 288 days overlap between these two data sets. The contours show the two hemisphere averaged butterfly diagram of the sunspot area of 5 per millionths of a hemisphere (courtesy of D. Hathaway; see http: //solarscience.msfc.nasa.gov/greenwch. shtml).

obtained from MDI $p$ and f modes and GONG p modes spanning 1995 to 2007. Despite the similarity in pattern and amplitude, the sign of the change in the gradient of their results is opposite to ours. They saw that sunspots occurred where the absolute value of the gradient is smaller than the average value which is the opposite of what we see. This difference might come from the fact that we are measuring the temporal variation around $0.99 R_{\odot}$ while they measured it at $0.98 R_{\odot}$. We also note that Antia et al. (2008) used the earlier version of the MDI data (see Larson and Schou (2015) and BSG) which might explain the discrepancy that Antia et al. (2008) saw between GONG and MDI data at $0.98 R_{\odot}$ and shallower layers.

\subsection{Conclusion}

We make measurements of the radial gradient over 19 years (1996-2015) corresponding to solar cycle 23 and the rising phase of cycle 24 in the outer $13 \mathrm{Mm}$ of the Sun. We use recently available $f$ mode frequency splittings data obtained from 360-day time series of MDI spanning 1996 to 2011 and HMI spanning 2010 to 2015. The values of the radial gradient derived from MDI360 and HMI360 fluctuate between -0.97 and -0.9 up to $50^{\circ}$ latitude. These values are a few percent larger than measured values by BSG which are 


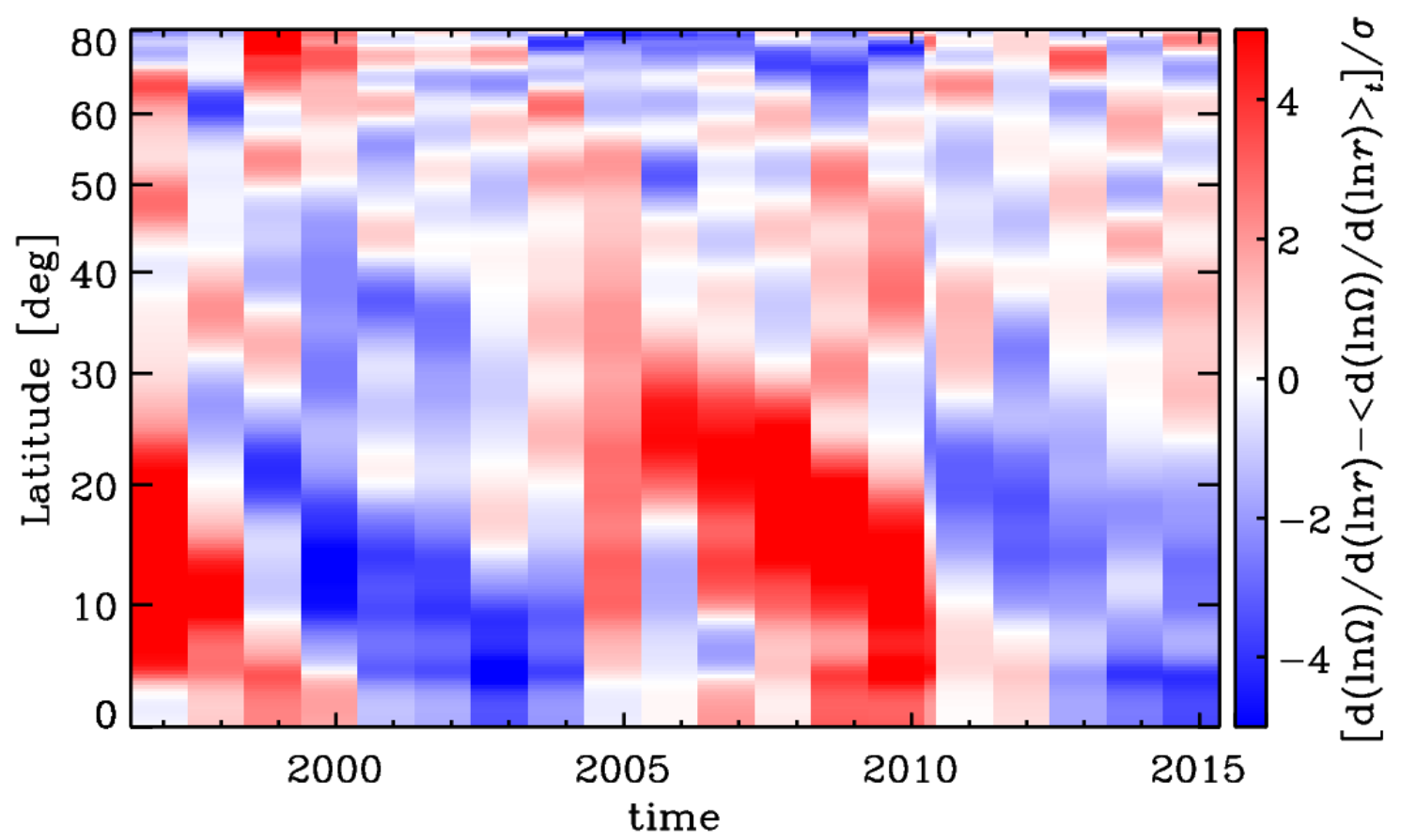

Figure 3.5: Statistical significance in change of $\mathrm{d} \ln \Omega / \mathrm{d} \ln r$ relative to its average values at different latitudes and time. $\sigma$ is the error on $\mathrm{d} \ln \Omega / \mathrm{d} \ln r$ of each year.

obtained from MDI72 and HMI72. It turns out that this difference comes from the fact that the angular velocity does not change linearly with depth to deeper than about $10 \mathrm{Mm}$ below the surface.

We also compare the radial gradient obtained from common modes of two different data sets of each instrument. These comparisons reveal that the measured values of $\mathrm{d} \ln \Omega / \mathrm{d} \ln r$ above $50^{\circ}$ latitude are not reliable. Another important finding is that there are considerable systematic errors in HMI data that needs further investigation.

By measuring the variation of rotational shear relative to its 19 year time averaged value we find two cyclic patterns at low $\left(0^{\circ}\right.$ to $\left.30^{\circ}\right)$ and at high $\left(60^{\circ}\right.$ to $\left.80^{\circ}\right)$ latitudes with similar period of the solar cycle. Both patterns show bands of larger and smaller than average shear moving toward the equator and poles at low and high latitudes, respectively. The relative change in the shear is about $10 \%$ at low latitudes and $20 \%$ at high latitudes. Although the values of $d \ln \Omega / d \ln r$ above $50^{\circ}$ are not reliable, the temporal variation of $d \ln \Omega / d \ln r$ is significant above $60^{\circ}$ latitudes. This finding may have important implications for dynamo models as this variation is considerable compared to the torsional oscillation (Antia et al. 2008).

The cyclic behavior of the shear at low latitudes agrees with the recent theoretical work by Kitchatinov (2016) who showed that the strength of the shear increases because of the presence of the strong magnetic field. Therefore accurate measurements of the shear might be a way of determining of the sub-surface magnetic field. 


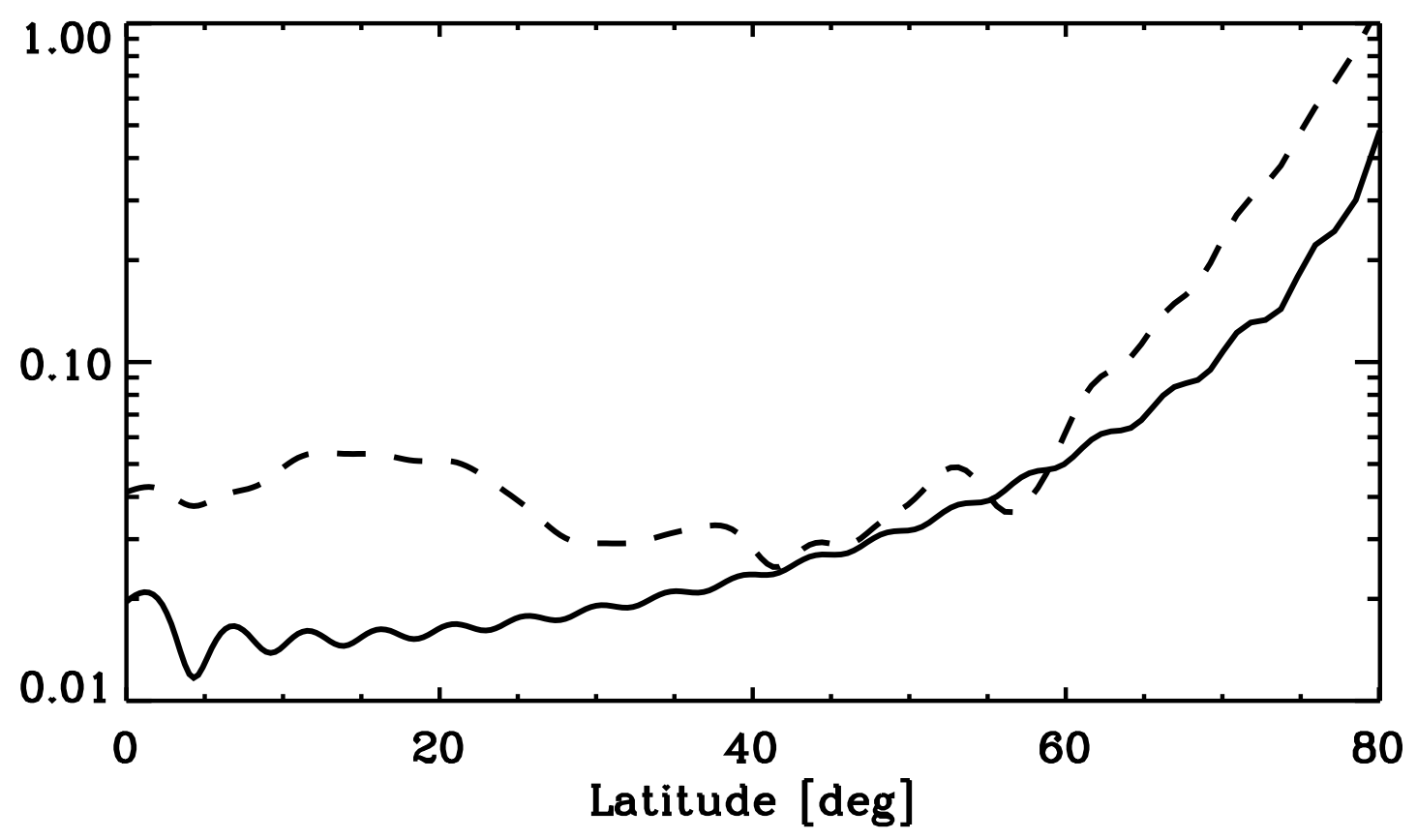

Figure 3.6: Comparison between the standard deviation of the time variation of the radial shear relative to its time averaged value (dashed line) and the time averaged error of the shear (solid line) obtained from data sets MDI360 and HMI360.

\section{Acknowledgements}

We thank T. P. Larson for discussions regarding details of the HMI data, and A. Birch for various discussions and his useful comments about the paper. L. Gizon acknowledges support from the Center for Space Science at the NYU Abu Dhabi Institute. SOHO is a project of international cooperation between ESA and NASA. The HMI data are courtesy of NASA/SDO and the HMI science team. 



\section{Discussion and outlook}

In this thesis, I use $\mathrm{f}$ mode frequency splitting data to measure the radial gradient close to the surface and its variation over solar cycle 23 and 24 relative to its average value. The data used for these measurements come from recently reprocessed 72-day time series of Medium- $l$ MDI and HMI data. Additionally, I use recently available splitting data obtained from both HMI and MDI 360-day time series. The time period of these data is 1 May 1996 to 4 April 2015. The MDI data cover the entire solar cycle $23(\sim 1996-\sim 2010)$ and the HMI data the rising phase of cycle 24 ( 2010 - 2015). The data obtained from 360-day time series probe about $3 \mathrm{Mm}$ deeper than the ones obtained from 72-day time series.

I find that measuring the radial gradient using reprocessed MDI data gives different results from those using the original ones above $30^{\circ}$ latitude reported by Corbard and Thompson (2002). They showed that the gradient is constant and close to -1 from the equator to $30^{\circ}$ and gradually increases from this latitude and become positive above $55^{\circ}$. I show that the value of the logarithmic gradient is $-1 \lesssim \mathrm{d} \ln \Omega / \mathrm{d} \ln r \lesssim-0.9$ up to about $60^{\circ}$. I find that the gradient obtained from HMI 72-day time series not only is significantly different from MDI data, but also from corresponding 360-day time series above $\sim 55^{\circ}$ latitude. This strongly indicates that the HMI data from 72-day time series suffer from systematic errors. The measured values of the time averaged gradient is about $5 \%$ larger using the data obtained from longer time series. Further analysis shows that this is because there are more lower degree modes in the data sets made using longer time series, which can probe deeper layers. This analysis also reveals that the gradient changes linearly with depth only down to about $10 \mathrm{Mm}$ below the surface.

By comparing the gradient with its time averaged values I find two cyclic patterns: one at low and one at high latitudes. The cyclic pattern at low latitudes follows closely the sunspot cycle and the amplitude of its variations is about $\pm 10 \%$ of its time averaged value. Although our results are not reliable above $50^{\circ}$, the time variation of the gradient at those latitudes is significant. The amplitude of the temporal variation of the gradient relative to its average value is similar to what Antia et al. (2008) found at deeper layers. However, they do not agree about the sign such that the shear becomes stronger at solar maximum while Antia et al. (2008) found that the shear becomes weaker. My findings are in agreement with the theoretical explanation of the effect of the magnetic field on the radial shear explained by Kitchatinov (2016).

Recently, several new splitting data sets from both MDI and HMI have become available (Larson and Schou 2018), from now on LS18. I measure the radial gradient using these new data sets and show the results in the next section. 
Table 4.1: Summary of the 15 full-disk MDI data sets. The parameters $n$ and $l_{\min }$ show the number of available modes and the minimum degree that exist in each data set, respectively.

\begin{tabular}{ccc|cc|cc|cc}
\hline data set & starting date & length & $n$ (fd-ap90) & $l_{\min }$ & $n$ (fd-ap83) & $l_{\min }$ & $n$ (vw-ap83) & $l_{\min }$ \\
\hline 1 & 1996.05 .23 & 63 & 130 & 108 & 122 & 147 & 116 & 151 \\
2 & 1997.04 .13 & 93 & 143 & 123 & 147 & 120 & 144 & 129 \\
3 & 1998.01 .09 & 92 & 142 & 133 & 138 & 133 & 139 & 138 \\
4 & 1999.03 .13 & 77 & 131 & 137 & 128 & 137 & 127 & 149 \\
5 & 2000.05 .27 & 98 & 147 & 122 & 141 & 129 & 143 & 122 \\
6 & 2001.02 .28 & 90 & 145 & 131 & 143 & 127 & 137 & 127 \\
7 & 2002.02 .14 & 109 & 147 & 126 & 146 & 106 & 145 & 137 \\
8 & 2003.09 .10 & 76 & 121 & 140 & 119 & 133 & 115 & 141 \\
9 & 2004.07 .04 & 65 & 126 & 132 & 128 & 139 & 130 & 139 \\
10 & 2005.06 .25 & 67 & 127 & 137 & 130 & 137 & 126 & 116 \\
11 & 2006.03 .24 & 62 & 122 & 146 & 117 & 134 & 120 & 124 \\
12 & 2007.11 .08 & 58 & 117 & 143 & 118 & 111 & 113 & 111 \\
13 & 2008.03 .03 & 64 & 132 & 143 & 132 & 125 & 122 & 125 \\
14 & 2009.05 .18 & 65 & 118 & 126 & 122 & 125 & 122 & 125 \\
15 & 2010.05 .07 & 67 & 125 & 131 & 127 & 124 & 119 & 124 \\
\hline
\end{tabular}

\subsection{Comparison with various other data sets}

LS18 obtained the mode parameters of the oscillation of the Sun using a slightly different processing than that used for the Medium- $l$ program as they use full-disk MDI Doppler images. They also obtain mode parameters using HMI data by applying the Medium- $l$ processing to 72-day time series. First, I will analyze the new MDI data and then HMI.

\subsubsection{Radial gradient obtained from full-disk MDI data}

There are 15 roughly yearly discontinuous full-resolution full-disk MDI data sets that were recently processed by LS18 and cover about $20 \%$ of the MDI mission. The starting date and the length of each data set are listed in Table 4.1. The lengths of these time series vary between 58 to 109 days and half of the data sets have a shorter length than 72 days. The details of processing and obtaining the mode parameters of these new data sets are explained in LS18.

The major differences between these ones and the Medium- $l$ data are that these data were transmitted as full disk images and were not convolved with any kernels on board. More importantly, for the analysis used to obtain the mode parameters these data are apodized between $0.9 R_{\odot}$ and $0.95 R_{\odot}$ instead of $0.83 R_{\odot}$ and $0.87 R_{\odot}$ as was done for the Medium- $l$ data. In order to make an easier comparison between the mode parameters obtained from Medium- $l$ and full-disk data, LS18 also applied Medium- $l$ analysis on the full-disk MDI data. Moreover, they used full-disk data and apodize them similar to Medium- $l$ to study only the effect of the different apodization on the data. They showed that using a different apodization radius causes significant differences between the mode parameters and that a systematic error that they were struggling for a decade to remove has 
disappeared (Schou 1992, Schou et al. 2002).

In summary I am going to use three data sets naming them identical to LS18 as follows:

- fd-ap90: 15 full-disk data sets apodized between $0.9 R_{\odot}$ and $0.95 R_{\odot}$

- fd-ap83: 15 full-disk data sets apodized between $0.83 R_{\odot}$ and $0.87 R_{\odot}$

- vw-ap83: 15 full-disk data sets of Medium- $l$ proxy.

The maximum value of $l=300$ is the same for all data sets, but the mode coverage and the minimum value of $l$ are different as shown in Table 4.1

I measured the time averaged value of $\mathrm{d} \ln \Omega / \mathrm{d} \ln r$ using the three new data sets. The results are shown in Fig. 4.1a and at selected latitudes in Table 4.2. The values of the gradient are similar below about $50^{\circ}$, but they are significantly different at high latitudes as shown in Fig. 4.1 d. In Table 4.2, one can clearly see that the values of the gradient obtained from fd-ap83 are closer to the ones of vw-ap83 than fd-ap90. These results confirm the important findings of LS18 that different apodization of the Doppler images can lead to different results and that the binning of the data has less effect than the apodization.

As the disagreement between the results could be because of the different mode coverage of each data set, I repeat the analysis using only the yearly common modes among all three data sets. The results are shown in Fig. 4.1 p. Considering only common modes did not affect the results significantly. However, the results of fd-ap83 and vw-ap83 show slightly better agreement than using all modes, see Fig. 4.1. I I also repeat the analysis of the common modes using the error on vw-ap83 for the two other data sets. This did not make any significant differences in the results.

A comparison of the $a$-coefficients of these three data sets show that the significant discrepancy between the $a$-coefficients occur at $l \geq 280$. Therefore, I obtained the gradient using common modes excluding $l \geq 280$. The results are shown in Fig. 4.1c. Excluding those high degree modes reduces the differences between the results using the fd-ap90 and those from the other two data sets. Although the results of $l \geq 280$ modes got into better agreement, I cannot confidently conclude that the differences in apodization only affect the high degree modes as the results are still significantly different around $55^{\circ}$.

Table 4.2: 15 years (1996-2010) time averaged value $\mathrm{d} \ln \Omega / \mathrm{d} \ln r$ at selected latitudes obtained from full-disk MDI data.

\begin{tabular}{cccc}
\hline \hline Latitude $[\mathrm{deg}]$ & fd-ap90 & fd-ap83 & vw-ap83 \\
\hline 0 & $-0.944 \pm 0.022$ & $-0.941 \pm 0.022$ & $-0.909 \pm 0.022$ \\
10 & $-0.988 \pm 0.017$ & $-0.979 \pm 0.017$ & $-0.995 \pm 0.016$ \\
20 & $-0.984 \pm 0.019$ & $-1.000 \pm 0.018$ & $-0.995 \pm 0.018$ \\
30 & $-0.940 \pm 0.022$ & $-0.956 \pm 0.022$ & $-0.962 \pm 0.022$ \\
40 & $-0.976 \pm 0.027$ & $-1.012 \pm 0.027$ & $-1.003 \pm 0.027$ \\
50 & $-0.881 \pm 0.036$ & $-0.962 \pm 0.036$ & $-0.920 \pm 0.036$ \\
60 & $-0.867 \pm 0.057$ & $-0.858 \pm 0.056$ & $-0.980 \pm 0.056$ \\
70 & $-0.657 \pm 0.123$ & $-0.232 \pm 0.121$ & $-0.292 \pm 0.121$ \\
\hline
\end{tabular}



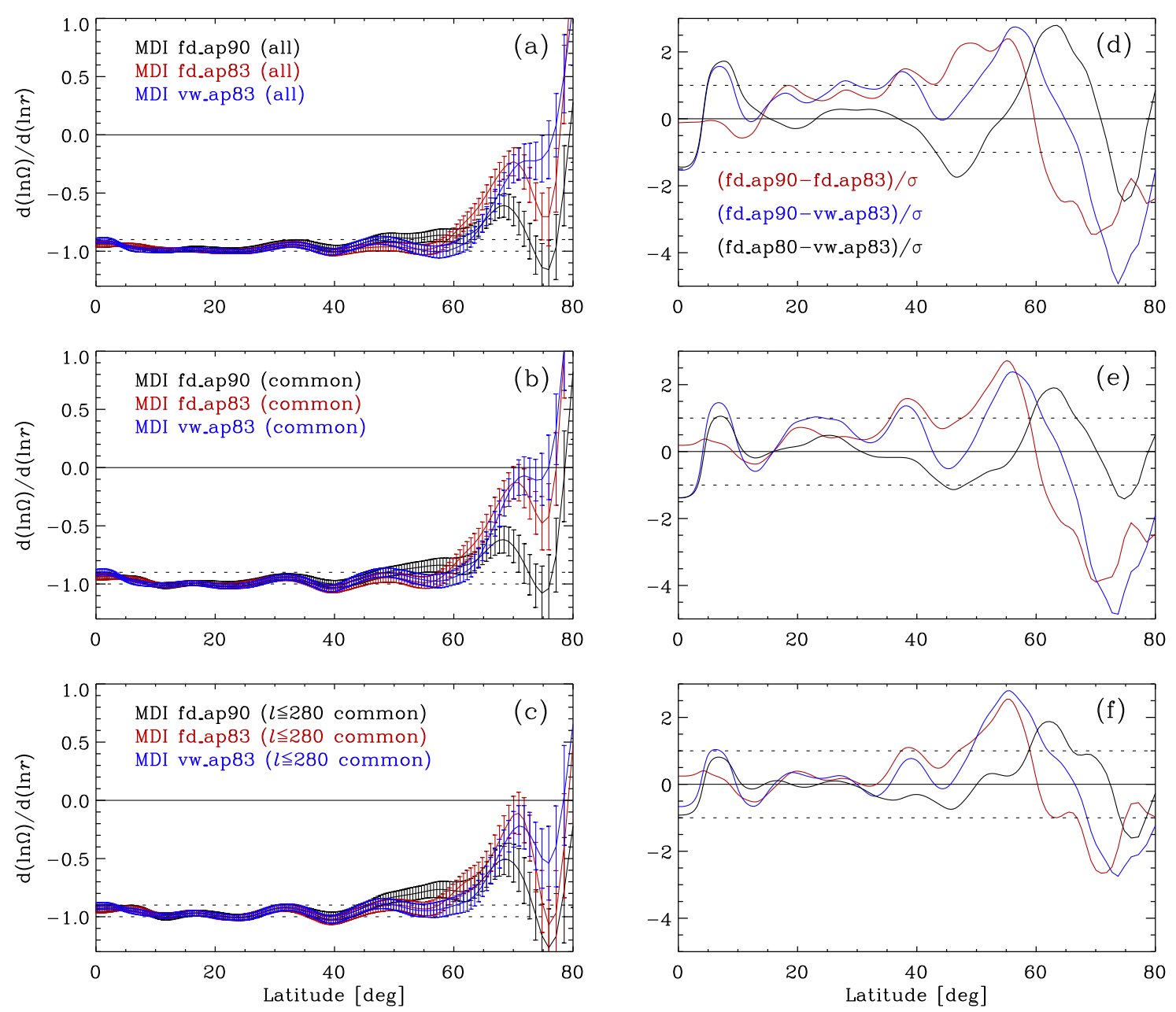

Figure 4.1: Time averaged radial gradient versus latitude obtained from 15 full-disk MDI data sets (left panels) and the significance of the differences (right panels). Panel a) shows the results obtained from all modes, b) from common modes and c) common modes with $l \leq 280$. Black, red and blue lines in these panels show the results obtained from fd-ap90, fd-ap83 and vw-ap83, respectively. The dotted lines mark the constant values of -0.9 and -1 at all latitudes. The error bars are $1 \sigma$. Panel d-f show the corresponding significance of the differences between the results shown in the panel a-c as follow: (fd-ap90-fdap83) $/ \sigma_{\text {fd-ap } 90}\left(\right.$ red), (fd-ap90-vw-ap83) $/ \sigma_{\text {fd-ap90 }}\left(\right.$ blue) and (fd-ap83-vw-ap83) $/ \sigma_{\text {fd-ap83 }}$ (black). The dotted lines mark $\pm 1 \sigma$.

From analyzing all these new data sets I could confirm our previous findings: the value of the radial gradient is not reliable above $50^{\circ}$ and the time averaged value of the gradient is between $-1 \lesssim \mathrm{d} \ln \Omega / \mathrm{d} \ln r \lesssim-0.9$ below $50^{\circ}$. I also note here that the similarity of the differences between the results obtained from fd-ap90 and two other data sets confirm the finding of LS18 which was that the binning of the data has less effect than apodization in obtaining the mode parameters. 


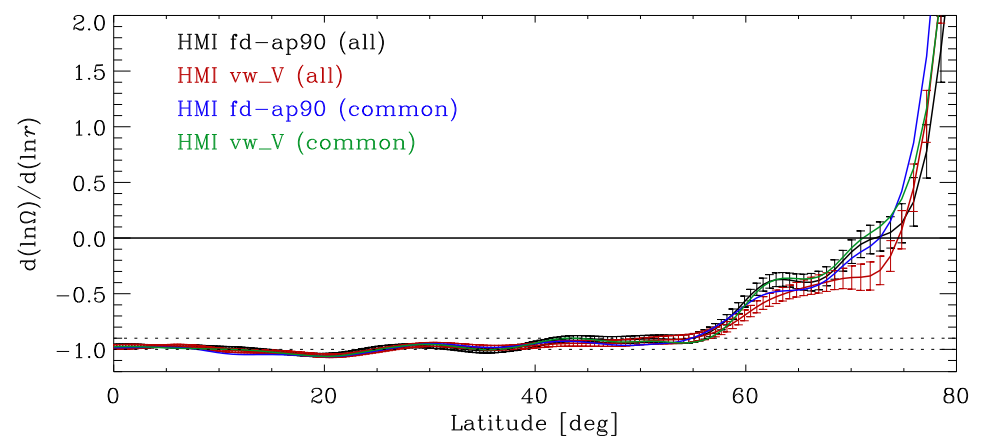

Figure 4.2: Time average (2010-2015) of the radial gradient versus latitude. Black and red lines show the results obtained form full-disk and vw_V HMI data using all available modes, respectively. The results obtained from common modes between the two data sets are shown in blue (full-disk) and green (vw_V) lines. The error bars on the results of using common modes of full-disk (blue) and vw_V (green) are not shown, but they have similar magnitude to those of using all modes. The dotted lines mark the constant values of -0.9 and -1 at all latitudes. The error bars are $1 \sigma$.

\subsubsection{Radial gradient obtained from Medium- $l$ HMI data}

LS18 also obtained frequency splitting data from 72-day HMI time series using the MDI Medium- $l$ proxy. The purpose of producing these data sets was to investigate the effect of the apodization and subsampling of the Doppler images used for obtaining the mode parameters from the HMI data. Their investigation showed that the mode parameters obtained from full-disk and vw_V differ in a manner similar to the corresponding MDI data. Although they investigated these differences in detail, they could not find any clear explanation for all these differences. As a consequence, it is still not possible to determine with certainty which data set is the best one or suffers least from systematic errors.

I distinguish these data sets from the full-disk data used in the last two sections by naming them vw_V similar to LS18. These data provide an excellent opportunity to have a better comparison between the gradient obtained from five sets of simultaneous observations of HMI and MDI than was done in Section 2. Before performing these comparisons I also measured the radial gradient obtained from 25 of these sets spanning 30 April 2010 to 4 April 2015 with identical time spans to those used in Section 3 .

The resulting time averaged gradients using vw_V and full-disk HMI data are shown in Fig. 4.2. This comparison shows that the gradient does not change significantly using Medium- $l$ HMI data sets at low latitudes. In particular, at low latitudes the gradient is close to the reported value of -1 . However, the differences of the results are more than $1 \sigma$ at high latitudes. As the number of modes are not the same for simultaneous data, I perform the analysis using common modes between the two sets, the results of which are shown in Fig. 4.2. The differences reach $1 \sigma$ using common modes at high latitudes. I note here that the discrepancy between some individual data sets reach $4 \sigma$ at high latitudes and $3 \sigma$ at low latitudes. It is not simple to find out what the sources of all the discrepancies are. In the case of HMI data this is disappointing as the full disk HMI data obtained from the different length time series also do not agree. 

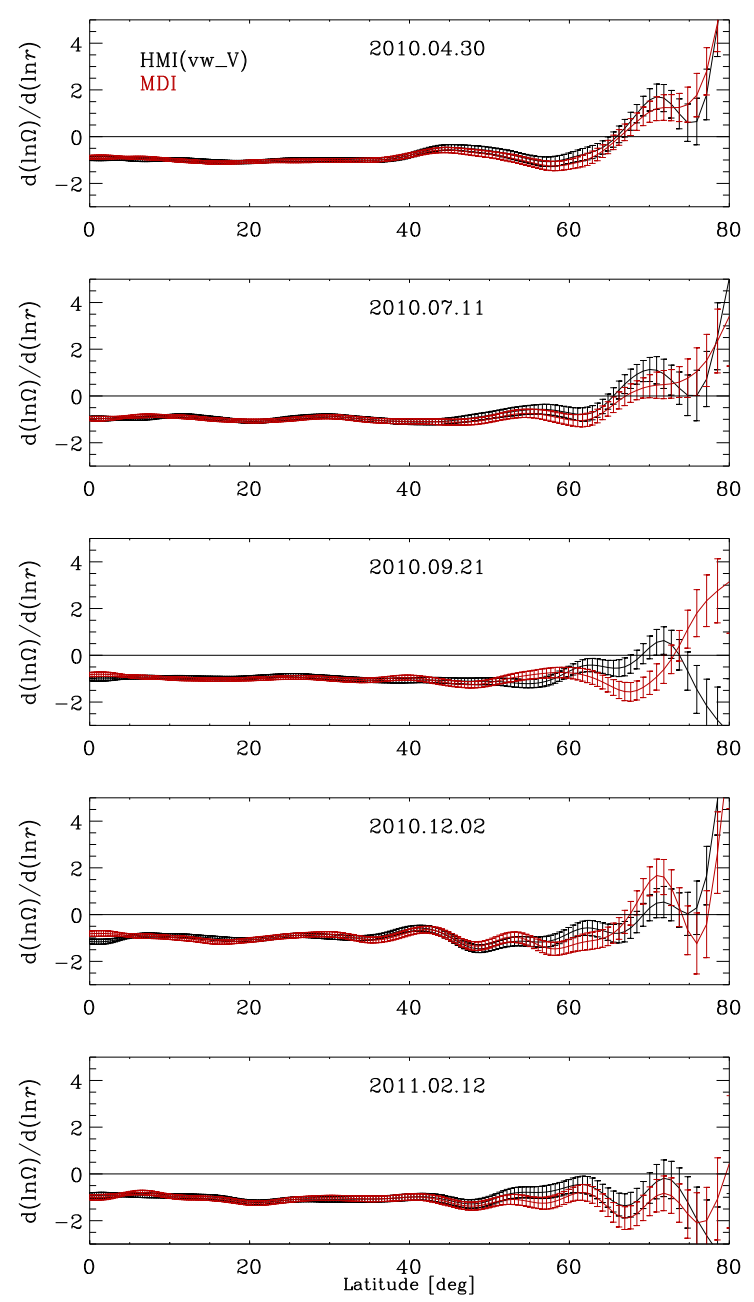

Figure 4.3: Comparison of the radial gradient obtained from common modes between each simultaneous vw_V HMI (black line) and Medium- $l$ MDI (red line) data. The starting date of each pair is indicated in each panel.

I show the results of comparing the gradient obtained from common modes between each simultaneous vw_V HMI and Medium- $l$ MDI data in Fig. 4.3. This comparison shows that the existing discrepancy between the results of the two instruments that were shown in Fig. 2.3 persists even using Medium- $l$ proxy for HMI data. This is not so surprising as both frequencies and $a$-coefficients obtained from these two data sets are significantly different for some $f$ modes as shown in Fig. 16. of LS18.

\subsection{Theoretical implications}

The mechanism behind the formation of the NSSL and its dynamics is not well understood. However, there are several proposed explanations for the formation of the NSSL (e.g. Kitchatinov and Rüdiger 2005, Miesch and Hindman 2011). Therefore, my measurements of the strength of the radial shear and the finding that the gradient does not depend 
on latitude up to $50^{\circ}$, provide strong constraints on these theories. For example, my measurements are in good agreement with the model of Kitchatinov and Rüdiger (2005) who predicted a latitude independent value of $\mathrm{d} \ln \Omega / \mathrm{d} \ln r \approx-0.92$.

Kitchatinov (2016) used the results obtained in Section 2 and developed the model of Kitchatinov and Rüdiger (2005) further by taking into account the effect of the magnetic field. He showed that the presence of a magnetic field can lead to a deviation from a constant value of the gradient at all latitudes. The gradient becomes steeper at the location of strong magnetic field. This causes a weak latitudinal as well as cyclic dependency of gradient. This is indeed in agreement with my results in Section 3 in which I found a weak latitudinal dependency (Maximum shear at $20^{\circ}$ latitude) and a strong gradient during the solar cycle. As suggested by Kitchatinov (2016), we may be able to estimate the subsurface magnetic field by measuring the change in the radial gradient during the solar cycle.

\subsection{Outlook}

In this thesis, I measured the radial gradient of the rotation rate in the outer $13 \mathrm{Mm}$ of the NSSL. In order to have a better understanding of the dynamics of the NSSL we need to measure the gradient of the entire NSSL which extends to $35 \mathrm{Mm}$ below the surface. For such measurements the $\mathrm{p}$ modes should be considered, as these modes probe deeper layers. Unfortunately, our method cannot be applied for those layers as the analysis of $p$ modes shows a significant deviation of the rotation rate from a linear change with depth. Therefore, a different strategy needs to be used to measure the radial gradient in deeper layers.

The layers that I consider for measuring the gradient are also accessible using local helioseismology methods (Gizon et al.2010). This provides a possibility for measuring the radial gradient using other helioseismology techniques and compare our results with the results obtained from those. Such a comparison is encouraging as Howe et al. (2006) showed that there is a discrepancy between the measured gradient obtained by Corbard and Thompson (2002) and the gradient obtained form the ring diagram analysis (Hill 1988) at above $30^{\circ}$ latitude. I note here that this is exactly the latitude above which I show that the gradient is different from measured values of Corbard and Thompson (2002).

One of the major goals of measuring the radial gradient and its cycle variations close to the surface was to have a better understanding of the dynamics of the subsurface layers. However, there are several other phenomenon observed at the surface of the Sun which are not well understood and may have a relation to the measured gradient. One of them is the large scale convective pattern known as supergranulation. These convective patterns have a diameter of about $30 \mathrm{Mm}$ with an average velocity of about $400 \mathrm{~m} / \mathrm{s}$ and a life time of about 1-2 days. These cells can be clearly seen in the Doppler image shown in Fig. 1.4. Supergranulation also shows the fastest rotation rate measured using feature tracking method, see Fig. 1.7. For further details about the supergranulation I refer the reader to the review by Rieutord and Rincon (2010). Gizon et al. (2003) and Schou (2003) studied supergranulation using two different techniques and explained their super rotation by suggesting that they have a wave-like behavior. They showed that the dispersion relation of supergranular wave is independent of direction and latitude. There may also be a physical relation between the radial gradient and the properties of the supergranulation. 
There is an ongoing investigation related to the discrepancy between HMI 72-day and 360-day time series (private communication with T. Larson). The goal of this investigation is to find the cause of these discrepancies and hopefully removing the systematic errors. Having better quality data may help to have a better measurements of the radial gradient in high latitudes in the near future.

Furthermore, there is a planned space mission called the "Solar Orbiter" which will observe the Sun with an inclined orbit relative to the ecliptic plane (Müller et al. 2013). This will provide the possibility of observing the poles of the Sun. The Polarimetric and Helioseismic Imager (PHI) instrument on board of the Solar Orbiter will provide Doppler images using $6173 \AA$ FeI absorption line with a cadence of 60 seconds. Unfortunately, these data cannot be used for global modes analysis because of the short duration of the observation, but still can be used for local helioseismology analysis (Löptien et al. 2015). Therefore, only the properties of the shallow layers can be extracted from these data. These observations will provide a great opportunity to measure the rotation rate and its radial gradient at latitudes which could not been determined with the currently available data sets. 


\section{Bibliography}

Aerts, C., Christensen-Dalsgaard, J., Kurtz, D. W., 2010, Asteroseismology, Springer

Antia, H. M., Basu, S., 2000, Temporal Variations of the Rotation Rate in the Solar Interior, ApJ, 541, 442-448, astro-ph/0004335

Antia, H. M., Basu, S., Chitre, S. M., 1998, Solar internal rotation rate and the latitudinal variation of the tachocline, MNRAS, 298, 543-556, astro-ph/9709083

Antia, H. M., Basu, S., Chitre, S. M., 2008, Solar Rotation Rate and Its Gradients During Cycle 23, ApJ, 681, 680-692, 0803.3619

Barekat, A., Schou, J., Gizon, L., 2014, The radial gradient of the near-surface shear layer of the Sun, A\&A, 570, L12, 1410.3162

Barekat, A., Schou, J., Gizon, L., 2016, Solar-cycle variation of the rotational shear near the solar surface, A\&A, 595, A8, 1608.07101

Basu, S., Antia, H. M., 2003, Changes in Solar Dynamics from 1995 to 2002, ApJ, 585, 553-565, astro-ph/0211548

Basu, S., Antia, H. M., Tripathy, S. C., 1999, Ring Diagram Analysis of Near-Surface Flows in the Sun, ApJ, 512, 458-470, astro-ph/9809309

Beck, J. G., 2000, A comparison of differential rotation measurements - (Invited Review), Sol. Phys., 191, 47-70

Brandenburg, A., 2005, The Case for a Distributed Solar Dynamo Shaped by Near-Surface Shear, ApJ, 625, 539-547, astro-ph/0502275

Brandenburg, A., Subramanian, K., 2005, Astrophysical magnetic fields and nonlinear dynamo theory, Phys. Rep., 417, 1-209, astro-ph/0405052

Brown, T. M., Christensen-Dalsgaard, J., Dziembowski, W. A., Goode, P., Gough, D. O., Morrow, C. A., 1989, Inferring the sun's internal angular velocity from observed p-mode frequency splittings, ApJ, 343, 526-546

Charbonneau, P., 2010, Dynamo Models of the Solar Cycle, Living Reviews in Solar Physics, 7, 3

Christensen-Dalsgaard, J., Schou, J., 1988, Differential rotation in the solar interior, in Seismology of the Sun and Sun-Like Stars, (Ed.) E. J. Rolfe, vol. 286 of ESA Special Publication, pp. 149-153 
Christensen-Dalsgaard, J., Schou, J., Thompson, M. J., 1990, A comparison of methods for inverting helioseismic data, MNRAS, 242, 353-369

Corbard, T., Thompson, M. J., 2002, The subsurface radial gradient of solar angular velocity from MDI f-mode observations, Sol. Phys., 205, 211-229 (CT), astro-ph/0110361

Cox, J. P., 1980, Theory of stellar pulsation

Delury, R. E., 1939, The Law of Solar Rotation (with Plate XIII), JRASC, 33, 345

Deubner, F.-L., 1975, Observations of low wavenumber nonradial eigenmodes of the sun, A\&A, 44, 371-375

Dikpati, M., Charbonneau, P., 1999, A Babcock-Leighton Flux Transport Dynamo with Solar-like Differential Rotation, ApJ, 518, 508-520

Dikpati, M., Corbard, T., Thompson, M. J., Gilman, P. A., 2002, Flux Transport Solar Dynamos with Near-Surface Radial Shear, ApJ, 575, L41-L45

Foukal, P., 1977, Supergranulation and the dynamics of gas and magnetic field below the solar photosphere, ApJ, 218, 539-546

Giles, P. M., Duvall, Jr., T. L., Scherrer, P. H., 1998, Time-Distance Measurements of Subsurface Rotation and Meridional Flow, in Structure and Dynamics of the Interior of the Sun and Sun-like Stars, (Ed.) S. Korzennik, vol. 418 of ESA Special Publication, p. 775

Gilman, P. A., 1974, Solar rotation, ARA\&A, 12, 47-70

Gilman, P. A., Foukal, P. V., 1979, Angular velocity gradients in the solar convection zone, ApJ, 229, 1179-1185

Gizon, L., Duvall, T. L., Schou, J., 2003, Wave-like properties of solar supergranulation, Nature, 421, 43-44, astro-ph/0208343

Gizon, L., Birch, A. C., Spruit, H. C., 2010, Local Helioseismology: Three-Dimensional Imaging of the Solar Interior, ARA\&A, 48, 289-338, 1001.0930

Gough, D. O., 1993, Linear adiabatic stellar pulsation., in Astrophysical Fluid Dynamics Les Houches 1987, (Eds.) J.-P. Zahn, J. Zinn-Justin, pp. 399-560

Guerrero, G., Smolarkiewicz, P. K., Kosovichev, A. G., Mansour, N. N., 2013, Differential Rotation in Solar-like Stars from Global Simulations, ApJ, 779, 176, 1310.8178

Hale, G. E., 1908, On the Probable Existence of a Magnetic Field in Sun-Spots, ApJ, 28, 315

Harvey, J. W., Hill, F., Hubbard, R. P., Kennedy, J. R., Leibacher, J. W., Pintar, J. A., Gilman, P. A., Noyes, R. W., Title, A. M., Toomre, J., Ulrich, R. K., Bhatnagar, A., Kennewell, J. A., Marquette, W., Patron, J., Saa, O., Yasukawa, E., 1996, The Global Oscillation Network Group (GONG) Project, Science, 272, 1284-1286 
Hill, F., 1988, Rings and trumpets - Three-dimensional power spectra of solar oscillations, ApJ, 333, 996-1013

Howard, R., Harvey, J., 1970, Spectroscopic Determinations of Solar Rotation, Sol. Phys., $12,23-51$

Howard, R., Labonte, B. J., 1980, The sun is observed to be a torsional oscillator with a period of 11 years, ApJ, 239, L33-L36

Howe, R., 2009, Solar Interior Rotation and its Variation, Living Reviews in Solar Physics, $6,0902.2406$

Howe, R., Christensen-Dalsgaard, J., Hill, F., Komm, R. W., Larsen, R. M., Schou, J., Thompson, M. J., Toomre, J., 2000a, Deeply Penetrating Banded Zonal Flows in the Solar Convection Zone, ApJ, 533, L163-L166, astro-ph/0003121

Howe, R., Christensen-Dalsgaard, J., Hill, F., Komm, R. W., Larsen, R. M., Schou, J., Thompson, M. J., Toomre, J., 2000b, Dynamic Variations at the Base of the Solar Convection Zone, Science, 287, 2456-2460

Howe, R., Komm, R., Hill, F., 2000c, Variations in solar sub-surface rotation from GONG data 1995-1998, Sol. Phys., 192, 427-435

Howe, R., Christensen-Dalsgaard, J., Hill, F., Komm, R., Schou, J., Thompson, M. J., 2005, Solar Convection-Zone Dynamics, 1995-2004, ApJ, 634, 1405-1415

Howe, R., Komm, R., Hill, F., Ulrich, R., Haber, D. A., Hindman, B. W., Schou, J., Thompson, M. J., 2006, Large-Scale Zonal Flows Near the Solar Surface, Sol. Phys., $235,1-15$

Hughes, D. W., Rosner, R., Weiss, N. O. (Eds.), 2007, The Solar Tachocline

Käpylä, P. J., Korpi, M. J., Tuominen, 2006, Solar dynamo models with $\alpha$-effect and turbulent pumping from local 3D convection calculations, Astronomische Nachrichten, 327,884 , astro-ph/0606089

Karak, B. B., Cameron, R., 2016, Babcock-Leighton Solar Dynamo: The Role of Downward Pumping and the Equatorward Propagation of Activity, ApJ, 832, 94, 1605.06224

Kichatinov, L. L., Rüdiger, G., 1993, A-effect and differential rotation in stellar convection zones, A\&A, 276, 96

Kitchatinov, L. L., 2013, Theory of differential rotation and meridional circulation, in IAU Symposium, (Eds.) A. G. Kosovichev, E. de Gouveia Dal Pino, Y. Yan, vol. 294 of IAU Symposium, pp. 399-410, 1210.7041

Kitchatinov, L. L., 2016, Rotational shear near the solar surface as a probe for subphotospheric magnetic fields, Astronomy Letters, 42, 339-345, 1601.04855

Kitchatinov, L. L., Rüdiger, G., 1999, Differential rotation models for late-type dwarfs and giants, A\&A, 344, 911-917 
Kitchatinov, L. L., Rüdiger, G., 2005, Differential rotation and meridional flow in the solar convection zone and beneath, Astronomische Nachrichten, 326, 379-385, astro-ph/ 0506239

Komm, R., Howe, R., González Hernández, I., Hill, F., 2014, Solar-Cycle Variation of Subsurface Zonal Flow, Sol. Phys., 289, 3435-3455

Kosovichev, A. G., Schou, J., Scherrer, P. H., Bogart, R. S., Bush, R. I., Hoeksema, J. T., Aloise, J., Bacon, L., Burnette, A., de Forest, C., Giles, P. M., Leibrand, K., Nigam, R., Rubin, M., Scott, K., Williams, S. D., Basu, S., Christensen-Dalsgaard, J., Dappen, W., Rhodes, Jr., E. J., Duvall, Jr., T. L., Howe, R., Thompson, M. J., Gough, D. O., Sekii, T., Toomre, J., Tarbell, T. D., Title, A. M., Mathur, D., Morrison, M., Saba, J. L. R., Wolfson, C. J., Zayer, I., Milford, P. N., 1997, Structure and Rotation of the Solar Interior: Initial Results from the MDI Medium-L Program, Sol. Phys., 170, 43-61

Küker, M., Arlt, R., Rüdiger, G., 1999, The Maunder minimum as due to magnetic Lambda -quenching, A\&A, 343, 977-982

Larson, T., Schou, J., 2009, Variations in Global Mode Analysis, in Solar-Stellar Dynamos as Revealed by Helio- and Asteroseismology: GONG 2008/SOHO 21, (Eds.) M. Dikpati, T. Arentoft, I. González Hernández, C. Lindsey, F. Hill, vol. 416 of Astronomical Society of the Pacific Conference Series, p. 311

Larson, T. P., Schou, J., 2015, Improved Helioseismic Analysis of Medium- $\ell$ Data from the Michelson Doppler Imager, Sol. Phys., 290, 3221-3256, 1511.05217

Larson, T. P., Schou, J., 2018, Global-mode analysis of full-disk data from the michelson doppler imager and the helioseismic and magnetic imager, Solar Physics, 293, 29

Leighton, R. B., Noyes, R. W., Simon, G. W., 1962, Velocity Fields in the Solar Atmosphere. I. Preliminary Report., ApJ, 135, 474

Livingston, W. C., 1969, Solar rotation, 1966 68, Sol. Phys., 7, 144-146

Löptien, B., Birch, A. C., Gizon, L., Schou, J., Appourchaux, T., Blanco Rodríguez, J., Cally, P. S., Dominguez-Tagle, C., Gandorfer, A., Hill, F., Hirzberger, J., Scherrer, P. H., Solanki, S. K., 2015, Helioseismology with Solar Orbiter, Space Sci. Rev., 196, 251-283, 1406.5435

Lynden-Bell, D., Ostriker, J. P., 1967, On the stability of differentially rotating bodies, MNRAS, 136, 293

Mason, J., Hughes, D. W., Tobias, S. M., 2002, The Competition in the Solar Dynamo between Surface and Deep-seated $\alpha$-Effects, ApJ, 580, L89-L92

Miesch, M. S., Hindman, B. W., 2011, Gyroscopic Pumping in the Solar Near-surface Shear Layer, ApJ, 743, 79, 1106.4107

Müller, D., Marsden, R. G., St. Cyr, O. C., Gilbert, H. R., 2013, Solar Orbiter . Exploring the Sun-Heliosphere Connection, Sol. Phys., 285, 25-70, 1207.4579 
Parker, E. N., 1955, Hydromagnetic Dynamo Models., ApJ, 122, 293

Pijpers, F. P., 1997, Solar rotation inversions and the relationship between a-coefficients and mode splittings., A\&A, 326, 1235-1240, astro-ph/9705234

Plaskett, H. H., 1959, Motions in the Sun at the photospheric level, VIII. Solar rotation and photospheric circulation, MNRAS, 119, 197

Rhodes, Jr., E. J., Ulrich, R. K., Deubner, F.-L., 1979, A new technique for measuring solar rotation, ApJ, 227, 629-637

Rhodes, Jr., E. J., Cacciani, A., Korzennik, S., Tomczyk, S., Ulrich, R. K., Woodard, M. F., 1990, Depth and latitude dependence of the solar internal angular velocity, ApJ, 351, $687-700$

Rieutord, M., Rincon, F., 2010, The Sun's Supergranulation, Living Reviews in Solar Physics, 7, 2, 1005.5376

Ritzwoller, M. H., Lavely, E. M., 1991, A unified approach to the helioseismic forward and inverse problems of differential rotation, ApJ, 369, 557-566

Rüdiger, G., 1989, Differential rotation and stellar convection. Sun and the solar stars, Berlin: Akademie Verlag, 1989

Scherrer, P. H., Bogart, R. S., Bush, R. I., Hoeksema, J. T., Kosovichev, A. G., Schou, J., Rosenberg, W., Springer, L., Tarbell, T. D., Title, A., Wolfson, C. J., Zayer, I., MDI Engineering Team, 1995, The Solar Oscillations Investigation - Michelson Doppler Imager, Sol. Phys., 162, 129-188

Schou, J., 1992, On the Analysis of Helioseismic Data, Ph.D. thesis, , Aarhus University, Aarhus, Denmark, (1992)

Schou, J., 1999, Migration of Zonal Flows Detected Using Michelson Doppler Imager F-Mode Frequency Splittings, ApJ, 523, L181-L184

Schou, J., 2003, Wavelike Properties of Solar Supergranulation Detected in Doppler Shift Data, ApJ, 596, L259-L262

Schou, J., Brown, T. M., 1994, Generation of artificial helioseismic time-series., A\&AS, 107

Schou, J., Christensen-Dalsgaard, J., Thompson, M. J., 1994, On comparing helioseismic two-dimensional inversion methods, ApJ, 433, 389-416

Schou, J., Antia, H. M., Basu, S., Bogart, R. S., Bush, R. I., Chitre, S. M., ChristensenDalsgaard, J., Di Mauro, M. P., Dziembowski, W. A., Eff-Darwich, A., Gough, D. O., Haber, D. A., Hoeksema, J. T., Howe, R., Korzennik, S. G., Kosovichev, A. G., Larsen, R. M., Pijpers, F. P., Scherrer, P. H., Sekii, T., Tarbell, T. D., Title, A. M., Thompson, M. J., Toomre, J., 1998, Helioseismic Studies of Differential Rotation in the Solar Envelope by the Solar Oscillations Investigation Using the Michelson Doppler Imager, ApJ, 505, 390-417 
Schou, J., Howe, R., Basu, S., Christensen-Dalsgaard, J., Corbard, T., Hill, F., Komm, R., Larsen, R. M., Rabello-Soares, M. C., Thompson, M. J., 2002, A Comparison of Solar p-Mode Parameters from the Michelson Doppler Imager and the Global Oscillation Network Group: Splitting Coefficients and Rotation Inversions, ApJ, 567, 1234-1249

Schou, J., Scherrer, P. H., Bush, R. I., Wachter, R., Couvidat, S., Rabello-Soares, M. C., Bogart, R. S., Hoeksema, J. T., Liu, Y., Duvall, T. L., Akin, D. J., Allard, B. A., Miles, J. W., Rairden, R., Shine, R. A., Tarbell, T. D., Title, A. M., Wolfson, C. J., Elmore, D. F., Norton, A. A., Tomczyk, S., 2012, Design and Ground Calibration of the Helioseismic and Magnetic Imager (HMI) Instrument on the Solar Dynamics Observatory (SDO), Sol. Phys., 275, 229-259

Schwabe, H., 1844, Sonnen-beobachtungen im jahre 1843, Astron. Nachr., 21, 233-236

Spiegel, E. A., Zahn, J.-P., 1992, The solar tachocline, A\&A, 265, 106-114

Stix, M., 2004, The sun : an introduction

Tassoul, J. L., Hadrava, P., 1983, Book-Review - Theory of Rotating Stars, Bulletin of the Astronomical Institutes of Czechoslovakia, 34, 252

Thompson, M. J., Toomre, J., Anderson, E. R., Antia, H. M., Berthomieu, G., Burtonclay, D., Chitre, S. M., Christensen-Dalsgaard, J., Corbard, T., De Rosa, M., Genovese, C. R., Gough, D. O., Haber, D. A., Harvey, J. W., Hill, F., Howe, R., Korzennik, S. G., Kosovichev, A. G., Leibacher, J. W., Pijpers, F. P., Provost, J., Rhodes, Jr., E. J., Schou, J., Sekii, T., Stark, P. B., Wilson, P. R., 1996, Differential Rotation and Dynamics of the Solar Interior, Science, 272, 1300-1305

Toomre, J., Christensen-Dalsgaard, J., Howe, R., Larsen, R. M., Schou, J., Thompson, M. J., 2000, Time Variability of Rotation in Solar Convection Zone From soi-mdi, Sol. Phys., 192, 437-448

Ulrich, R. K., 1970, The Five-Minute Oscillations on the Solar Surface, ApJ, 162, 993

Vorontsov, S. V., Christensen-Dalsgaard, J., Schou, J., Strakhov, V. N., Thompson, M. J., 2002, Helioseismic Measurement of Solar Torsional Oscillations, Science, 296, 101-103

Warnecke, J., Käpylä, P. J., Mantere, M. J., Brandenburg, A., 2013, Spoke-like Differential Rotation in a Convective Dynamo with a Coronal Envelope, ApJ, 778, 141, 1301.2248

Wilson, P. R., 1992, Helioseismology data and the solar dynamo, ApJ, 399, 294-299

Woodard, M. F., Libbrecht, K. G., 1993, Observations of time variation in the sun's rotation, Science, 260, 1778-1781

Yoshimura, H., 1975, Solar-cycle dynamo wave propagation

Zaatri, A., Corbard, T., 2009, The Solar Subsurface Radial Gradient of Angular Velocity as Revealed by Ring Diagram Analysis, in Solar-Stellar Dynamos as Revealed by Helio- and Asteroseismology: GONG 2008/SOHO 21, (Eds.) M. Dikpati, T. Arentoft, I. González Hernández, C. Lindsey, F. Hill, vol. 416 of Astronomical Society of the Pacific Conference Series, p. 99 


\section{Publications}

\section{Refereed publications}

- A. Barekat, J. Schou, L. Gizon: "The radial gradient of the near-surface shear layer of the Sun", Astronomy \& Astrophysics 570, L12, 2014

- A. Barekat, J. Schou, L. Gizon: "Solar-cycle variation of the rotational shear near the solar surface", Astronomy \& Astrophysics 595, A8, 2016

\section{Conference Contributions}

- HELAS VI/SOHO 28/SPACEINN meeting, Göttingen, Germany, Sept. 1-5, 2014 Talk: Radial gradient of the near-surface shear layer of the Sun

- 15th MHD Days 2014, AIP-Potsdam, Germany, Dec. 2-3, 2015. Talk: Radial gradient of the near-surface shear layer of the Sun

- Sunspot formation: theory, simulations and observations, Nordita, Stockholm, Sweden, March 9-13,2015

Talk: Radial gradient of the near-surface shear layer of the Sun

- Stellar and Planetary Dynamos, Göttingen, Germany, May 26-29, 2015

Poster: Time variation of the near-surface shear layer of the Sun

- Advances in Seismology: a Dialogue Across Disciplines, TIFR-Mumbi, India, Dec. 7-11 2015

Invited talk: Solar rotation near the surface 



\section{Acknowledgments}

I would like to thank the members of my committee Olga Shishkina, Laurent Gizon, Ulrich Christensen, Stefan Dreizler, Ariane Frey and Ansgar Reiners for accepting to be the examiners of my thesis. I would like to thank the International Max Planck Research Schoul (IMPRS) for funding me during my PhD studies and the school coordinator Sonja Schuh for all her support.

My deepest appreciation goes to my mentor Jesper Schou who helped me to reach to my destination in this challenging journey. I am also thankful to Hannah Schunker, Thomas Duvall and Aaron Birch for always answering any of my scientific questions with utmost patience and respect. My very special thank goes to Robert Cameron, my wonderful office mate, who always engaging in meaningful scientific topics with me including answering any of my questions. I never forget the happy hour watches drawing on my wrist which keeps me going in my frustrating days during my $\mathrm{PhD}$.

\section{I am thankful to}

-my friends Emanuele, Jan, Martin and Björn for being a source of joy and a great support system throughout all my endeavors, thank you very much.

-Astrid and Hardi, for being the greatest friends in my most challenging moments of my journey. I am so grateful to you both.

-all my IMPRS friends who make Göttingen one of the most memorable cities in my life and, of course, "Ich bin Erste" at the coffee time.

-Tim Larson who always provides me any HMI or MDI data I needed for this work.

-my parents, for being the world's greatest parents, thank you and I love you. To my sisters Samira, Leili, and my brother Atta and my sister in law Nahid for your love and undying support. To my German family Gabriela, Reinhard, Lars and Steffi for your love and constant support, thank you very much.

Lastly, I want to thank the love of my life and my husband Jörn whose enduring emotional support and everlasting love got me through each obstacle with outmost confidence and conviction. Thank you for believing in me. I love you now, I love you always.

The MDI data are provided from $\mathrm{SOHO}$ which is a project of international cooperation between ESA and NASA. The HMI data are courtesy of NASA/SDO and the HMI science team. 